\title{
Vapor Pressure Equation for Water in the Range 0 to $100{ }^{\circ} \mathrm{C}$
}

\author{
Arnold Wexler and Lewis Greenspan
}

\author{
Institute for Basic Standards, National Bureau of Standards, Washington, D.C. 20234
}

(February 19, 1971)

\begin{abstract}
Some precise measurements of the vapor pressure of liquid water at seven temperatures in the range 25 to $100{ }^{\circ} \mathrm{C}$ were reported recently by H. F. Stimson of NBS. These measurements have an estimated standard deviation of $20 \mathrm{ppm}$ or less, except at $25{ }^{\circ} \mathrm{C}$ where the estimated standard deviation is $44 \mathrm{ppm}$. We have derived a formula which yields computed values of vapor pressure agreeing with Stimson's measurements to within $7 \mathrm{ppm}$.

We integrated the Clausius-Clapeyron equation using the accurate calorimetric data of Osborne, Stimson, and Ginnings and the Goff and Gratch formulations for the virial coefficients of water vapor to obtain an equation that has a rational basis. This equation was then adjusted to bring it into closer accord with Stimson's pressure measurements. Two tables are given of the vapor pressure, expressed in pascals, as a function of temperature at $0.1-\mathrm{deg}$ intervals over the range 0 to $100{ }^{\circ} \mathrm{C}$, one on the International Practical Temperature Scale of 1948 and the other on the International Practical Temperature Scale of 1968.
\end{abstract}

Key words: Clausius-Clapeyron equation; saturation vapor pressure over water; vapor pressure; vapor pressure of water; water vapor; steam; virial coefficients.

\section{Introduction}

In establishing and maintaining humidity standards, in calibrating hygrometers, and in making precision humidity measurements, an accurate equation for the vapor pressure of water is essential. Such an equation also plays a vital role in steam power technology. It is important in the fields of meteorology and air conditioning where precise calculations relating to the water vapor content of atmospheric air are often made. It is employed in chemical thermodys namics, where the vapor pressure of the pure water substance is used as a reference standard in calibrating boiling point apparatus, in comparative vapor pressure measurements, in ebulliometry and in evaluating vapor pressure data of organic liquids.

It has been the practice at NBS to use the Goff and Gratch vapor pressure formulation $[1]^{1}$ in all work pertaining to hygrometry. Recently, H. F. Stimson [2] published the results of some precise measurements of the vapor pressure of water at seven temperatures from 25 to $100{ }^{\circ} \mathrm{C}$. These vapor pressures, obtained with the steam boiler and precision manometer used at NBS in the 1940's to calibrate standard platinum resistance thermometers [3], have an estimated standard deviation of $20 \mathrm{ppm}$ or less except at $25^{\circ} \mathrm{C}$ where the estimated standard deviation is $44 \mathrm{ppm}$. Although vapor pressures calculated using the Goff and Gratch formulation are in reasonably good accord with Stim- son's measurements, differing at most, at $25{ }^{\circ} \mathrm{C}$, by $95 \mathrm{ppm}$, we concluded that it would be desirable to have a formulation that was in closer agreement with Stimson's measurements, preferably within the estimated uncertainty of the latter. The purpose of this paper is to present such a formulation. We shall limit our considerations to temperatures 0 to $100{ }^{\circ} \mathrm{C}$.

\section{Derivation}

We start with a modified version of the ClausiusClapeyron equation [4]

$$
\frac{d p}{d T}=\frac{\gamma}{T v}
$$

where $p$ is the pressure of the saturated vapor, $v$ is the specific volume of the saturated vapor, $T$ is the absolute thermodynamic temperature, $\gamma$ is an experimentally measured calorimetric quantity, and $d p / d T$ is the derivative of the vapor pressure with respect to the absolute thermodynamic temperature. The quantity $\gamma[4]$ is the heat supplied to water to evaporate a unit mass of water isothermally and withdraw its vapor from the calorimeter. It differs from the latent heat of vaporization, $L$, by a small quantity, $\beta$, that is,

$$
\gamma=L+\beta
$$

where

$$
\beta=\frac{v^{\prime}}{v-v^{\prime}} L
$$


and $v^{\prime}$ is the specific volume of the saturated liquid. The quantity $\beta$ may be pictured as the heat necessary to vaporize water to fill the space no longer filled with liquid in the $\gamma$ experiment.

The virial equation of state for water vapor, expressed as a power series in $p$,

$$
p v=R T\left[1+B^{\prime} p+C^{\prime} p^{2}+\ldots .\right]
$$

when substituted into eq (1) yields

$$
\frac{d p}{p}=\frac{\gamma}{R T^{2} Z} d T
$$

where

$$
Z=1+B^{\prime} p+C^{\prime} p^{2}+\ldots
$$

$Z$ is the compressibility factor, $R$ is the gas constant for water vapor, $B^{\prime}$ is the second pressure-series virial coefficient, and $C^{\prime}$ is the third pressure-series virial coefficient. For temperatures up to $100{ }^{\circ} \mathrm{C}$, fourth and higher virial coefficients affect $Z$ by less than $12 \mathrm{ppm}$ and therefore will be neglected.

Performing some simple mathematical manipulations and integrations, eq (5) becomes

$$
\int_{p_{0}}^{p} d(\ln p)=\int_{T_{0}}^{T} \frac{\gamma}{R T^{2}} d T-\int_{T_{0}}^{T} \frac{\gamma}{R T^{2}}\left(\frac{Z-1}{Z}\right) d T
$$

where $p_{0}$ and $p$ correspond to vapor pressures at temperatures $T_{0}$ and $T$, respectively. The first integral on the right-hand side of the equation provides the major contribution to the vapor pressure. The second integral on the right-hand side of the equation accounts for the deviation of water vapor from ideal gas behavior.

We shall show later that the quantity $\gamma$ can be represented with high precision by the polynomial equation

$$
\gamma=a+b T+c T^{2}+d T^{3}
$$

which, when substituted into the first integral on the right-hand side of eq (7) yields

$$
\begin{gathered}
\int_{p_{0}}^{p} d(\ln p)=\frac{1}{R}\left[-a\left(\frac{1}{T}-\frac{1}{T_{0}}\right)+b \ln \frac{T}{T_{0}}+c\left(T-T_{0}\right)\right. \\
\left.+\frac{d}{2}\left(T^{2}-T_{0}^{2}\right)\right]-\frac{1}{R} \int_{T_{0}}^{T} \frac{\gamma}{T^{2}}\left(\frac{Z-1}{Z}\right) d T
\end{gathered}
$$

Equation (9) reduces to

$$
\begin{aligned}
\int_{p_{0}}^{p} d(\ln p)=-\frac{a}{R T} & +\frac{b}{R} \ln T+\frac{c}{R} T+\frac{d}{2 R} T^{2} \\
& +e-\frac{1}{R} \int_{T_{0}}^{T} \frac{\gamma}{T^{2}}\left(\frac{Z-1}{Z}\right) d T
\end{aligned}
$$

where

$$
e=+\frac{a}{R T_{0}}-\frac{b}{R} \ln T_{0}-\frac{c}{R} T_{0}-\frac{d}{2 R} T_{0}^{2}
$$

The derivation of eq (10) is similar in some respects to that of Goff and Gratch $[5,6]$.

In 1939, Osborne, Stimson and Ginnings [7] reported weighted mean values of $\gamma$ from 0 to $200{ }^{\circ} \mathrm{C}$ based on precise calorimetric measurements in the range 0 to $100{ }^{\circ} \mathrm{C}$ and on similar measurements made in the same laboratory during 1930-32 in the range 50 to $270{ }^{\circ} \mathrm{C}$ [8] and in 1937 in the range 100 to $374{ }^{\circ} \mathrm{C}$ [9]. We fitte! eq (8) to these weighted means by the method of least squares, retaining the units international joules per gram for $\gamma$ and temperature $t$ in degrees $\mathrm{C}$ based on the International Temperature Scale of 1927 (ITS-27) but letting the absolute temperature $T_{48}=t+273.15$. In our range of interest, 0 to $100{ }^{\circ} \mathrm{C}, t$ has the same numerical value on the International Temperature Scale of 1927 (ITS-27) [10], the International Temperature Scale of 1948 (ITS-48) [11], and the International Practical Temperature Scale of 1948 (IPTS-48) [12]. Therefore, we will use $t$ interchangeably on these scales as may prove convenient. However, the absolute temperature $T_{48}$, due to the choice of 273.15 conforms to IPTS-48. The coefficients of eq (8) have the following numerical values: $\quad a=3.4660697 \times 10^{3}, \quad b=-5.6067899$, $c=1.0963233 \times 10^{-2}$, and $d=-1.2366148 \times 10^{-5}$. The residual standard deviation ${ }^{2}$ is 0.231 international joules per gram, about 1 part in 10,000.

Virial coefficients for steam are usually derived from $p-v$ - $t$ measurements $[13,14,15,16]$. Unfortunately, there are no $p-v-t$ measurements for steam (water vapor) in the range 0 to $100{ }^{\circ} \mathrm{C}$.

The early experiments of Knoblauch, Linde and Klebe [17], which were performed at temperatures from 100 to $180{ }^{\circ} \mathrm{C}$, are suspect because of the static method used which leads to systematic errors due to the adsorption of water vapor on the walls of the container [13]. The experiments of Keyes, Smith, and Gerry [13], made by a continuous flow-method, cover the range 195 to $460{ }^{\circ} \mathrm{C}$. These, too, are suspect below the critical temperature because of possible adsorption effects [16]. Other investigators have provided data at higher temperatures and pressures. It is possible to extrapolate the virial coefficients obtained at higher temperatures to lower temperatures [13, 14], but such extrapolations do not give values with the requisite accuracy for our purpose. Alternately, calorimetric data can be employed to obtain enthalpy coefficients, which, when integrated with respect to temperature, yield virial coefficients. This procedure was employed by Goff and Gratch $[5,6]$, who used the 38 to $125^{\circ} \mathrm{C}$ throttling experiment data of Collins and Keyes [18] and the $\gamma$ data of Osborne, Stimson and Ginnings [7] to derive second and third pressureseries virial coefficients valid for the range 0 to 100

${ }^{2}$ The residual standard deviation is given by

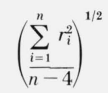

where $r_{i}$ is the difference between the weighted mean and the corresponding computed value, $n$ is the number of fitted points, $n-4$ is the number of degrees of freedom and 4 is the number of coefficients in eq (8). 
${ }^{\circ} \mathrm{C}[5,6,19]$. Because the Goff and Gratch empirical relationships for the pressure-series virial coefficients are based on experimental data in the range of temperatures of interest to us, and because, of the various virial coefficients we tried, the Goff and Gratch virial coefficients yielded values of vapor pressure in closest agreement with Stimson's experimental values, we chose to use them. We converted the Goff and Gratch relationships [19] to SI units compatible with eqs (4) and (6) to obtain

$$
B^{\prime}=\left[+\frac{0.4086}{T_{48}}-\left(\frac{665.19}{T_{48}^{2}}\right) \times 10^{\frac{72000}{T_{48}^{2}}}\right] \times 10^{-5}
$$

and

$$
\begin{aligned}
C^{\prime}=2.374\left[+\frac{0.4086}{T_{48}}-\left(\frac{665.19}{T_{48}^{2}}\right)\right. & \\
& \left.\times 10^{\frac{72000}{T_{48}^{2}}}\right]^{3} \times 10^{-8}
\end{aligned}
$$

where $B^{\prime}$ is in units of reciprocal pressure, $1 /$ pascal, ${ }^{3}$ $C^{\prime}$ is in units of the square of the reciprocal pressure, (1/pascal) $)^{2}$, and $T_{48}$ is based on IPTS -48 . The equations as derived by Goff and Gratch presumably were in terms of absolute temperature on ITS-27. Over the range 0 to $100{ }^{\circ} \mathrm{C}$, the errors arising in $B^{\prime}, C^{\prime}, Z$, and $Z-1$ from using $T_{48}$ without conversion are negligible.

The Clausius-Clapeyron equation is an exact thermodynamic expression in which $T$ is the absolute thermodynamic temperature. It therefore follows that the temperature $T$ in eq (10), which was derived from the Clausius-Clapeyron equation, is also the absolute thermodynamic temperature. We will assume that $T_{48}$ is a reasonably close approximation to the absolute thermodynamic temperature $T$ and reserve for later discussion the reasons for this assumption. We inserted into eq (10) the coefficients of eq (8) on the basis of $T_{48}$, and the virial coefficients given by eqs (12) and (13) as functions of $T_{48}$. We selected as lower limits of integration for substitution into eqs (10) and (11) the pressure $p_{0}=101325.0$ pascals, which is one standard atmosphere, and the temperature $T_{0}=373.15$ $\mathrm{K}$, which is the absolute temperature assigned to the steam point at one standard atmosphere on $T_{48}$. The gas constant for water vapor, $R$, is 0.46151 joules per gram kelvin and was derived from the NAS-NRC recommended value [20] of 8.3143 joules per mol kelvin for the universal gas constant and 18.01534 grams for the molar mass of water vapor on the unified carbon-12 scale. The units of the quantity $\gamma$ were made consistent with $R$ by the conversion factor 1 international joule $=1.000165$ (absolute) joule [21] After inserting the appropriate constants and conversion factor, eq (10) becomes

$$
\begin{aligned}
& \int_{p_{0}}^{p} d(\ln p)=\sum_{i=0}^{3} A_{i} T_{48}^{i-1}+B \ln T_{48} \\
&+C \int_{373.15}^{T_{48}} \frac{\gamma}{T_{48}^{2}}\left(\frac{Z-1}{Z}\right) d T
\end{aligned}
$$

\footnotetext{
${ }^{3} 1$ pascal $=1$ newton $/ \mathrm{m}^{2}=10^{-5} \mathrm{bar}=10^{-2} \mathrm{mb}=7.50062 \times 10^{-3} \mathrm{~mm} \mathrm{Hg}$.
}

where $A_{0}=-7.51152 \times 10^{3}, \quad A_{1}=85.0869143, \quad A_{2}$ $=2.3759056 \times 10^{-2}, \quad A_{3}=-1.399697 \times 10^{-5}, \quad B=$ $-12.150799, C=-2.1671578, \quad p$ is expressed in pascals, $T_{48}=t_{48}+273.15$ in kelvins, and $t_{48}$ is in degrees Celsius on IPTS-48.

Because eq (14) is implicit in $p$ we calculated $p$ by iteration, numerically evaluating the integral at 1/4-deg intervals by means of the trapezoidal rule [22]. Iteration at each interval was terminated when successive values of $p$ differed by less than one $\mathrm{ppm}$. Evaluating the integral at 1/4-deg intervals was considered satisfactory. A check calculation at $80{ }^{\circ} \mathrm{C}$ showed that decreasing the interval to $0.025 \mathrm{deg}$ produced a change in $p$ of much less than one ppm from that obtained using $1 / 4$-deg intervals.

The calculation of $p$ by eq (14) is best accomplished with the aid of a high-speed digital computer. For desk-type calculations, an explicit equation without the integral is desirable. Equation (14) was converted into such an explicit form. A polynomial of the fourth degree was fitted, by the method of least squares, to numerical values of the integral at 1 -deg intervals from 0 to $100^{\circ} \mathrm{C}$ to give

$$
C \int_{373.15}^{T_{48}} \frac{\gamma}{T_{48}^{2}}\left(\frac{Z-1}{Z}\right) d T=\sum_{i=0}^{4} D_{i} T_{48}^{i}
$$

where $D_{0}=-5.2075843 \times 10^{-2}, D_{1}=-5.769527 \times 10^{-5}$, $D_{2}=3.2537152 \times 10^{-6}, \quad D_{3}=-1.6189993 \times 10^{-8}, \quad$ and $D_{4}=2.381486 \times 10^{-11}$. Substituting eq (15) into eq (14), integrating the left-hand side of eq (14), and combining terms yielded

$$
\ln p=\sum_{i=0}^{5} E_{i} T_{48}^{i-1}+B \ln T_{48} .
$$

The coefficients are listed in table 1.

On plotting the very small differences between $\ln p$ given by eq (16) and Stimson's measurements as a function of temperature, we observed that the course of the curve was smooth. A polynomial of the fourth degree was fitted to this difference curve. By adding the corresponding coefficients of like powers in $T_{48}$ to eq (16) we obtained

$$
\ln p=\sum_{i=0}^{5} E_{i} T_{48}^{i-1}+B \ln T_{48}
$$

which is identical to eq (16), except that the numerical values of the coefficients $E_{1}$ through $E_{5}$ are slightly different. The coefficients are listed in table 1.

We investigated the feasibility of simplifying eq (17). Numerous formulas have been proposed for empirically representing the functional relationship between vapor pressure and temperature $[23,24,25,26]$. A procedure often followed is to select the formula with the least number of terms that not only best fits the vapor pressure data but also yields derivatives that are smooth and regular. We chose the equation

$$
\ln p=\sum_{i=0}^{n} E_{i} T_{48}^{i-1}+B \ln T_{48}
$$


TABLE 1. Coefficients to vapor pressure equations on IPTS-48

\begin{tabular}{|c|c|c|c|c|c|}
\hline & $\begin{array}{c}\mathrm{Eq}(16) \\
n=5\end{array}$ & $\begin{array}{c}\mathrm{Eq}(17) \\
n=5\end{array}$ & $\begin{array}{c}\mathrm{Eq}(18 \mathrm{a}) \\
n=2\end{array}$ & $\begin{array}{c}\mathrm{Eq}(18 \mathrm{~b}) \\
n=3\end{array}$ & $\begin{array}{c}\mathrm{Eq}(18 \mathrm{c}) \\
n=4\end{array}$ \\
\hline$E_{0}$ & $-7.51152 \times 10^{3}$ & $-7.51152 \times 10^{3}$ & $-7.2465822 \times 10^{3}$ & $-6.7777203 \times 10^{3}$ & $-7.7847207 \times 10^{3}$ \\
\hline$E_{1}$ & $9.65609269 \times 10^{1}$ & $9.65389644 \times 10^{1}$ & $7.7641232 \times 10^{1}$ & $5.4409359 \times 10^{1}$ & $1.1670432 \times 10^{2}$ \\
\hline$E_{2}$ & $2.3701361 \times 10^{-2}$ & $2.3998970 \times 10^{-2}$ & $5.7447142 \times 10^{-3}$ & $-8.0404143 \times 10^{-3}$ & $5.1177435 \times 10^{-2}$ \\
\hline$E_{3}$ & $-1.0145982 \times 10^{-5}$ & $-1.1654551 \times 10^{-5}$ & & $7.1544503 \times 10^{-6}$ & $-5.438695 \times 10^{-5}$ \\
\hline$E_{4}$ & $-1.6189993 \times 10^{-8}$ & $-1.2810336 \times 10^{-8}$ & & & $3.189024 \times 10^{-8}$ \\
\hline$E_{5}$ & $2.3816486 \times 10^{-11}$ & $2.0998405 \times 10^{-11}$ & & & \\
\hline$B$ & $-1.2150799 \times 10^{1}$ & $-1.2150799 \times 10^{1}$ & -8.2470402 & -3.8358214 & $-1.6463576 \times 10^{1}$ \\
\hline
\end{tabular}

because it is analogous in form to our derived eq (17) and fitted it for $2 \equiv n \equiv 4$ by the method of least squares to values of vapor pressure generated at 1-deg intervals from 0 to $100{ }^{\circ} \mathrm{C}$ by eq (17). The coefficients are listed in table 1. For convenience in identifying the several versions of eq (18), each has been designated with a letter $a$ through $c$ corresponding to $n=2$, 3 , and 4 , respectively.

\section{Results}

The measurements made at the PhysikalischTechnische Reichsanstalt by Holborn and Henning [27] in 1908 from 50 to $200{ }^{\circ} \mathrm{C}$ and by Scheel and Heuse [28] in 1910 from 0 to $50{ }^{\circ} \mathrm{C}$ have, until now, constituted the major sources of data for the vapor pressure of water in the 0 to $100{ }^{\circ} \mathrm{C}$ range. In 1919, PTR published revised values of the vapor pressure [29] and these have served as the input data for many empirical formulations [30, 31].

The steam point has been used as one of the defining fixed points for the several successive practical temperatures scales that have been adopted internationally $[10,11,12,32]$. Because of this, the vapor pressure near the steam point has been investigated in great detail [33, 34, 35, 36, 37, 38]. Most of these measurements were made over narrow temperature spans around $100{ }^{\circ} \mathrm{C}$. The largest temperature range, 73 to $130{ }^{\circ} \mathrm{C}$, was covered in 1939 by Moser and Zmaczynski [37] who used both a static and a dynamic method in their determinations. Beattie and Blaisdell in 1937 [36] and Michels, Blaisse, Ten Seldam and Wouters in 1943 [38] limited their observations to several degrees below and above $100{ }^{\circ} \mathrm{C}$.

Douslin and McCullough in 1963 [39] and Douslin in 1970 [40], using an inclined dead-weight piston gage, made vapor pressure measurements from 0 to $20^{\circ} \mathrm{C}$.

The above series of measurements, plus the measurements of Stimson, comprise the most important determinations covering the 0 to $100{ }^{\circ} \mathrm{C}$ span.

We compared our formulation with these experimental data and with other formulations in order to obtain a general perspective on the degree of accord achieved. However, because of the high precision and internal consistency of the Stimson measurements we judged the efficacy of our vapor pressure equations primarily on their agreement with the Stimson data.

\subsection{Comparisons With Stimson's Measurements}

Differences in vapor pressure between Stimson's measurements and eqs (14), (16), (17), and (18) are given in table 2. The standard deviation of Stimson's values are also shown. Equation (14) yields values which agree with Stimson's measurements to within one standard deviation of the latter, except at 50 and $80{ }^{\circ} \mathrm{C}$ where the agreement is within two standard deviations. The maximum difference is $32 \mathrm{ppm}$ and occurs at $25{ }^{\circ} \mathrm{C}$. The conversion of eq (14), an implicit equation, to eq (16), an explicit equation, has little effect on the differences. Equation (17), which was

TABLE 2. Comparison between calculated vapor pressures and Stimson's measurements on IPTS-48

\begin{tabular}{|c|c|c|c|c|c|c|c|c|c|c|c|c|c|c|c|}
\hline \multirow{3}{*}{$\begin{array}{c}\text { Temperature } \\
\text { IPTS-48 } \\
{ }^{\circ} \mathrm{C}\end{array}$} & \multicolumn{3}{|c|}{ Stimson's measurements } & \multirow{2}{*}{\multicolumn{2}{|c|}{$\mathrm{Eq}(14)$}} & \multirow{2}{*}{\multicolumn{2}{|c|}{$\begin{array}{c}\mathrm{Eq}(16) \\
n=5\end{array}$}} & \multirow{2}{*}{\multicolumn{2}{|c|}{$\begin{array}{c}\mathrm{Eq}(17) \\
n=5\end{array}$}} & \multirow{2}{*}{\multicolumn{2}{|c|}{$\begin{array}{c}\mathrm{Eq}(18 \mathrm{a}) \\
n=2\end{array}$}} & \multirow{2}{*}{\multicolumn{2}{|c|}{$\begin{array}{c}\mathrm{Eq}(18 \mathrm{~b}) \\
n=3\end{array}$}} & \multirow{2}{*}{\multicolumn{2}{|c|}{$\begin{array}{c}\mathrm{Eq}(18 \mathrm{c}) \\
n=4\end{array}$}} \\
\hline & \multirow{2}{*}{$\begin{array}{c}\begin{array}{c}\text { Vapor } \\
\text { pressure }\end{array} \\
\text { pascals }\end{array}$} & \multicolumn{2}{|c|}{$\begin{array}{l}\text { Standard } \\
\text { deviation }\end{array}$} & & & & & & & & & & & & \\
\hline & & pascals & ppm & pascal & ppm & pascal & ppm & pascal & ppm & pascal & ppm & pascal & ppm & pascal & ppm \\
\hline 25 & 3167.0 & 0.14 & 44 & +0.1 & +32 & +0.1 & +32 & 0.0 & 0 & 0.0 & 0 & 0.0 & 0 & 0.0 & 0 \\
\hline 40 & 7377.27 & .15 & 20 & +.11 & +15 & +.08 & +11 & -.05 & -7 & -.14 & -19 & -.02 & -3 & -.07 & -9 \\
\hline 50 & 12338.30 & .18 & 15 & +.22 & +18 & +.18 & +14 & +.09 & +7 & -.15 & -12 & +.10 & +8 & +.05 & +4 \\
\hline 60 & 19924.22 & .16 & 8 & -.15 & -8 & -.17 & - & -.12 & -6 & -.33 & -17 & -.13 & -7 & -.18 & -9 \\
\hline 70 & 31166.15 & .22 & 7 & -.20 & -6 & -.20 & - & +.15 & +5 & +.40 & +12 & +.16 & +5 & $\begin{array}{r}.05 \\
+\quad .05\end{array}$ & +2 \\
\hline 80 & 47363.9 & .52 & 11 & -.7 & -15 & -.9 & -19 & -.2 & -4 & +.9 & +19 & 0.0 & 0 & -.4 & -8 \\
\hline 100 & 101325.0 & 1.59 & 16 & +.1 & +1 & +.2 & +2 & 0.0 & 0 & -5.3 & -52 & -.5 & -5 & -.4 & -4 \\
\hline
\end{tabular}


obtained by making a minor adjustment to the coefficients of eq (16), is in closer accord with Stimson's measurements. The maximum difference is $7 \mathrm{ppm}$. Of the three variants of eq (18), which are least square fits to values generated by eq (17), eqs (18b) and (18c) are in excellent agreement with Stimson's measurements, differing at most by $9 \mathrm{ppm}$. The differences for eq (18a) are larger, becoming as great as $52 \mathrm{ppm}$ at $100{ }^{\circ} \mathrm{C}$. There does not appear to be any reason for choosing any one equation from eqs (17), (18b), and (18c) on the basis of the difference between calculated vapor pressures and Stimson's measurements.

Using eq (17) as a base line, differences between eqs (18a) through (18c) and eq (17) were computed and plotted in pascals on figure 1 and in ppm on figure 2. The differences between Stimson's measurements and eq (17) are also shown. As $n$ decreases from 4 to 2 the differences increase. Equation (18c) deviates systematically from eq (17) by $3 \mathrm{ppm}$. The differences between eq (18b) and (17) and eqs (18a) and (17) show oscillatory patterns; they do not exceed $9 \mathrm{ppm}$ for eq (18b) but reach 52 ppm for (18a). Equations (18c) and (18b) offer a reduction in terms without a significant loss in accuracy. If a lower order of accuracy is acceptable, then eq (18a) could be used. In our opinion, eq (17) is preferable because it has a thermodynamic origin and, therefore, may inspire more confidence for interpolation and extrapolation. However, eq (17) has seven terms and where a simpler formula is desired, eq (18b), with five terms, would be our choice.

\subsection{Comparisons With Douslin's Measurements}

Douslin $[39,40]$ has made two sets of vapor pressure measurements over liquid water at temperatures below $25^{\circ} \mathrm{C}$ using an inclined-piston dead-weight pressure gage. ${ }^{4}$ The earlier measurements are as much as 4 pascals (30 microns) higher at some temperatures which Douslin attributed to incomplete degassing of his water sample in the earlier work. In the later work, the measurements were made in two series, Series I with the piston unweighted and Series II with the piston weighted. The two series overlap in temperature. We have compared Douslin's later measurements [40] with eq (17). The differences are given in table 3 . Douslin's vapor pressures, measured on IPTS-68, were adjusted to IPTS-48 for this comparison. He reported that his estimated maximum systematic error varied from 0.32 pascal at $0{ }^{\circ} \mathrm{C}$ to 0.81 pascal at $20^{\circ} \mathrm{C}$, or, in relative terms, from 520 to $350 \mathrm{ppm}$. There is a systematic difference between Douslin's measurements and our calculations, his observed values deviating from our computed values by two to three times his estimated maximum systematic error. It must be emphasized that eq (17) was adjusted to fit Stimson's measurements in the range 25 to $100{ }^{\circ} \mathrm{C}$ and that our calculations below $25^{\circ} \mathrm{C}$ are therefore, in part, extrapolations and may not necessarily fit other experimental measurements with comparable accuracy.

${ }^{4}$ We wish to thank Dr. D. R. Douslin for permission to quote from his second paper prior to publication.

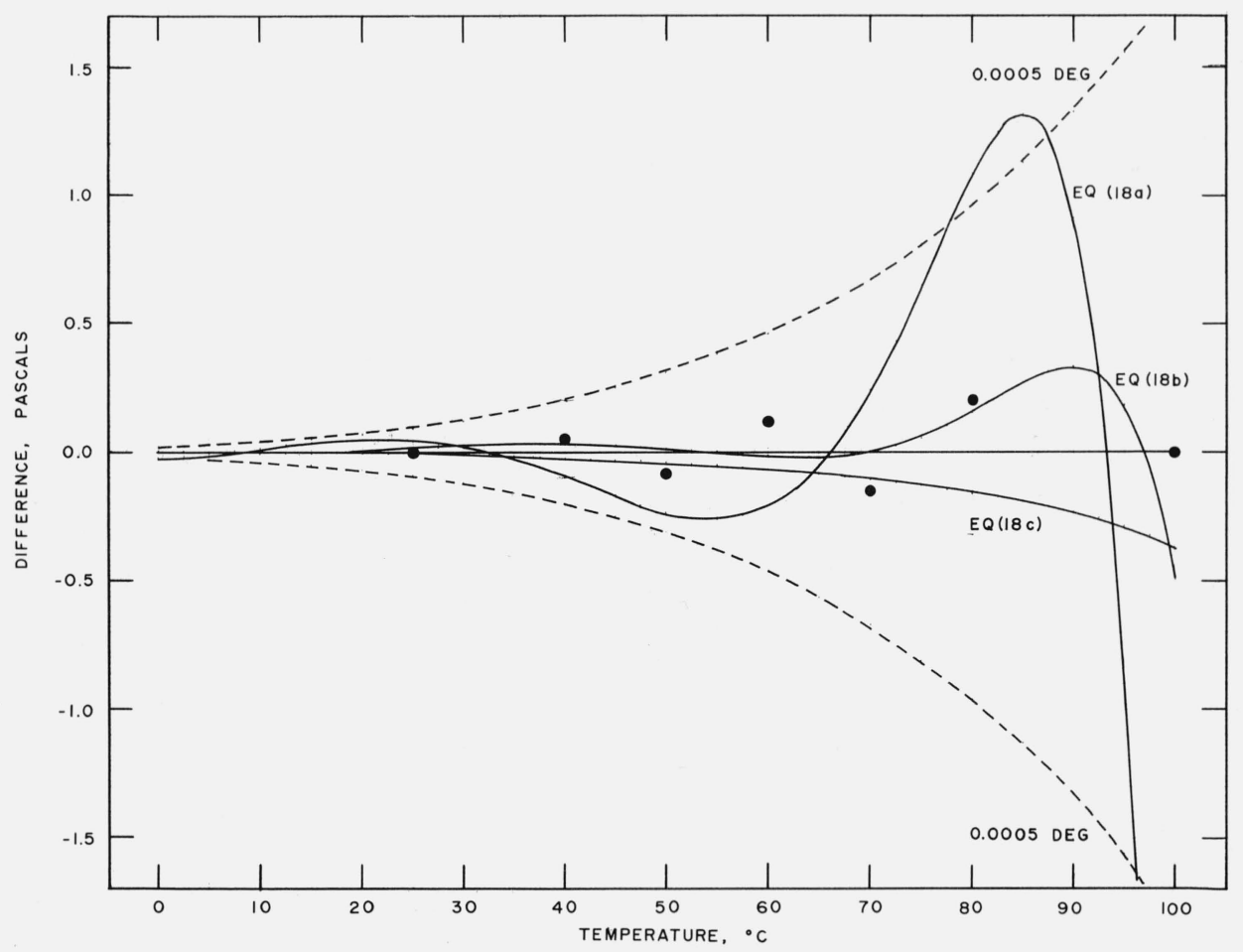

Figure 1. Comparison of equations.

Vapor pressure difference [other-eq (17)] in pascals between eqs (18a), (18b), and (18c) and eq (17). Vapor pressure difference between Stimson's measurements and eq (17) shown by solid circles. Dashed lines are difference curves for a temperature deviation of \pm 0.0005 deg. 


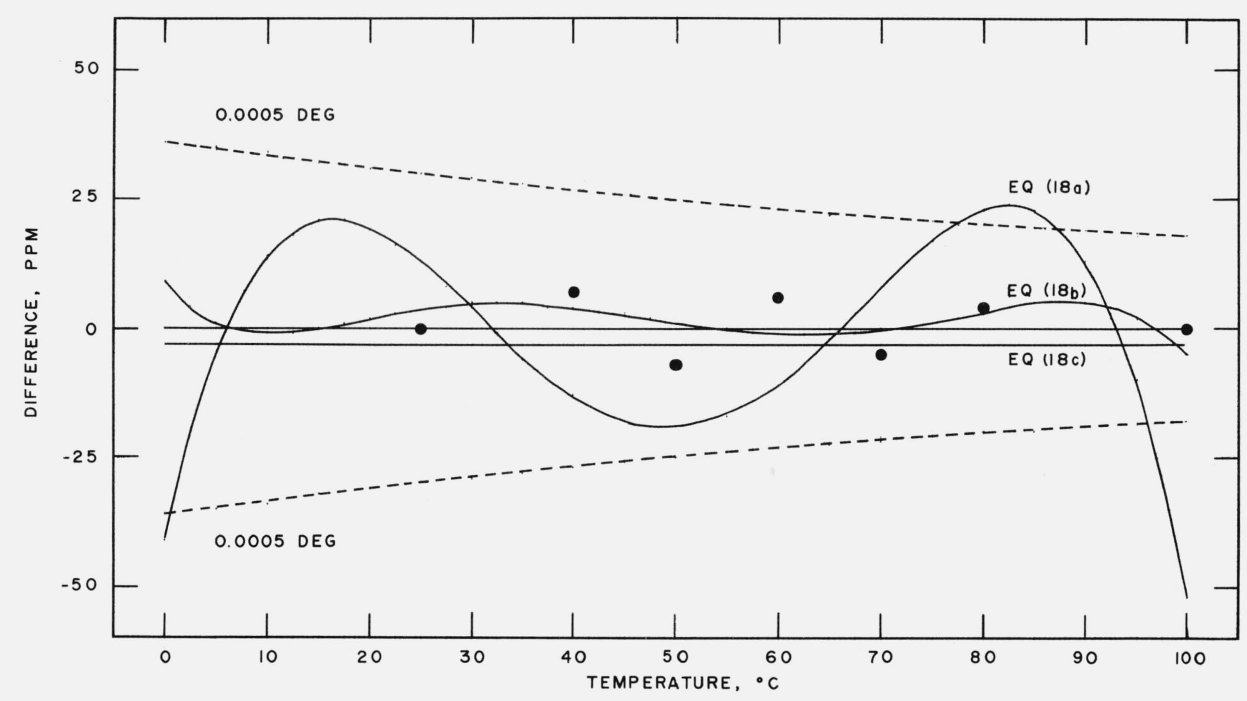

Figure 2. Comparison of equations.

Relative vapor pressure difference $\left[\frac{\text { other-eq }(17)}{\text { eq }(17)} \times 10^{6}\right]$ in ppm between eqs (18a), (18b), and (18c) and eq (17). Relative vapor pressure difference between Stimson's measurements and eq (17) shown by solid circles. Dashed lines are difference curves for a temperature deviation of $\pm 0.0005 \mathrm{deg}$.

TABLE 3. Comparison between calculated vapor pressures and Douslin's measurements on IPTS-48

\begin{tabular}{|c|c|c|c|c|c|}
\hline \multirow{2}{*}{$\begin{array}{l}\text { Tempera- } \\
\text { ture } \\
\text { IPTS-48 }\end{array}$} & \multicolumn{3}{|c|}{$\begin{array}{l}\text { Douslin's Adjusted } \\
\text { Measurements }\end{array}$} & \multirow{2}{*}{\multicolumn{2}{|c|}{$\begin{array}{c}\text { Vapor Pressure } \\
\text { Difference } \\
\text { Douslin-- } \\
\text { Eq (17) }\end{array}$}} \\
\hline & $\begin{array}{c}\text { Vapor } \\
\text { pressure }\end{array}$ & $\begin{array}{r}\text { Maximum } \\
\mathrm{e}\end{array}$ & stematic & & \\
\hline${ }^{\circ} \mathrm{C}$ & pascals & pascals & ppm & pascals & ppm \\
\hline
\end{tabular}

Series I

\begin{tabular}{l|r|r|r|r|r}
\hline & & & & & \\
0.01 & a 611.5 & 0.32 & 520 & +0.7 & +1140 \\
1 & 612.2 & .33 & 540 & +1.0 & +1630 \\
2 & 657.4 & .35 & 530 & +0.8 & +1220 \\
3 & 706.2 & .35 & 490 & +0.7 & +990 \\
4 & 758.6 & .36 & 470 & +1.1 & +1450 \\
5 & 813.7 & .37 & 460 & +0.8 & +980 \\
7.5 & 873.0 & .39 & 440 & +1.1 & +1260 \\
10 & 1037.2 & .43 & 410 & +1.0 & +960 \\
15 & 1228.8 & .45 & 370 & +1.6 & +1300 \\
& 1706.1 & .57 & 340 & +1.7 & +1000 \\
\hline
\end{tabular}

Series II

\begin{tabular}{l|r|r|r|r|r}
\hline 7.5 & 1036.8 & 0.43 & 410 & +0.6 & +580 \\
12.5 & 1448.2 & .61 & 420 & -0.4 & -280 \\
17.5 & 2000.1 & .73 & 370 & +1.1 & +550 \\
20 & 2338.7 & .81 & 350 & +1.4 & +600 \\
\hline
\end{tabular}

${ }^{a}$ Metastable state; vapor pressure with respect to supercooled water.

\subsection{Comparisons at the Ice and Triple Points}

In table 4 we have compared several PhysikalischTechnische Reichsanstalt measurements of vapor pressure at $0{ }^{\circ} \mathrm{C}[28,41,42]$, Prytz's measurement at the triple point [43], and steam table values at both the ice and triple points $[16,44,48]$ with corresponding values calculated with eq (17). It may be noted that the ice and triple points should not be subject to any temperature scale uncertainty as may be the case at other temperatures with data from the early 1900's. To complete the tabulation, Douslin's values at $0^{\circ}$ and $0.01{ }^{\circ} \mathrm{C}$ [40] are repeated here.

At $0{ }^{\circ} \mathrm{C}$, the PTR data are lower and Douslin's measurement is higher than our calculated value. At the triple point, Prytz's and Douslin's measurements are higher than our calculated value. We are in very good agreement with the steam table values.

\subsection{Comparisons at and below the Steam Point}

We have compared the Moser and Zmaczynski data $[37],{ }^{5}$ the Beattie and Blaisdell data [36], and the Michels, Blaisse, Ten Seldam and Wouters data [38] with eq (17). This is shown as a plot of vapor pressure differences, in pascals, in figure 3 and as a plot of relative vapor pressure differences, in ppm, in figure 4. Moser and Zmaczynski fitted their data to a polynomial equation of the fourth degree in temperature. We, therefore, have included a curve of the differences between the Moser and Zmaczynski equation and eq (17). There is a varying negative difference between the

5 The Moser and Zmaczynski data extend over the temperature range 73 to $130^{\circ} \mathrm{C}$. W have limited our consideration of these data to the temperature span 73 to $103{ }^{\circ} \mathrm{C}$, the upper end corresponding to the highest temperature of the Beattie and Blaisdell data. 
TABLE 4. Ice point and triple point vapor pressures comparisons

\begin{tabular}{|c|c|c|c|c|c|c|c|}
\hline \multirow{2}{*}{ Source } & \multirow{3}{*}{ Reference } & \multicolumn{3}{|c|}{ Ice point, $0^{\circ}$} & \multicolumn{3}{|c|}{ Triple point, $0.01{ }^{\circ} \mathrm{C}$} \\
\hline & & \multirow{2}{*}{\begin{tabular}{|c|}
$\begin{array}{c}\text { Vapor } \\
\text { pressure }^{\text {a }}\end{array}$ \\
pascal \\
610.75
\end{tabular}} & \multicolumn{2}{|c|}{$\begin{array}{c}\text { Difference } \\
\text { Other-Eq (17) }\end{array}$} & \multirow{2}{*}{$\begin{array}{c}\begin{array}{c}\text { Vapor } \\
\text { pressure }\end{array} \\
\text { pascal } \\
611.20\end{array}$} & \multicolumn{2}{|c|}{$\begin{array}{l}\text { Difference } \\
\text { Other-Eq (17) }\end{array}$} \\
\hline $\mathrm{Eq}(17)$ & & & pascal & ppm & & pascal & ppm \\
\hline Experimental Measurements & & & & & & & \\
\hline $\begin{array}{l}\text { Thiesen and Scheel (1900) } \\
\text { Scheel and Heuse (1909) } \\
\text { Scheel and Heuse (1910) } \\
\text { Prytz (1931) } \\
\text { Douslin (1970) }\end{array}$ & $\begin{array}{l}{[41]} \\
{[42]} \\
{[28]} \\
{[43]} \\
{[40]}\end{array}$ & $\begin{array}{l}610.46 \\
610.46 \\
610.11 \\
611.5\end{array}$ & $\begin{array}{l}-0.29 \\
-0.29 \\
-0.64 \\
+0.75\end{array}$ & $\begin{array}{l}-480 \\
-\quad 480 \\
-1050 \\
+1200\end{array}$ & $\begin{array}{l}611.52 \\
612.2\end{array}$ & $\begin{array}{l}+0.32 \\
+1.0\end{array}$ & $\begin{array}{r}+530 \\
+1640\end{array}$ \\
\hline Steam Tables & & & & & & & \\
\hline $\begin{array}{l}\text { IST (1963) } \\
\text { NEL (1964) } \\
\text { IFC (1967) } \\
\text { Keenan, Keyes, et al. (1969) }\end{array}$ & $\begin{array}{l}{[44]} \\
{[44]} \\
{[48]} \\
{[16]}\end{array}$ & $\begin{array}{l}610.8 \\
610.7 \\
610.8 \\
610.8\end{array}$ & $\begin{array}{l}+0.05 \\
-0.05 \\
+0.05 \\
+0.05\end{array}$ & $\begin{array}{l}+\quad 80 \\
-\quad 80 \\
+\quad 80 \\
+\quad 80\end{array}$ & $\begin{array}{l}611.2 \\
611.2 \\
611.2 \\
611.3\end{array}$ & $\begin{array}{r}0.0 \\
0.0 \\
0.0 \\
+0.1\end{array}$ & $\begin{array}{r}0 \\
0 \\
0 \\
+160\end{array}$ \\
\hline
\end{tabular}

a Metastable state; vapor pressure with respect to supercooled water.

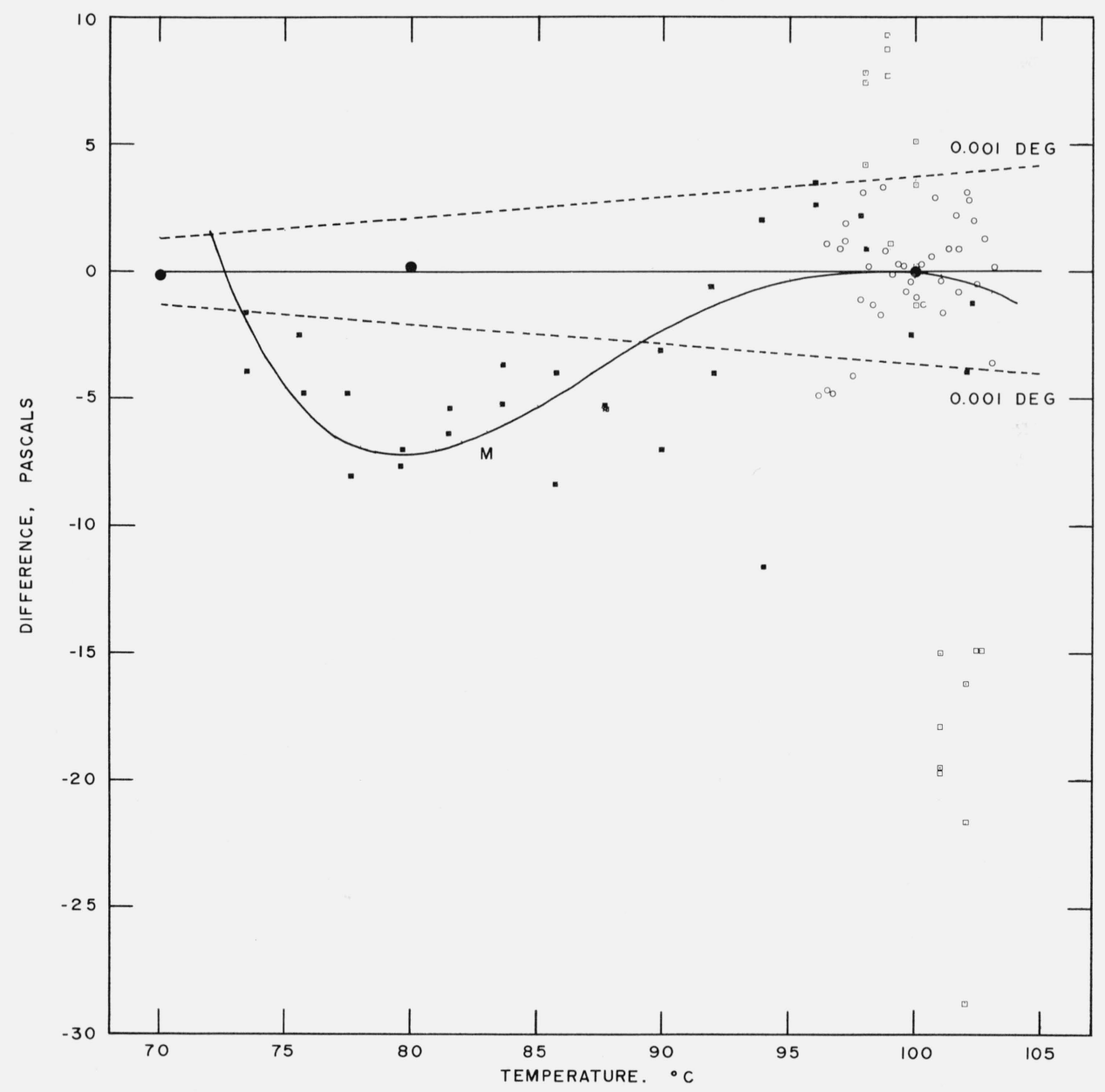

Figure 3. Comparison with experimental data at and below the steam point.

Vapor pressure difference [measurement-eq (17)] in pascals between measurement and eq (17).

- $\begin{aligned} & \text { Stımson } \\ & \text { Moser and Zmaczynski }\end{aligned} \quad$ M Michels, Blaisse, Ten Seldam and Wouters

Beattie and Blaisdell

Dashed lines are difference curves for a temperature deviation of $\pm 0.001 \mathrm{deg}$. 


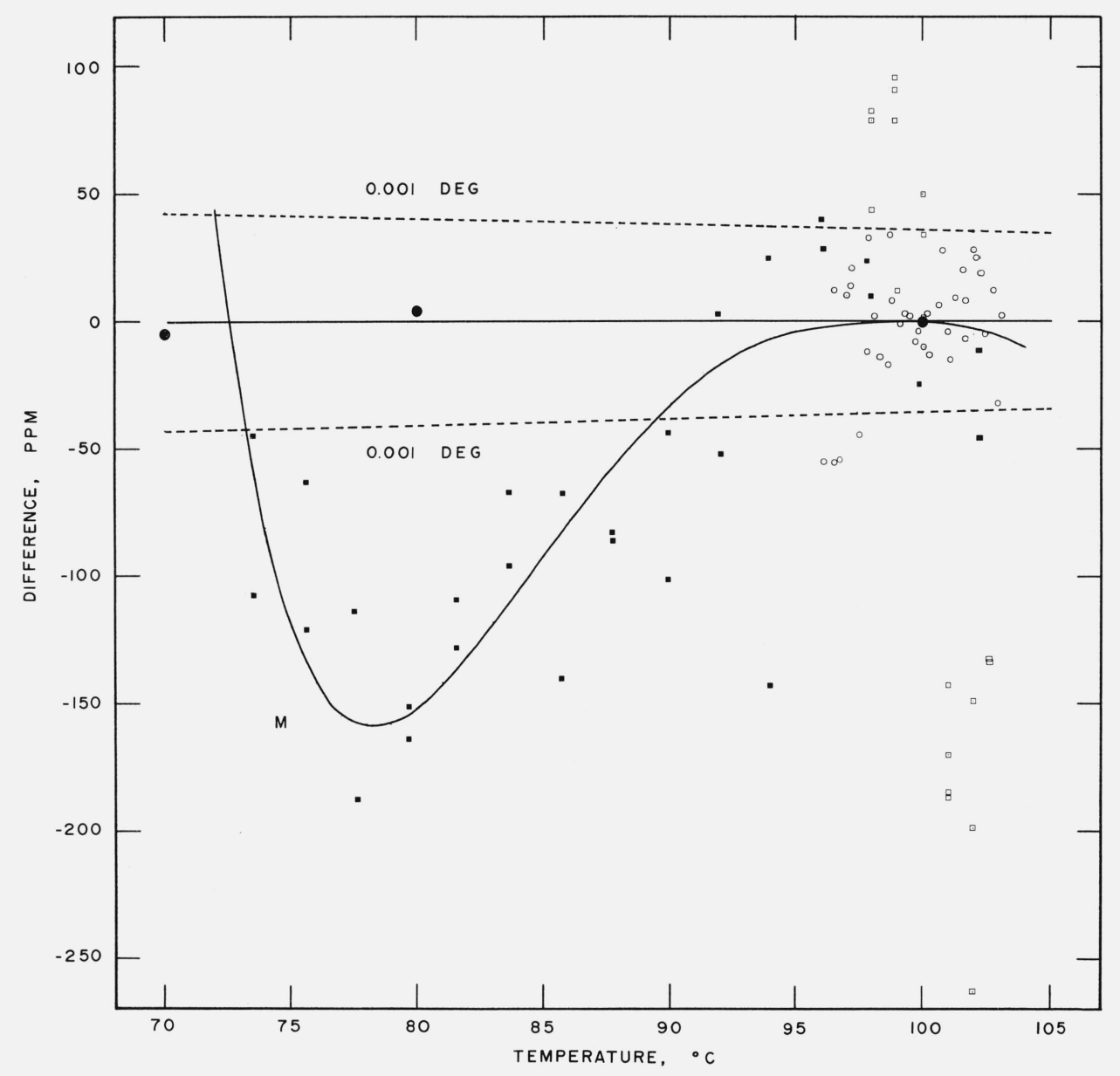

FigURE 4. Comparison with experimental data at and below the steam point.

Relative vapor pressure difference $\left[\frac{\text { measurement }-\mathrm{eq}(17)}{\mathrm{eq}(17)} \times 10^{6}\right]$ in ppm between measurement and eq (17).

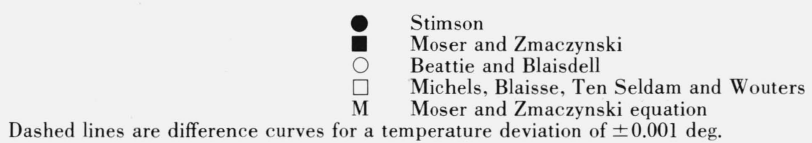

Dashed lines are difference curves for a temperature deviation of $\pm 0.001 \mathrm{deg}$.

Moser and Zmaczynski data and eq (17), reaching a maximum at $78{ }^{\circ} \mathrm{C}$ of -8 pascals $(-190 \mathrm{ppm})$. The Beattie and Blaisdell data cover only the very narrow temperature range of 96 to $103{ }^{\circ} \mathrm{C}$. The differences appear to be random with respect to sign and magnitude. The residual standard deviation (based on $n-7$ degrees of freedom where 7 is the number of coefficients in eq (17)) is 1.55 pascals (17 ppm). The Michels et al. data extend from 98 to $102.6^{\circ} \mathrm{C}$. The differences vary linearly with temperature from about +9.3 pascals $(+100 \mathrm{ppm})$ at $98^{\circ} \mathrm{C}$ to about -27.7 pascals $(-260$ ppm) at $102.6^{\circ} \mathrm{C}$. 


\subsection{Comparisons With Other Formulations}

There have been numerous attempts to formulate vapor pressure equations for the pure water substance. Some of the equations are based on theoretical considerations, others are empirical. Since Sonntag [45] has summarized the extensive literature on the vapor pressure of water, we will not attempt to repeat it here. However, we will compare vapor pressures computed from several formulas which are in current use with vapor pressures computed from eq (17) over the range 0 to $100{ }^{\circ} \mathrm{C}$. We have chosen for this purpose the equations due to Gerry [44, 46], Goff and Gratch [1], American Petroleum Institute [47], 1967 IFC [48], Bridgeman and Aldrich [49], and Gibson and Bruges [50].

The Gerry equation is an empirical formula derived from Physikalisch-Technische Reichsanstalt data. It was adopted by the Third International Conference on Steam Tables in 1934 [51] and has been used in successive formulations of the International Skeleton Tables.

The Goff and Gratch equation is based on integration of the Clausius-Clapeyron equation. It was adopted in 1947 by the Twelfth Conference of Directors of the International Meteorological Organization and by the Joint International Committee on Psychrometric Data in 1949 [19]. Tables of vapor pressure were published in the ASHRAE Guide [52] and in the Smithsonian Meteorological Tables [53]. Goff transposed this equation from the absolute ITS-48 to the absolute IPTS-48 [1] in which form it was adopted by the World Meteorological Organization [54]. It is this latter version which we use for our comparison.

The American Petroleum Institute Research Project 44 values of vapor pressure were calculated from the Antoine equation using several sets of constants, each set valid for a narrow range of temperatures. These constants were derived by fitting the Antoine equation to vapor pressures obtained from the Osborne and Meyers formulation [31] to which were added the "Cragoe-Stimson" corrections. The latter were obtained from vapor pressure measurements made at NBS in 1942 which had received limited private circulation but had remained unpublished until 1967 [50]. These 1942 measurements, augmented by a more extensive series of measurements made during the years 1947 through 1949, were finally published in 1969 by Stimson [2].

At the Sixth International Conference on the Properties of Steam an International Formulation Committee (IFC) was established to develop for use with computers a thermodynamically consistent formulation of the properties of steam within the tolerances of the International Skeleton Table of 1963. The result was the 1967 IFC Formulation for Industrial Use. Values of vapor pressure calculated using the vapor pressure equation given by the 1967 IFC are tabulated in the 1967 ASME Steam Tables [48].

In 1964, Bridgeman and Aldrich published an equation and tables of vapor pressure for water. Keenan et al. [16] used the Bridgeman and Aldrich values to derive an alternate equation. We used the logarithm of the vapor pressure given by Bridgeman and Aldrich in their tables [49] and converted this to vapor pressure.

Gibson and Bruges [50] derived two empirical vapor pressure equations using Chebyshev polynomials. One equation, which we will designate by $a$, reproduces the entries in table 1 of the 1964. NEL Steam Tables [44] which, in turn, are derived from the Gerry equation $[44,46]$, for 0 to $100{ }^{\circ} \mathrm{C}$ and from the Osborne and Meyers formulation [31] above $100{ }^{\circ} \mathrm{C}$. The second equation, which we will designate by $b$, is based on APIRP-44 values [47] between 0 and $100{ }^{\circ} \mathrm{C}$, and on the Osborne and Meyers formulation above $100{ }^{\circ} \mathrm{C}$.

We have calculated the differences in vapor pressure between each of the above formulations and eq (17) and plotted these in pascals on figure 5 and in ppm on figure 6. Also included on these figures are the differences between Stimson's measurements and eq (17). These figures show the degree of accord of the several formulations with eq (17) as well as with the Stimson vapor pressures.

\subsection{Conversion to IPTS-68}

In 1968 the International Practical Temperature Scale of 1968 (IPTS-68) was adopted [32] as a replacement for IPTS-48. We have converted eq (17) from IPTS-48 to IPTS-68. Let the difference between these two scales be designated by $\mu$, that is,

$$
T_{68}-T_{48}=\mu .
$$

Douglas [55] has shown that from 0 to $630.74{ }^{\circ} \mathrm{C}, \mu$ is given by

$$
\mu=\frac{4.904\left(10^{-7}\right) t_{68}\left(t_{68}-100\right)}{1-2.939\left(10^{-4}\right) t_{68}}+\varphi\left(t_{68}\right)
$$

where

$$
\begin{array}{r}
\varphi\left(t_{68}\right)=0.045\left(\frac{t_{68}}{100}\right)\left(\frac{t_{68}}{100}-1\right)\left(\frac{t_{68}}{419.58}-1\right) \\
\left(\frac{t_{68}}{630.74}-1\right)
\end{array}
$$

and $t_{68}$ is in Celsius degrees on IPTS-68. Expanding eq (20) into a polynomial, converting $t_{68}$ to $T_{68}$, where $T_{68}=t_{68}+273.15$, and neglecting higher order terms in $T_{68}$, we obtain

$$
\mu=\sum_{i=0}^{4} \alpha_{i} T_{68}^{i}
$$

where $\quad \alpha_{0}=1.1314914, \quad \alpha_{1}=-9.9294171 \times 10^{-3}$, $\alpha_{2}=3.0306087 \times 10^{-5}, \quad \alpha_{3}=-3.9043313 \times 10^{-8}$ and $\alpha_{4}=1.7044968 \times 10^{-11}$. Equations (19) and (22) are now substituted into eq (17) which, after appropriate algebraic manipulations, and neglecting higher order terms in $\mu$, becomes

$$
\ln p=\sum_{i=0}^{9} F_{i} T_{68}^{i-2}+B \ln T_{68}
$$




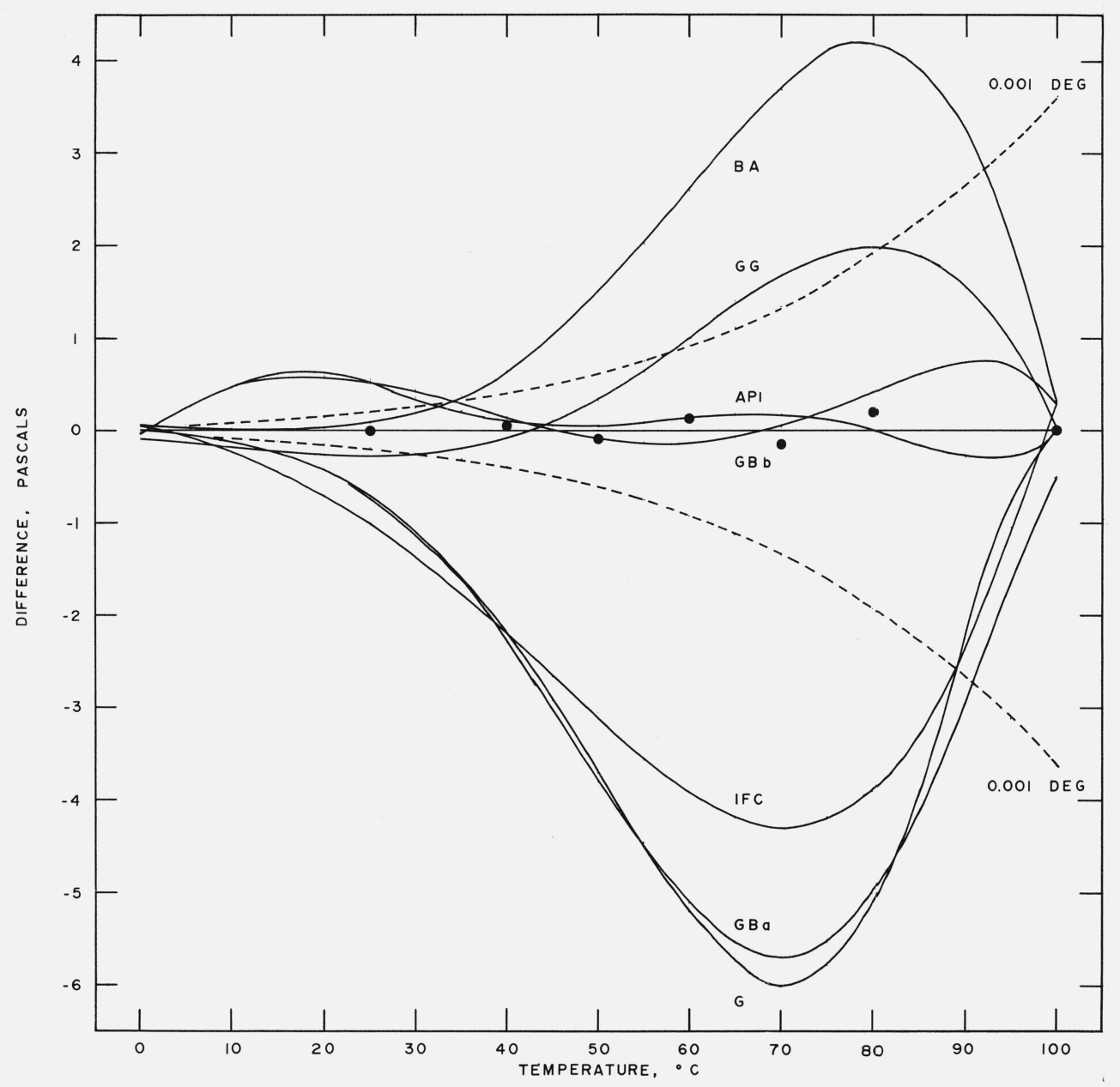

Figure 5. Comparison with other formulations.

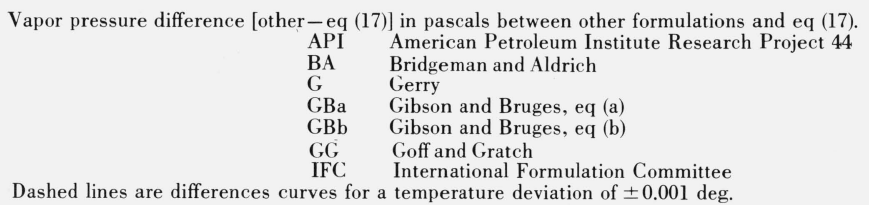

where $F_{0}=-8.49922 \times 10^{3}, F_{1}=-7.4231865 \times 10^{3}$, $F_{2}=96.1635147, \quad F_{3}=2.4917646 \times 10^{-2}$, $F_{4}=-1.3160119 \times 10^{-5}, \quad F_{5}=-1.1460454 \times 10^{-8}$, $F_{6}=2.1701289 \times 10^{-11}, \quad F_{7}=-3.610258 \times 10^{-15}$, $F_{8}=3.8504519 \times 10^{-18}, \quad F_{9}=-1.4317 \times 10^{-21}$, and $B=-12.150799$.

In the range 0 to $100{ }^{\circ} \mathrm{C}$, the numerical values of temperature on IPTS-48 are larger than on IPTS- 68. Therefore, at the same numerical values of temperature, the corresponding vapor pressures are smaller on IPTS-48. For example, at $t_{48}=50^{\circ} \mathrm{C}$, the vapor pressure, calculated by eq (17), is 12338.39 pascals whereas at $t_{68}=50{ }^{\circ} \mathrm{C}$, the vapor pressure, calculated by eq (23), is 12344.73 pascals.

We investigated the feasibility of simplifying eq (23). We fitted the equation

$$
\ln p=\sum_{i=0}^{n} G_{i} T_{6 \overline{8}}^{1}+H \ln T_{68}
$$

for $2 \equiv n ₹ 4$ by the method of least squares to values of vapor pressure generated at $1-\mathrm{deg}$ intervals from 0 to $100{ }^{\circ} \mathrm{C}$ by eq (23). The results are comparable to that obtained for $T_{48}$. We, therefore, only present the results for $n=3$ where the largest difference between eqs (23) and (24) is $8 \mathrm{ppm}$. The coefficients are as follows: $G_{0}=-6898.2434, \quad G_{1}=59.38385, \quad G_{2}=-$ $5.797662 \times 10^{-3}, G_{3}=6.2223854 \times 10^{-6}$, and $H=-$ 4.7406885. Equation (24) on IPTS-68 with these coefficients is equivalent to eq (18b) on IPTS-48.

\subsection{Tabulations}

Using eqs (17) and (23) we computed vapor pressure in pascals, as a function of temperature, in degrees Celsuis, at 0.1-deg intervals, from 0 to $100{ }^{\circ} \mathrm{C}$, These computed values are given in table 5 on IPTS-48 and in table 6 on IPTS-68. 


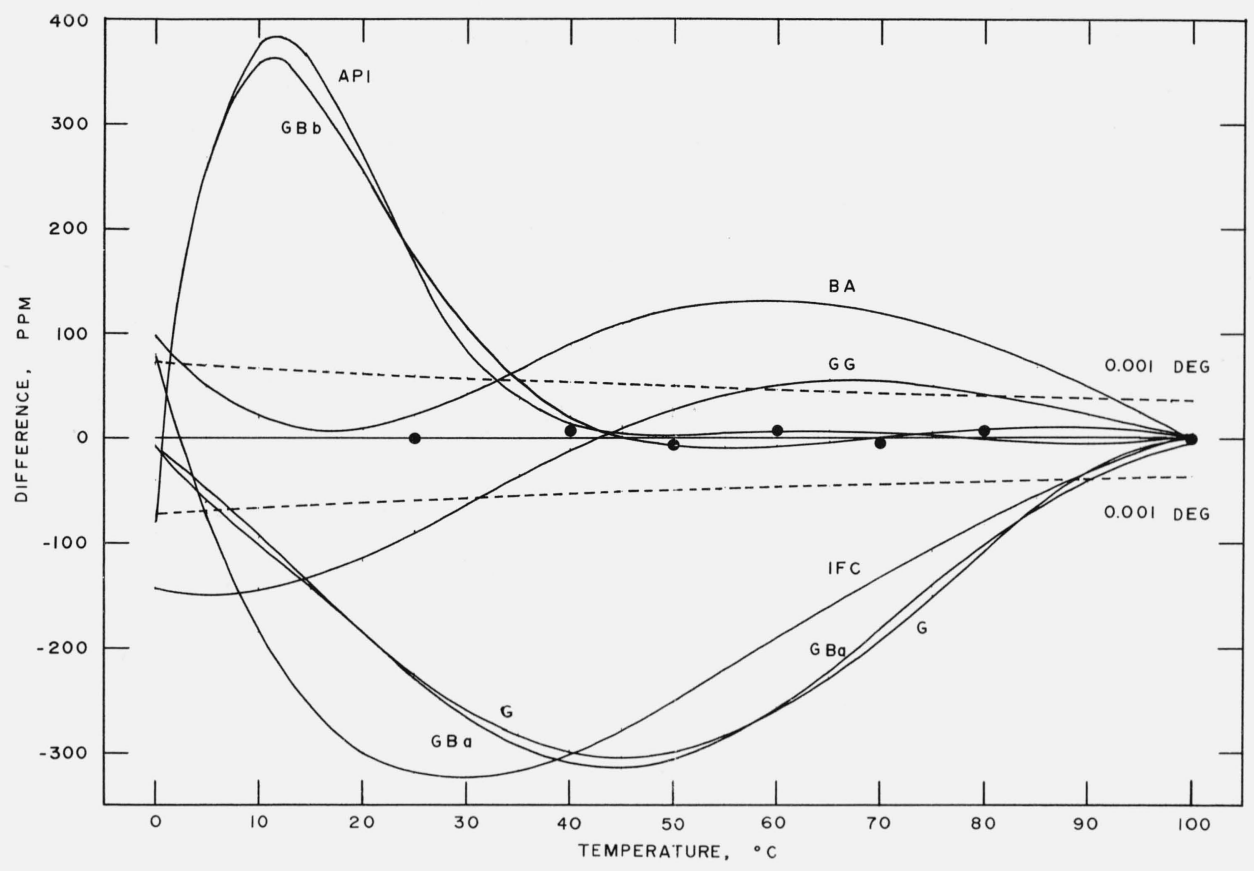

Figure 6. Comparison with other formulations.

Relative vapor pressure difference $\left[\frac{\text { other }-\mathrm{eq}(17)}{\mathrm{eq}(17)} \times 10^{6}\right]$ in ppm between other formulations and eq (17).

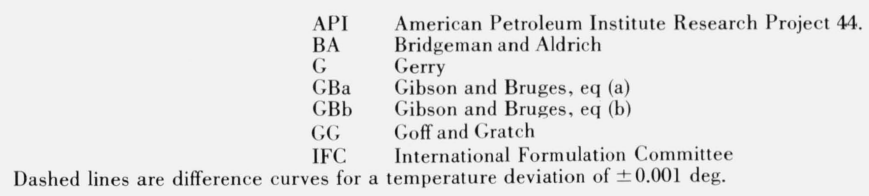


TABLE 5. Saturation vapor pressure over water (IPTS-48)

\begin{tabular}{|c|c|c|c|c|c|c|c|c|c|c|}
\hline $\begin{array}{c}\text { Temperature } \\
\text { deg C }\end{array}$ & 0.0 & 0.1 & 0.2 & 0.3 & 0.4 & 0.5 & 0.6 & 0.7 & 0.8 & 0.9 \\
\hline & pascals & pascals & pascals & pascals & pascals & pascals & pascals & pascals & pascals & pascals \\
\hline 0 & a 610.752 & 615.205 & 619.686 & 624.196 & 628.736 & 633.304 & 637.902 & 642.529 & 647.187 & 651.874 \\
\hline 1 & 656.591 & 661.338 & 666.116 & 670.924 & 675.763 & 680.633 & 685.534 & 690.466 & 695.429 & 700.425 \\
\hline 2 & 705.451 & 710.510 & 715.601 & 720.724 & 725.879 & 731.068 & 736.289 & 741.542 & 746.829 & 752.150 \\
\hline 3 & 757.504 & 762.891 & 768.313 & 773.768 & 779.258 & 784.782 & 790.341 & 795.934 & 801.563 & 807.227 \\
\hline 4 & 812.926 & 818.660 & 824.431 & 830.237 & 836.079 & 841.958 & 847.873 & 853.825 & 859.814 & 865.840 \\
\hline 5 & 871.903 & 878.004 & 884.142 & 890.318 & 896.533 & 902.785 & 909.076 & 915.406 & 921.775 & 928.183 \\
\hline 6 & 934.630 & 941.117 & 947.643 & 954.209 & 960.816 & 967.462 & 974.150 & 980.878 & 987.647 & 994.457 \\
\hline 7 & 1001.31 & 1008.20 & 1015.14 & 1022.11 & 1029.13 & 1036.20 & 1043.30 & 1050.45 & 1057.64 & 1064.87 \\
\hline 8 & 1072.15 & 1079.47 & 1086.84 & 1094.25 & 1101.70 & 1109.20 & 1116.75 & 1124.34 & 1131.97 & 1139.65 \\
\hline 9 & 1147.38 & 1155.15 & 1162.97 & 1170.83 & 1178.75 & 1186.71 & 1194.71 & 1202.77 & 1210.87 & 1219.02 \\
\hline 10 & 1227.22 & 1235.46 & 1243.76 & 1252.10 & 1260.50 & 1268.94 & 1277.43 & 1285.98 & 1294.57 & 1303.22 \\
\hline 11 & 1311.91 & 1320.66 & 1329.45 & 1338.30 & 1347.20 & 1356.15 & 1365.16 & 1374.22 & 1383.33 & 1392.49 \\
\hline 12 & 1401.71 & 1410.98 & 1420.30 & 1429.68 & 1439.11 & 1448.60 & 1458.14 & 1467.74 & 1477.39 & 1487.10 \\
\hline 13 & 1496.87 & 1506.69 & 1516.57 & 1526.50 & 1536.49 & 1546.54 & 1556.65 & 1566.81 & 1577.04 & 1587.32 \\
\hline 14 & 1597.66 & 1608.06 & 1618.52 & 1629.04 & 1639.62 & 1650.25 & 1660.95 & 1671.72 & 1682.54 & 1693.42 \\
\hline 15 & 1704.37 & 1715.37 & 1726.44 & 1737.57 & 1748.77 & 1760.03 & 1771.35 & 1782.74 & 1794.19 & 1805.70 \\
\hline 16 & 1817.28 & 1828.92 & 1840.63 & 1852.41 & 1864.25 & 1876.16 & 1888.13 & 1900.18 & 1912.28 & 1924.46 \\
\hline 17 & 1936.70 & 1949-02 & 1961.40 & 1973.85 & 1986.37 & 1998.96 & 2011.62 & 2024.34 & 2037.14 & 2050.01 \\
\hline 18 & 2062.96 & 2075.97 & 2089.05 & 2102.21 & 2115.44 & 2128.74 & 2142.12 & 2155.57 & 2169.09 & 2182.69 \\
\hline 19 & 2196.36 & 2210.11 & 2223.93 & 2237.83 & 2251.80 & 2265.85 & 2279.98 & 2294.18 & 2308.47 & 2322.83 \\
\hline 20 & 2337.26 & 2351.78 & 2366.37 & 2381.05 & 2395.80 & 2410.63 & 2425.55 & 2440.54 & 2455.62 & 2470.77 \\
\hline 21 & 2486.01 & 2501.33 & 2516.73 & 2532.22 & 2547.79 & 2563.44 & 2579.18 & 2595.00 & 2610.90 & 2626.90 \\
\hline 22 & 2642.97 & 2659.13 & 2675.38 & 2691.72 & 2708.14 & 2724.65 & 2741.25 & 2757.94 & 2774.71 & 2791.57 \\
\hline 23 & 2808.53 & 2825.57 & 2842.70 & 2859.93 & 2877.24 & 2894.65 & 2912.15 & 2929.74 & 2947.42 & 2965.20 \\
\hline 24 & 2983.07 & 3001.03 & 3019.09 & 3037.24 & 3055.49 & 3073.84 & 3092.28 & 3110.81 & 3129.44 & 3148.17 \\
\hline 25 & 3167.00 & 3185.93 & 3204.95 & 3224.08 & 3243.30 & 3262.62 & 3282.05 & 3301.57 & 3321.20 & 3340.92 \\
\hline 26 & 3360.75 & 3380.68 & 3400.72 & 3420.86 & 3441.10 & 3461.44 & 3481.90 & 3502.45 & 3523.11 & 3543.88 \\
\hline 27 & 3564.75 & 3585.74 & 3606.82 & 3628.02 & 3649.33 & 3670.74 & 3692.26 & 3713.90 & 3735.64 & 3757.49 \\
\hline 28 & 3779.46 & 3801.54 & 3823.73 & 3846.03 & 3868.44 & 3890.97 & 3913.61 & 3936.37 & 3959.24 & 3982.23 \\
\hline 29 & 4005.33 & 4028.56 & 4051.89 & 4075.35 & 4098.92 & 4122.61 & 4146.42 & 4170.35 & 4194.40 & 4218.57 \\
\hline 30 & 4242.86 & 4267.28 & 4291.81 & 4316.47 & 4341.25 & 4366.15 & 4391.18 & 4416.33 & 4441.61 & 4467.01 \\
\hline 31 & 4492.54 & 4518.20 & 4543.98 & 4569.89 & 4595.93 & 4622.10 & 4648.40 & 4674.82 & 4701.38 & 4728.07 \\
\hline 32 & 4754.89 & 4781.84 & 4808.93 & 4836.14 & 4863.49 & 4890.98 & 4918.60 & 4946.35 & 4974.24 & 5002.27 \\
\hline 33 & 5030.44 & 5058.74 & 5087.18 & 5115.76 & 5144.47 & 5173.33 & 5202.33 & 5231.47 & 5260.74 & 5290.17 \\
\hline 34 & 5319.73 & 5349.44 & 5379.29 & 5409.28 & 5439.42 & 5469.71 & 5500.14 & 5530.72 & 5561.45 & 5592.32 \\
\hline 35 & 5623.34 & 5654.51 & 5685.83 & 5717.30 & 5748.92 & 5780.69 & 5812.62 & 5844.70 & 5876.93 & 5909.31 \\
\hline 36 & 5941.85 & 5974.54 & 6007.39 & 6040.40 & 6073.56 & 6106.88 & 6140.36 & 6173.99 & 6207.79 & 6241.75 \\
\hline 37 & 6275.86 & 6310.14 & 6344.58 & 6379.18 & 6413.95 & 6448.87 & 6483.97 & 6519.23 & 6554.65 & 6590.24 \\
\hline 38 & 6626.00 & 6661.92 & 6698.02 & 6734.28 & 6770.71 & 6807.31 & 6844.08 & 6881.03 & 6918.15 & 6955.44 \\
\hline 39 & 6992.90 & 7030.54 & 7068.35 & 7106.34 & 7144.50 & 7182.84 & 7221.36 & 7260.06 & 7298.93 & 7337.99 \\
\hline 40 & 7377.22 & 7416.64 & 7456.24 & 7496.02 & 7535.98 & 7576.13 & 7616.46 & 7656.98 & 7697.68 & 7738.57 \\
\hline 41 & 7779.65 & 7820.92 & 7862.37 & 7904.01 & 7945.85 & 7987.87 & 8030.09 & 8072.50 & 8115.10 & 8157.89 \\
\hline 42 & 8200.88 & 8244.07 & 8287.45 & 8331.02 & 8374.80 & 8418.77 & 8462.94 & 8507.31 & 8551.89 & 8596.66 \\
\hline 43 & 8641.63 & 8686.81 & 8732.19 & 8777.78 & 8823.57 & 8869.56 & 8915.76 & 8962.17 & 9008.79 & 9055.61 \\
\hline 44 & 9102.65 & 9149.89 & 9197.35 & 9245.02 & 9292.90 & 9340.99 & 9389.30 & 9437.82 & 9486.56 & 9535.51 \\
\hline 45 & 9584.69 & 9634.08 & 9683.68 & 9733.51 & 9783.56 & 9833.83 & 9884.32 & 9935.04 & 9985.98 & 10037.14 \\
\hline 46 & 10088.53 & 10140.14 & 10191.99 & 10244.06 & 10296.35 & 10348.88 & 10401.64 & 10454.63 & 10507.85 & 10561.30 \\
\hline 47 & 10614.98 & 10668.91 & 10723.06 & 10777.45 & 10832.08 & 10886.95 & 10942.05 & 10997.40 & 11052.98 & 11108.81 \\
\hline 48 & 11164.88 & 11221.19 & 11277.74 & 11334.54 & 11391.58 & 11448.88 & 11506.41 & 11564.20 & 11622.23 & 11680.52 \\
\hline 49 & 11739.05 & 11797.84 & 11856.88 & 11916.17 & 11975.72 & 12035.52 & 12095.58 & 12155.89 & 12216.47 & 12277.30 \\
\hline 50 & 12338.39 & 12399.74 & 12461.35 & 12523.23 & 12585.37 & 12647.77 & 12710.44 & 12773.37 & 12836.57 & 12900.04 \\
\hline 51 & 12963.77 & 13027.78 & 13092.06 & 13156.61 & 13221.43 & 13286.52 & 13351.89 & 13417.54 & 13483.46 & 13549.66 \\
\hline 52 & 13616.13 & 13682.89 & 13749.92 & 13817.24 & 13884.84 & 13952.72 & 14020.88 & 14089.33 & 14158.07 & 14227.09 \\
\hline 53 & 14296.40 & 14366.00 & 14435.89 & 14506.07 & 14576.54 & 14647.30 & 14718.36 & 14789.71 & 14861.36 & 14933.31 \\
\hline 54 & 15005.55 & 15078.09 & 15150.93 & 15224.07 & 15297.51 & 15371.26 & 15445.31 & 15519.66 & 15594.32 & 15669.29 \\
\hline 55 & 15744.56 & 15820.15 & 15896.04 & 15972.25 & 16048.76 & 16125.59 & 16202.73 & 16280.19 & 16357.97 & 16436.06 \\
\hline 56 & 16514.47 & 16593.19 & 16672.24 & 16751.61 & 16831.31 & 16911.32 & 16991.66 & 17072.33 & 17153.32 & 17234.64 \\
\hline 57 & 17316.29 & 17398.27 & 17480.58 & 17563.22 & 17646.20 & 17729.51 & 17813.15 & 17897.13 & 17981.45 & 18066.11 \\
\hline 58 & 18151.11 & 18236.45 & 18322.12 & 18408.15 & 18494.51 & 18581.23 & 18668.29 & 18755.69 & 18843.45 & 18931.55 \\
\hline 59 & 19020.01 & 19108.81 & 19197.97 & 19287.49 & 19377.36 & 19467.58 & 19558.17 & 19649.11 & 19740.41 & 19832.08 \\
\hline
\end{tabular}


TABLE 5. Saturation vapor pressure over water (IPTS-48)-Continued

\begin{tabular}{|c|c|c|c|c|c|c|c|c|c|c|}
\hline $\begin{array}{c}\text { Temperature } \\
\operatorname{deg} C\end{array}$ & 0.0 & 0.1 & 0.2 & 0.3 & 0.4 & 0.5 & 0.6 & 0.7 & 0.8 & 0.9 \\
\hline & pascals & pascals & pascals & pascals & pascals & pascals & pascals & pascals & pascals & pascals \\
\hline 60 & 19924.10 & 20016.49 & 20109.25 & 20202.37 & 20295.86 & 20389.71 & 20483.93 & 20578.53 & 20673.50 & 20768.83 \\
\hline 61 & 20864.55 & 20960.64 & 21057.10 & 21153.94 & 21251.16 & 21348.76 & 21446.74 & 21545.11 & 21643.86 & 21742.99 \\
\hline 62 & 21842.51 & 21942.41 & 22042.70 & 22143.39 & 22244.46 & 22345.93 & 22447.79 & 22550.04 & 22652.69 & 22755.73 \\
\hline 63 & 22859.18 & 22963.02 & 23067.27 & 23171.91 & 23276.96 & 23382.41 & 23488.27 & 23594.54 & 23701.21 & 23808.29 \\
\hline 64 & 23915.79 & 24023.69 & 24132.01 & 24240.75 & 24349.90 & 24459.46 & 24569.45 & 24679.85 & 24790.67 & 24901.92 \\
\hline 65 & 25013.59 & 25125.69 & 25238.21 & 25351.15 & 25464.53 & 25578.34 & 25692.58 & 25807.25 & 25922.35 & 26037.89 \\
\hline 66 & 26153.87 & 26270.28 & 26387.13 & 26504.43 & 26622.16 & 26740.34 & 26858.96 & 26978.03 & 27097.54 & 27217.51 \\
\hline 67 & 27337.92 & 27458.79 & 27580.10 & 27701.88 & 27824.10 & 27946.79 & 28069.93 & 28193.53 & 28317.59 & 28442.11 \\
\hline 68 & 28567.10 & 28692.55 & 28818.47 & 28944.85 & 29071.71 & 29199.03 & 29326.83 & 29455.10 & 29583.84 & 29713.06 \\
\hline 69 & 29842.76 & 29972.94 & 30103.59 & 30234.73 & 30366.35 & 30498.46 & 30631.05 & 30764.13 & 30897.70 & 31031.76 \\
\hline 70 & 31166.31 & 31301.35 & 31436.89 & 31572.92 & 31709.45 & 31846.48 & 31984.01 & 32122.04 & 32260.57 & 32399.61 \\
\hline 71 & 32539.16 & 32679.21 & 32819.77 & 32960.84 & 33102.43 & 33244.53 & 33387.14 & 33530.27 & 33673.92 & 33818.08 \\
\hline 72 & 33962.77 & 34107.98 & 34253.72 & 34399.98 & 34546.76 & 34694.08 & 34841.92 & 34990.30 & 35139.21 & 35288.65 \\
\hline 73 & 35438.63 & 35589.15 & 35740.21 & 35891.80 & 36043.94 & 36196.63 & 36349.86 & 36503.63 & 36657.95 & 36812.83 \\
\hline 74 & 36968.25 & 37124.23 & 37280.77 & 37437.85 & 37595.50 & 37753.71 & 37912.47 & 38071.80 & 38231.70 & 38392.16 \\
\hline 75 & 38553.18 & 38714.78 & 38876.94 & 39039.68 & 39202.99 & 39366.88 & 39531.34 & 39696.38 & 39862.01 & 40028.21 \\
\hline 76 & 40195.00 & 40362.37 & 40530.33 & 40698.87 & 40868.01 & 41037.73 & 41208.06 & 41378.97 & 41550.48 & 41722.59 \\
\hline 77 & 41895.30 & 42068.61 & 42242.52 & 42417.04 & 42592.16 & 42767.90 & 42944.24 & 43121.19 & 43298.76 & 43476.94 \\
\hline 78 & 43655.73 & 43835.15 & 44015.18 & 44195.84 & 44377.11 & 44559.02 & 44741.55 & 44924.71 & 45108.49 & 45292.91 \\
\hline 79 & 45477.96 & 45663.65 & 45849.98 & 46036.94 & 46224.54 & 46412.79 & 46601.67 & 46791.21 & 46981.39 & 47172.22 \\
\hline 80 & 47363.70 & 47555.83 & 47748.62 & 47942.06 & 48136.16 & 48330.92 & 48526.34 & 48722.42 & 48919.17 & 49116.58 \\
\hline 81 & 49314.67 & 49513.42 & 49712.84 & 49912.94 & 50113.71 & 50315.16 & 50517.29 & 50720.10 & 50923.59 & 51127.77 \\
\hline 82 & 51332.63 & 51538.18 & 51744.43 & 51951.36 & 52158.98 & 52367.30 & 52576.32 & 52786.04 & 52996.46 & 53207.58 \\
\hline 83 & 53419.40 & 53631.93 & 53845.17 & 54059.12 & 54273.78 & 54489.16 & 54705.25 & 54922.05 & 55139.58 & 55357.83 \\
\hline 84 & 55576.80 & 55796.50 & 56016.92 & 56238.07 & 56459.95 & 56682.57 & 56905.92 & 57130.00 & 57354.82 & 57580.39 \\
\hline 85 & 57806.69 & 58033.74 & 58261,54 & 58490.08 & 58719.37 & 58949.42 & 59180.21 & 59411.77 & 59644.08 & 59877.15 \\
\hline 86 & 60110.98 & 60345.57 & 60580.93 & 60817.06 & 61053.95 & 61291.62 & 61530.06 & 61769.27 & 62009.26 & 62250.03 \\
\hline 87 & 62491.58 & 62733.92 & 62977.04 & 63220.94 & 63465.64 & 63711.12 & 63957.40 & 64204.47 & 64452.34 & 64701.01 \\
\hline 88 & 64950.48 & 65200.75 & 65451.82 & 65703.71 & 65956.40 & 66209.90 & 66464.22 & 66719.35 & 66975.30 & 67232.06 \\
\hline 89 & 67489.65 & 67748.06 & 68007.30 & 68267.36 & 68528.26 & 68789.98 & 69052.54 & 69315.93 & 69580.16 & 69845.23 \\
\hline 90 & 70111.14 & 70377.90 & 70645.50 & 70913.95 & 71183.25 & 71453.40 & 71724.41 & 71996.27 & 72268.99 & 72542.57 \\
\hline 91 & 72817.02 & 73092.33 & 73368.50 & 73645.55 & 73923.46 & 74202.25 & 74481.92 & 74762.46 & 75043.88 & 75326.18 \\
\hline 92 & 75609.37 & 75893.44 & 76178.41 & 76464.26 & 76751.00 & 77038.64 & 77327.18 & 77616.62 & 77906.95 & 78198.19 \\
\hline 93 & 78490.34 & 78783.39 & 79077.36 & 79372.24 & 79668.03 & 79964.73 & 80262.36 & 80560.91 & 80860.38 & 81160.77 \\
\hline 94 & 81462.10 & 81764.35 & 82067.53 & 82371.65 & 82676.71 & 82982.71 & 83289.64 & 83597.52 & 83906.35 & 84216.12 \\
\hline 95 & 84526.84 & 84838.51 & 85151.14 & 85464.73 & 85779.28 & 86094.78 & 86411.25 & 86728.69 & 87047.09 & 87366.47 \\
\hline 96 & 87686.81 & 88008.13 & 88330.43 & 88653.71 & 88977.97 & 89303.22 & 89629.45 & 89956.67 & 90284.88 & 90614.09 \\
\hline 97 & 90944.29 & 91275.49 & 91607.69 & 91940.89 & 92275.10 & 92610.31 & 92946.53 & 93283.77 & 93622.02 & 93961.29 \\
\hline 98 & 94301.58 & 94642.88 & 94985.22 & 95328.58 & 95672.96 & 96018.38 & 96364.83 & 96712.32 & 97060.85 & 97410.41 \\
\hline 99 & 97761.02 & 98112.68 & 98465.38 & 98819.13 & 99173.94 & 99529.80 & 99886.71 & 100244.69 & 100603.73 & 100963.83 \\
\hline 100 & 101325.01 & & & & & & & & & \\
\hline
\end{tabular}


TABLE 6. Saturation vapor pressure over water (IPTS-68)

\begin{tabular}{|c|c|c|c|c|c|c|c|c|c|c|}
\hline $\begin{array}{c}\text { Temperature } \\
\operatorname{deg} C\end{array}$ & 0.0 & 0.1 & 0.2 & 0.3 & 0.4 & 0.5 & 0.6 & 0.7 & 0.8 & 0.9 \\
\hline & pascals & pascals & pascals & pascals & pascals & pascals & pascals & pascals & pascals & pascals \\
\hline 0 & a 610.752 & 615.207 & 619.690 & 624.203 & 628.744 & 633.315 & 637.916 & 642.545 & 647.205 & 651.894 \\
\hline 1 & 656.614 & 661.364 & 666.144 & 670.955 & 675.796 & 680.669 & 685.572 & 690.507 & 695.473 & 700.471 \\
\hline 2 & 705.500 & 710.562 & 715.655 & 720.781 & 725.939 & 731.130 & 736.354 & 741.611 & 746.901 & 752.224 \\
\hline 3 & 757.581 & 762.971 & 768.396 & 773.854 & 779.347 & 784.874 & 790.436 & 796.033 & 801.664 & 807.331 \\
\hline 4 & 813.034 & 818.771 & 824.545 & 830.355 & 836.200 & 842.082 & 848.001 & 853.956 & 859.948 & 865.978 \\
\hline 5 & 872.045 & 878.149 & 884.291 & 890.470 & 896.688 & 902.945 & 909.239 & 915.573 & 921.945 & 928.357 \\
\hline 6 & 934.808 & 941.298 & 947.828 & 954.399 & 961.009 & 967.660 & 974.351 & 981.083 & 987.856 & 994.670 \\
\hline 7 & 1001.53 & 1008.42 & 1015.36 & 1022.34 & 1029.37 & 1036.43 & 1043.54 & 1050.70 & 1057.89 & 1065.13 \\
\hline 8 & 1072.41 & 1079.74 & 1087.11 & 1094.52 & 1101.98 & 1109.48 & 1117.03 & 1124.63 & 1132.27 & 1139.95 \\
\hline 9 & 1147.68 & 1155.46 & 1163.28 & 1171.15 & 1179.07 & 1187.04 & 1195.05 & 1203.11 & 1211.21 & 1219.37 \\
\hline 10 & 1227.57 & 1235.83 & 1244.13 & 1252.48 & 1260.88 & 1269.32 & 1277.82 & 1286.37 & 1294.97 & 1303.62 \\
\hline 11 & 1312.32 & 1321.07 & 1329.87 & 1338.73 & 1347.63 & 1356.59 & 1365.60 & 1374.67 & 1383.78 & 1392.95 \\
\hline 12 & 1402.17 & 1411.45 & 1420.78 & 1430.16 & 1439.60 & 1449.10 & 1458.64 & 1468.25 & 1477.91 & 1487.62 \\
\hline 13 & 1497.39 & 1507.22 & 1517.11 & 1527.05 & 1537.04 & 1547.10 & 1557.21 & 1567.39 & 1577.62 & 1587.90 \\
\hline 14 & 1598.25 & 1608.66 & 1619.12 & 1629.65 & 1640.24 & 1650.88 & 1661.59 & 1672.36 & 1683.18 & 1694.08 \\
\hline 15 & 1705.03 & 1716.04 & 1727.12 & 1738.26 & 1749.46 & 1760.73 & 1772.06 & 1783.45 & 1794.91 & 1806.43 \\
\hline 16 & 1818.01 & 1829.67 & 1841.38 & 1853.17 & 1865.02 & 1876.93 & 1888.91 & 1900.96 & 1913.08 & 1925.27 \\
\hline 17 & 1937.52 & 1949.84 & 1962.23 & 1974.69 & 1987.21 & 1999.81 & 2012.48 & 2025.21 & 2038.02 & 2050.90 \\
\hline 18 & 2063.85 & 2076.87 & 2089.97 & 2103.13 & 2116.37 & 2129.68 & 2143.07 & 2156.53 & 2170.06 & 2183.66 \\
\hline 19 & 2197.34 & 2211.10 & 2224.93 & 2238.84 & 2252.82 & 2266.88 & 2281.02 & 2295.23 & 2309.52 & 2323.89 \\
\hline 20 & 2338.34 & 2352.86 & 2367.47 & 2382.15 & 2396.91 & 2411.76 & 2426.68 & 2441.68 & 2456.77 & 2471.93 \\
\hline 21 & 2487.18 & 2502.51 & 2517.93 & 2533.42 & 2549.00 & 2564.66 & 2580.41 & 2596.24 & 2612.16 & 2628.16 \\
\hline 22 & 2644.25 & 2660.42 & 2676.68 & 2693.02 & 2709.46 & 2725.98 & 2742.59 & 2759.28 & 2776.07 & 2792.94 \\
\hline 23 & 2809.91 & 2826.96 & 2844.11 & 2861.34 & 2878.67 & 2896.09 & 2913.60 & 2931.20 & 2948.89 & 2966.68 \\
\hline 24 & 2984.56 & 3002.54 & 3020.61 & 3038.77 & 3057.03 & 3075.39 & 3093.84 & 3112.39 & 3131.03 & 3149.78 \\
\hline 25 & 3168.62 & 3187.55 & 3206.59 & 3225.73 & 3244.96 & 3264.30 & 3283.73 & 3303.27 & 3322.91 & 3342.65 \\
\hline 26 & 3362.49 & 3382.43 & 3402.48 & 3422.63 & 3442.89 & 3463.24 & 3483.71 & 3504.28 & 3524.95 & 3545.73 \\
\hline 27 & 3566.62 & 3587.62 & 3608.72 & 3629.93 & 3651.25 & 3672.67 & 3694.21 & 3715.86 & 3737.61 & 3759.48 \\
\hline 28 & 3781.46 & 3803.55 & 3825.75 & 3848.07 & 3870.50 & 3893.04 & 3915.70 & 3938.47 & 3961.36 & 3984.36 \\
\hline 29 & 4007.48 & 4030.71 & 4054.06 & 4077.53 & 4101.12 & 4124.83 & 4148.65 & 4172.59 & 4196.66 & 4220.84 \\
\hline 30 & 4245.15 & 4269.58 & 4294.13 & 4318.80 & 4343.60 & 4368.52 & 4393.56 & 4418.73 & 4444.02 & 4469.44 \\
\hline 31 & 44.94 .98 & 4520.65 & 4546.45 & 4572.38 & 4598,43 & 4624.62 & 4650.93 & 467737 & 4703.95 & 4730.65 \\
\hline 32 & 4757.49 & 4784.46 & 4811.56 & 4838.79 & 4866.16 & 4893.66 & 4921.29 & 4949.07 & 4976.97 & 5005.02 \\
\hline 33 & 5033.20 & 5061.52 & 5089.97 & 5118.57 & 5147.30 & 5176.17 & 5205.19 & 5234.34 & 5263.64 & 5293.08 \\
\hline 34 & 5322.66 & 5352.39 & 5382.25 & 5412.27 & 5442.42 & 5472.73 & 5503.18 & 5533.77 & 5564.52 & 5595.41 \\
\hline 35 & 5626.45 & 5657.63 & 5688.97 & 5720.46 & 5752.10 & 5783.89 & 5815.83 & 5847.93 & 5880.18 & 5912.58 \\
\hline 36 & 5945.14 & 5977.85 & 6010.72 & 6043.74 & 6076.92 & 6110.26 & 6143.75 & 6177.41 & 6211.22 & 6245.20 \\
\hline 37 & 6279.33 & 6313.63 & 6348.09 & 6382.71 & 6417.50 & 6452.44 & 6487.56 & 6522.83 & 6558.28 & 6593.89 \\
\hline 38 & 6629.66 & 6665.61 & 6701.72 & 6738.00 & 6774.45 & 6811.08 & 6847.87 & 6884.83 & 6921.97 & 6959.28 \\
\hline 39 & 6996.76 & 7034.42 & 7072.25 & 7110.26 & 7148.44 & 7186.80 & 7225.34 & 7264.06 & 7302.96 & 7342.03 \\
\hline 40 & 7381.29 & 7420.73 & 7460.35 & 7500.15 & 7540.13 & 7580.30 & 7620.65 & 7661.19 & 7701.92 & 7742.83 \\
\hline 41 & 7783.92 & 7825.21 & 7866.69 & 7908.35 & 7950.21 & 7992.25 & 8034.49 & 8076.92 & 8119.54 & 8162.36 \\
\hline 42 & 8205.37 & 8248.57 & 8291.98 & 8335.58 & 8379.37 & 8423.37 & 8467.56 & 8511.95 & 8556.55 & 8601.34 \\
\hline 43 & 8646.34 & 8691.54 & 8736.94 & 8782.55 & 8828.36 & 8874.38 & 8920.60 & 8967.03 & 9013.67 & 9060.52 \\
\hline 44 & 9107.58 & 9154.84 & 9202.32 & 9250.01 & 9297.92 & 9346.03 & 9394.36 & 9442.91 & 9491.67 & 9540.65 \\
\hline 45 & 9589.84 & 9639.25 & 9688.89 & 9738.74 & 9788.81 & 9839.10 & 9889.62 & 9940.36 & 9991.32 & 10042.50 \\
\hline 46 & 10093.92 & 10145.55 & 10197.42 & 10249.51 & 10301.83 & 10354.38 & 10407.16 & 10460.18 & 10513.42 & 10566.90 \\
\hline 47 & 10620.61 & 10674.55 & 10728.73 & 10783.15 & 10837.80 & 10892.69 & 10947.82 & 11003.19 & 11058.79 & 11114.64 \\
\hline 48 & 11170.74 & 11227.07 & 11283.65 & 11340.47 & 11397.54 & 11454.86 & 11512.42 & 11570.23 & 11628.29 & 11686.60 \\
\hline 49 & 11745.15 & 11803.97 & 11863.03 & 11922.35 & 11981.92 & 12041.74 & 12101.83 & 12162.17 & 12222.76 & 12283.62 \\
\hline 50 & 12344.73 & 12406.11 & 12467.75 & 12529.65 & 12591.81 & 12654.24 & 12716.93 & 12779.89 & 12843.11 & 12906.61 \\
\hline 51 & 12970.37 & 13034.40 & 13098.70 & 13163.27 & 13228.12 & 13293.24 & 13358.63 & 13424.30 & 13490.25 & 13556.47 \\
\hline 52 & 13622.97 & 13689.75 & 13756.81 & 13824.15 & 13891.78 & 13959.68 & 14027.87 & 14096.35 & 14165.11 & 14234.16 \\
\hline 53 & 14303.49 & 14373.12 & 14443.03 & 14513.23 & 14583.73 & 14654.52 & 14725.60 & 14796.98 & 14868.65 & 14940.62 \\
\hline 54 & 15012.89 & 15085.45 & 15158.32 & 15231.49 & 15304.96 & 15378.73 & 15452.80 & 15527.18 & 15601.86 & 15676.86 \\
\hline 55 & 15752.16 & 15827.76 & 15903.68 & 15979.91 & 16056.45 & 16133.31 & 16210.47 & 16287.96 & 16365.76 & 16443.87 \\
\hline 56 & 16522.31 & 16601.06 & 16680.13 & 16759.53 & 16839.25 & 16919.29 & 16999.65 & 17080.34 & 17161.36 & 17242.71 \\
\hline 57 & 17324.38 & 17406.39 & 17488.72 & 17571.39 & 17654.39 & 17737.72 & 17821.39 & 17905.40 & 17989.74 & 18074.42 \\
\hline 58 & 18159.44 & 18244.81 & 18330.51 & 18416.56 & 18502.95 & 18589.69 & 18676.77 & 18764.20 & 18851.98 & 18940.11 \\
\hline 59 & 19028.59 & 19117.42 & 19206.60 & 19296.14 & 19386.04 & 19476.29 & 19566.90 & 19657.86 & 19749.19 & 19840.88 \\
\hline
\end{tabular}


TABLE 6. Saturation vapor pressure over water (IPTS-68)-Continued

\begin{tabular}{|c|c|c|c|c|c|c|c|c|c|c|}
\hline $\begin{array}{c}\text { Temperature } \\
\text { deg C }\end{array}$ & 0.0 & 0.1 & 0.2 & 0.3 & 0.4 & 0.5 & 0.6 & 0.7 & 0.8 & 0.9 \\
\hline & pascals & pascals & pascals & pascals & pascals & pascals & pascals & pascals & pascals & pascals \\
\hline 60 & 19932.93 & 20025.34 & 20118.12 & 20211.26 & 20304.77 & 20398.65 & 20492.90 & 20587.52 & 20682.51 & 20777.87 \\
\hline 61 & 20873.61 & 20969.72 & 21066.21 & 21163.07 & 21260.32 & 21357.94 & 21455.95 & 21554.33 & 21653.10 & 21752.26 \\
\hline 62 & 21851.80 & 21951.73 & 22052.04 & 22152.75 & 22253.85 & 22355.33 & 22457.21 & 22559.49 & 22662.16 & 22765.23 \\
\hline 63 & 22868.70 & 22972.56 & 23076.83 & 23181.50 & 23286.57 & 23392.04 & 23497.92 & 23604.21 & 23710.91 & 23818.01 \\
\hline 64 & 23925.53 & 24033.45 & 24141.79 & 24250.55 & 24359.72 & 24469.31 & 24579.31 & 24689.74 & 24800.58 & 24911.85 \\
\hline 65 & 25023.54 & 25135.66 & 25248.20 & 25361.17 & 25474.56 & 25588.39 & 25702.65 & 25817.34 & 25932.46 & 26048.02 \\
\hline 66 & 26164.02 & 26280.45 & 26397.32 & 26514.64 & 26632.39 & 26750.59 & 26869.23 & 26988.32 & 27107.85 & 27227.83 \\
\hline 67 & 27348.27 & 27469.15 & 27590.48 & 27712.27 & 27834.52 & 27957.22 & 28080.38 & 28204.00 & 28328.08 & 28452.62 \\
\hline 68 & 28577.62 & 28703.09 & 28829.03 & 28955.43 & 29082.30 & 29209.64 & 29337.46 & 29465.74 & 29594.50 & 29723.74 \\
\hline 69 & 29853.45 & 29983.65 & 30114.32 & 30245.47 & 30377.11 & 30509.23 & 30641.84 & 30774.93 & 30908.51 & 31042.59 \\
\hline 70 & 31177.15 & 31312.21 & 31447.76 & 31583.81 & 31720.35 & 31857.39 & 31994.94 & 32132.98 & 32271.53 & 32410.58 \\
\hline 71 & 32550.14 & 32690.21 & 32830.78 & 32971.87 & 33113.46 & 33255.57 & 33398.20 & 33541.34 & 33685.00 & 33829.18 \\
\hline 72 & 33973.88 & 34119.10 & 34264.84 & 34411.11 & 34557.91 & 34705.24 & 34853.09 & 35001.48 & 35150.40 & 35299.85 \\
\hline 73 & 35449.84 & 35600.37 & 35751.43 & 35903.04 & 36055.19 & 36207.88 & 36361.12 & 36514.90 & 36669.23 & 36824.11 \\
\hline 74 & 36979.55 & 37135.53 & 37292.07 & 37449.17 & 37606.82 & 37765.03 & 37923.81 & 38083.14 & 38243.04 & 38403.51 \\
\hline 75 & 38564.54 & 38726.14 & 38888.31 & 39051.05 & 39214.37 & 39378.26 & 39542.72 & 39707.77 & 39873.39 & 40039.60 \\
\hline 76 & 40206.39 & 40373.76 & 40541.73 & 40710.27 & 40879.41 & 41049.14 & 41219.46 & 4.1390.38 & 41561.89 & 41734.00 \\
\hline 77 & 41906.71 & 42080.02 & 42253.93 & 42428.45 & 42603.57 & 42779.30 & 42955.64 . & 43132.59 & 43310.16 & 43488.34 \\
\hline 78 & 43667.13 & 43846.54 & 44026.57 & 44207.22 & 44388.50 & 44570.40 & 44752.92 & 44936.08 & 45119.86 & 45304.27 \\
\hline 79 & 45489.32 & 45675.00 & 45861.32 & 46048.28 & 46235.87 & 46424.11 & 46612.99 & 46802.52 & 46992.69 & 47183.51 \\
\hline 80 & 47374.98 & 47567.11 & 47759.89 & 47953.32 & 48147.41 & 48342.16 & 48537.57 & 48733.64 & 48930.38 & 49127.78 \\
\hline 81 & 25.85 & 49524.59 & 49724.00 & 49924.09 & 50124.85 & 50326.28 & 50528.40 & 50731.19 & 50934.67 & 51138.83 \\
\hline 82 & 51343.68 & 51549.22 & 51755.44 & 51962.36 & & 52378.27 & 52587.27 & 52796.97 & 53007.37 & 53218.47 \\
\hline 83 & 53430.28 & 53642.79 & 53856.01 & 54069.94 & 54284.58 & 54499.93 & 54716.00 & 54932.78 & 55150.29 & 55368.51 \\
\hline 84 & 55587.46 & 55807.14 & 56027.53 & 56248.66 & 56470.52 & 56693.11 & 56916.43 & 57140.49 & 57365.29 & 57590.82 \\
\hline 85 & 57817.10 & 58044.12 & 58271.89 & 58500.41 & 58729.67 & 58959.68 & 59190.45 & 59421.97 & 59654.25 & 59887.29 \\
\hline 86 & 60121.09 & & 60590.98 & 60827.07 & 61063.94 & 61301.57 & 61539.97 & 61779.15 & 62019.11 & 62259.84 \\
\hline 87 & 62501.36 & 62743.65 & 62986.73 & 63230.60 & 63475.26 & 63720.70 & 63966.94 & 64213.98 & 64461.80 & 64710.43 \\
\hline 88 & 64959.86 & 65210.09 & 65461.12 & 65712.96 & 65965.61 & 66219.07 & 66473.34 & 66728.43 & 66984.33 & 67241.05 \\
\hline 89 & 67498.60 & 67756.96 & 68016.15 & 68276.16 & 68537.01 & 68798.68 & 69061.19 & 69324.53 & 69588.71 & 69853.73 \\
\hline 90 & & 70386.30 & 70653.85 & 70922.24 & 71191.49 & 71461.58 & & & & 72550.53 \\
\hline 91 & 72824.92 & 73100.17 & 73376.29 & 73653.27 & 73931.13 & 74209.86 & 74489.46 & 74769.94 & 75051.30 & 75333.54 \\
\hline 92 & 75616.66 & 75900.67 & 76185.57 & 76471.36 & 76758. & 77045.61 & 77334.08 & 77623.45 & 77913.72 & 78204.89 \\
\hline 93 & 78496.96 & 78789.95 & 79083.84 & 79378.65 & 79674.36 & 79971.00 & 80268.55 & 80567.02 & 80866.42 & 81166.74 \\
\hline 94 & 81467.99 & 81770.16 & 82073.27 & 82377.31 & 82682.29 & 82988.20 & 83295.06 & 83602.86 & 83911.60 & 84221.29 \\
\hline 95 & 84531.93 & 84843.52 & 85156.07 & 85469.57 & 85784.03 & 86099.44 & 86415.83 & 86733.1 & 87051.49 & 87370.77 \\
\hline 96 & 87691.03 & 88012.26 & 88334.47 & 88657.66 & 88981.82 & 89306.97 & 89633.11 & 89960.24 & 90288. & 90617.46 \\
\hline 97 & 90947.56 & 91278.66 & 91610.76 & 91943. & 92277. & 92613.08 & 92949.20 & 93286.34 & 93624.48 & 93963.65 \\
\hline 98 & 94303.83 & 94645.03 & 94987.26 & 95330.51 & 95674.79 & 96020.09 & 96366.43 & 96713.81 & 97062.22 & 97411.68 \\
\hline 99 & 97762.17 & 98113.71 & 98466.30 & 98819.94 & 99174.62 & 99530.37 & 99887.16 & 100245.02 & 100603.94 & 100963.92 \\
\hline 100 & 324.97 & & & & & & & & & \\
\hline
\end{tabular}

\section{Discussion}

\subsection{Thermodynamic Temperature}

IPTS-68 was adopted as a replacement for IPTS-48 on the basis, at least in part, that it approached the thermodynamic temperature scale to within the uncertainty of the latter. Presumably, therefore, the conversion of $\gamma$ and of $B^{\prime}$ and $C^{\prime}$ from IPTS-48 to IPTS68 , and the substitution of these quantities into eqs (10) and (11) should yield a solution giving vapor pressure on IPTS-68. This is not the case. The conversion of $\gamma$ from IPTS-48 to IPTS-68 introduces a maximum change in $\gamma$, which occurs at $50{ }^{\circ} \mathrm{C}$, of about 0.04 joules per gram, a quantity which has negligible effect on $p$. Similarly, $B^{\prime}$ and $C^{\prime}$, and consequently $p$, are affected insignificantly by the change in temperature scales. The lower limits of integration, $p_{0}$ and $T_{0}$, remain identical on the two scales. Thus, when temperature scale changes in $\gamma, B^{\prime}$ and $C^{\prime}$ are made, eq (10) still leads to a formulation that yields vapor pressures in substantial agreement with Stimson's vapor pressures on IPTS -48 and not on IPTS-68. The inference from these results is that IPTS-48 may be a closer approximation to the thermodynamic temperature scale in the range 0 to $100{ }^{\circ} \mathrm{C}$ than IPTS-68. It is for this reason that we based our derivation on IPTS-48 rather than IPTS-68.

\subsection{Error Analysis}

It is of interest to estimate the uncertainty in calculating $p$ arising from estimated errors in $\gamma, Z, R, T$, and $T_{0}$, using the Clausius-Clapeyron equation. For purposes of this error analysis, we shall derive a simpli- 
fied vapor pressure equation and we shall assume that all variables are independent. Equation (5) serves as a convenient starting point for this analysis:

$$
\frac{d p}{p}=\frac{\gamma}{R T^{2} Z} d T
$$

The calorimetric quantity $\gamma$ varies from 2500 to 2257 $\mathrm{j} / \mathrm{g}$ as $t$ increases from 0 to $100^{\circ} \mathrm{C}$. As an approximation let $\gamma$ be equal to $2400 \mathrm{j} / \mathrm{g}$ and constant. The compressibility factor $Z$ varies from 0.9995 to 0.9845 over the same temperature range. Let $Z$ be approximated by unity. Under these conditions, the integration of eq (5) yields

or

$$
\ln \frac{p}{p_{0}}=-\left[\frac{\gamma}{R T Z}\right]_{T_{0}}^{T}
$$

$$
p=p_{o} e^{-\frac{\gamma}{R T_{0} Z}\left[\frac{T_{0}}{T}-1\right] .}
$$

Differentiating $p$ with respect to each parameter, replacing the differentials by finite increments, and dividing each side by $p$, we obtain

$$
\begin{aligned}
& \frac{\Delta p}{p}=-\frac{1}{R T_{0} Z}\left[\frac{T_{0}}{T}-1\right] \Delta \gamma \\
& \frac{\Delta p}{p}=\frac{\gamma}{R^{2} T_{0} Z}\left[\frac{T_{0}}{T}-1\right] \Delta R \\
& \frac{\Delta p}{p}=\frac{\gamma}{R T_{0} Z^{2}}\left[\frac{T_{0}}{T}-1\right] \Delta Z \\
& \frac{\Delta p}{p}=\frac{\gamma}{R T^{2} Z} \Delta T \\
& \frac{\Delta p}{p}=-\frac{\gamma}{R T_{0}^{2} Z} \Delta T_{0} .
\end{aligned}
$$

If we let the finite increments represent estimated errors in their respective parameters, then these equations permit us to calculate the corresponding estimated relative error in pressure $\frac{\Delta p}{p}$.

Since $p_{0}$ and $T_{0}$ are the assigned steam point values of pressure and temperature on IPTS-48, and since our derivation was made on this scale, it will be assumed that both $p_{0}$ and $T_{0}$ have zero error. The estimated error in $\gamma$ will be taken as $0.23 \mathrm{j} / \mathrm{g}$, the residual standard deviation of the least square fit of eq (8) to the Osborne, Stimson, and Ginnings data. The estimated error in $R$, the specific gas constant for water vapor, arises from the estimated errors in the universal molar gas constant and in the molecular weight of water. According to Bain [44], the error limit in $R$, defined as three times the standard deviation, is $7 \times 10^{-5} \mathrm{j} / \mathrm{gK}$. We will use the standard deviation as our estimate of error in $R$, i.e., $2.3 \times 10^{-5} \mathrm{j} / \mathrm{gK}$. Goff [19] has assigned tolerances to the Goff and Gratch virial coefficients which he set at twice the probable error. We have converted his tolerances to standard devia- tions, and computed, as the equivalent estimated error in $Z$, the quantity $2 \times 10^{-4}$, valid from 0 to $100{ }^{\circ} \mathrm{C}$. The absolute temperature $T$ enters into eq (26) as the independent variable so that it is subject neither to experimental nor scale error. Experimental and scale errors in $T$ affect $\gamma$ and the virial coefficients; however, these temperature errors show up and are contained in the assigned estimated errors in $\gamma$ and $Z$.

A summary of the relative errors in $p$ due to the estimated errors in $\gamma, R$, and $Z$ is given in table 7 . Assuming that the estimated errors in $\gamma, R$, and $Z$

\begin{tabular}{|c|c|c|c|c|c|}
\hline \multirow{3}{*}{$\begin{array}{c}\text { Tempera- } \\
\text { ture } \\
t \\
{ }^{\circ} \mathrm{C}\end{array}$} & \multicolumn{3}{|c|}{$\begin{array}{l}\text { Estimated relative error in } \\
\text { vapor pressure due to } \\
\text { estimated uncertainties in }\end{array}$} & \multirow{2}{*}{\multicolumn{2}{|c|}{$\begin{array}{c}\text { Estimated maximum } \\
\text { error in } \\
\text { vapor pressure }\end{array}$}} \\
\hline & \multirow{2}{*}{$\gamma$} & \multirow{2}{*}{$\begin{array}{c}R \\
p p m\end{array}$} & \multirow{2}{*}{$\begin{array}{c}Z \\
p p m\end{array}$} & & \\
\hline & & & & ppm & pascals \\
\hline 0 & 490 & 254 & 1021 & 1765 & 1.08 \\
\hline 10 & 426 & 221 & 887 & 1534 & 1.88 \\
\hline 20 & 366 & 190 & 761 & 1317 & 3.08 \\
\hline 30 & 310 & 161 & 644 & 1115 & 4.73 \\
\hline 40 & 257 & 133 & 535 & 925 & 6.82 \\
\hline 50 & 208 & 108 & 432 & 748 & 9.23 \\
\hline 60 & 161 & 83 & 335 & 579 & 11.54 \\
\hline 70 & 117 & 61 & 244 & 422 & 13.15 \\
\hline 80 & 76 & 39 & 158 & 273 & 12.93 \\
\hline 90 & 37 & 19 & 77 & 133 & 9.32 \\
\hline 100 & 0 & 0 & 0 & 0 & 0 \\
\hline
\end{tabular}
are systematic, then the total estimated error in $p$ is at most the arithmetic sum of the estimated errors in $p$ due to $\Delta \gamma, \Delta R$, and $\Delta Z$, respectively. This error analysis sets the outer bounds, that is, the estimated maximum error, that might occur in the calculation of $p$.

\section{TABLE 7. Estimated uncertainty in thermodynamic} computation of vapor pressure

The deviations of our formulation from Stimson's data may be taken as a measure of the actual error in the calculation of vapor pressure. Therefore, one would expect these deviations to be comparable to the estimated maximum errors in the calculation of $p$, but they are two orders of magnitude smaller. It is possible that the estimates of uncertainty assigned to $\gamma, R$, and $Z$ may be conservative leading to estimated maximum errors in $p$ that are too large. It is also possible that the systematic errors in $\gamma, R$, and $Z$ tend to compensate each other whereas we assumed that these are additive. Finally, the Goff and Gratch virial coefficients, which we used to compute $Z$, are not completely independent parameters. Goff and Gratch derived these virial coefficients using vapor pressure data, first in computing enthalpy coefficients from calorimetric data, that is, from $\gamma$, and second, in evaluating the constants of integration that result from integrating enthalpy coefficients to obtain virial coefficients. The effect of this procedure is that the virial coefficients are partially dependent on, and selfconsistent with, $p$ and $\gamma$. Although the Cragoe-Stimson measurements were available to Goff and Gratch [6], apparently these were used only for comparison with 
their vapor pressure equation and not for deriving virial coefficients. On the other hand, the Gerry equation [7], the Osborne and Meyers formulation [31] and the Moser and Zmaczynski vapor pressure measurements [37] were used directly or indirectly.

We have employed virial coefficients due to Keyes [56] and to Czuha as given by Bain [44] and obtained equations which predict vapor pressures significantly different from Stimson's measurements, but well within our estimated maximum error. We ascribe, in part, the close concordance of our formulation with Stimson's measurements to our use of the Goff and Gratch virial coefficients.

It is clear that if the parameters $\gamma, R$, and $Z$ were completely independent, then they must be known to an accuracy that is 1 to 2 orders of magnitude better than they are now known if thermodynamic calculations of vapor pressure are to have an uncertainty comparable to Stimson's measurements. Therefore, our formulation should not be taken as a corroboration from completely independent thermodynamic data of Stimson's vapor pressure measurements; rather it should be viewed as a smoothing function that has a theoretical basis. This is particularly true of eq (17) which was adjusted to fit Stimson's measurements. However, it is evident that there is a high level of thermodynamic consistency among the parameters $p, \gamma$, and $Z$.

\section{Conclusions}

The feasibility of computing the vapor pressure of water to a high order of accuracy by a smoothing function that has a rational basis has been demonstrated. Calculated vapor pressures agree with the very precise measurements of Stimson to within $7 \mathrm{ppm}$ from 25 to $100{ }^{\circ} \mathrm{C}$.

At the ice point the PTR data is in reasonable accord with the value calculated from eq (17); at the triple point and ice point, steam table values are very close to our calculated values. Prytz's triple point measurement is 0.3 pascal $(490 \mathrm{ppm})$ higher than our calculated value.

It should be noted that the triple point, the most stable condition of the pure water substance, which serves as a defining fixed point on the thermodynamic temperature scale and is reproducible in temperature to a very high order of accuracy, has a comparatively inexactly known saturation pressure. A definitive determination of the vapor pressure at the triple point is needed.

Douslin's measurements from 0 to $20{ }^{\circ} \mathrm{C}$ are systematically higher than our calculations by 0.6 to 1.7 pascals, roughly of the order of 2 to 3 times his estimated maximum systematic error.

The Moser and Zmaczynski data from 73 to $100{ }^{\circ} \mathrm{C}$ are systematically lower than the values predicted by eq (17) whereas the Beattie and Blaisdell data, which only encompass the range 96 to $103{ }^{\circ} \mathrm{C}$, deviate randomly about our curve with an estimated residual standard deviation of 1.55 pascals (17 ppm).

Equation (17) has seven terms, and where a simpler equation is desired, eq (18b) with five terms can be used with little degradation in the accuracy of the computed values. Equation (24) with $n=3$ on IPTS -68 is equivalent to eq (18b) on IPTS -48 .

\section{References}

[1] Goff, J. A., Saturation pressure of water on the new kelvin scale, Humidity and Moisture, Arnold Wexler, Editor-inchief, vol. 3 (Reinhold Publishing Corporation, New York, 1965) p. 289

[2] Stimson, H. F., Some precise measurements of the vapor pressure of water in the range from 25 to $100{ }^{\circ} \mathrm{C}$, J. Res. Nat. Bur. Stand. (U.S.), 73A (Phys. and Chem.) No. 4, 493-496 (Sept.-Oct. 1969).

[3] Stimson, H. F., Precision resistance thermometry and fixed points. Temperature, Its Measurement and Control in Science and Industry, Vol. 2, American Institute of Physics Symposium, 1939 (New York City, Reinhold Publishing Co.)

[4] Osborne, Nathan S., Calorimetry of a fluid, J. Res. Nat. Bur. Stand. (U.S.), 4, 609-629 (1930)RP168.

[5] Goff, J. A. and Gratch, S., Thermodynamic properties of moist air. Heating, Piping and Air Conditioning, ASHVE Journal Section 17, 334 (1945).

[6] Goff, John A. and Gratch, S., Low-pressure properties of water from -160 to $212 \mathrm{~F}$, Trans. ASHVE 52, 95 (1946).

[7] Osborne, N. S., Stimson, H. F., and Ginnings, D. C., Measurements of heat capacity and heat of vaporization of water in the range $0^{\circ}$ to $100{ }^{\circ} \mathrm{C}$, J. Res. Nat. Bur. Stand. (U.S.), 23, 197-260 (1939)RP1228.

[8] Fiock, E. F. and Ginnings, D. C., Heat of vaporization of water at $50^{\circ}, 70^{\circ}$, and $90^{\circ} \mathrm{C}, \mathrm{J}$. Res. Nat. Bur. Stand. (U.S.), 8, 321324 (1932)RP416.

[9] Osborne, N. S., Stimson, H. F., and Ginnings, D. C., Calorimetric determination of the thermodynamic properties of saturated water in both the liquid and gaseous states from $100^{\circ}$ to $374{ }^{\circ} \mathrm{C}$, J. Res. Nat. Bur. Stand. (U.S.), 18, 389-447 (1937)RP983.

[10] Burgess, G. K., The international temperature scale, J. Res. Nat. Bur. Stand. (U.S.), 1, 635-640 (1928)RP22.

[11] Stimson, H. F., International temperature scale of 1948, J. Res. Nat. Bur. Stand. (U.S.), 42, 209-217 (1949)RP1962.

[12] Stimson, H. F., International practical temperature scale of 1948. Text revision of 1960 , J. Res. Nat. Bur. Stand. (U.S.), 65A (Phys. and Chem.), No. 3, 139-145 (May-June 1961)

[13] Keyes, F. G., Smith, L. B., and Gerry, H. T., The specific volume of steam in the saturated and superheated condition together with derived values of the enthalpy, entropy, heat capacity and Joule Thompson coefficients. Proc. Am. Acad. Arts Sci. 70, 319 (1936).

[14] Keyes, F. G., The consistency of the thermodynamic data for water substance vapor phase to $550{ }^{\circ} \mathrm{C}, \mathrm{J}$. Chem. Phys. 17 , 923 (1949).

[15] Keyes, F. G., The 1957 status of steam properties, Trans. ASME 80, 555 (1958).

[16] Keenan, J. H., Keyes, F. G., Hill, P. G., and Moore, J. G., Steam Tables (John Wiley and Sons, Inc., New York, 1969).

[17] Knoblauch, O., Linde, R. and Klebe H., Die thermischen Eigenshaften des gesättigen und des überhitzten Wasserdampfes zwischen $100^{\circ}$ und $180^{\circ} \mathrm{C}$, Mittheilung über Forschungsarbeiten d. Ver. d. Ing. 21, 33 (1905).

[18] Collins, S. C., and Keyes, F. G., The heat capacity and pressure variation of the enthalpy for steam from $38^{\circ}$ to $125^{\circ} \mathrm{C}$. Proc. Am. Acad. Arts. Sci. 72, 283 (1938).

[19] Goff, J. A., Standardization of thermodynamic properties of moist air-final report of the Working Subcommittee, International Joint Committee on Psychrometric Data, ASHVE J. Section, Heating, Piping and Air Conditioning 55, 118 (1949).

[20] New values for the physical constants, Nat. Bur. Stand. (U.S.), Tech. News Bull. 47 (10) 175 (1963).

[21] ASTM Metric Practice Guide, Nat. Bur. Stand. (U.S.), Handb. 102, 49 pages (1967).

[22] Abramowitz, M., and Stegun, I. A., Handbook of Mathematical Functions, Nat. Bur. Stand. (U.S.), Appl. Math. Ser. 55, 885 (June 1964). 
[23] Partington, J. R., An Advanced Treastise on Physical Chemistry, vol. 2 (Longman, London, 1951).

[24] Miller, D. G., Estimating vapor pressures - a comparison of equations, Ind. Eng. Chem. 56, 46 (1964).

[25] Ambrose, D., Councell, J. F., and Davenport, A. J., The use of Chebyshev polynomials for the representation of vapour pressures between the triple point and the critical point. J. Chem. Thermo. 2, 283 (1970).

[26] Goodwin, R. D., Nonanalytic vapor pressure equation with data for nitrogen and oxygen. J. Res. Nat. Bur. Stand. (U.S.), 73A, (Phys. and Chem.) No. 5, 487-491 (Sept.-Oct. 1969).

[27] Holborn, L., and Henning, F. Über das Platinthermometer und den Sättigungsdruck des Wasserdampfes zwischen 50 and $200^{\circ}$, Ann. Physik. IV, 26, 54 (1908).

[28] Scheel, K., and Heuse, W. Bestimmung des Sättigungsdruckes von Wasserdampf zwischen 0 und $+50^{\circ}$, Ann. Physik. IV 31, 715 (1910).

[29] Holborn, L., Scheel, K., and Henning, F., Wärmetabellen (Vieweg and Sohn, 1919).

[30] Smith, L. B., Keyes, F. G., and Gerry, H. T., The vapor pressure of water, Proc. Am. Acad. Arts and Sci.69, 137 (1934).

[31] Osborne, N. S., and Meyers, C. H., A formula and tables for the pressure of saturated water vapor in the range 0 to $374{ }^{\circ} \mathrm{C}$, J. Res. Nat. Bur. Stand. (U.S.), 13, 1-20 (1934)RP691.

[32] The International Practical Temperature Scale of 1968, Metrologia 5, 35 (1969).

[33] Volet, Ch., La température d'ébullition d'eau d'après les experiences de P. Chappuis, Travaux et Mémoires du Bureau international des Poids et Measures 18, 11 (1929).

[34] Zmaczynski, A., and Bonhoure, A., La température d'ébullition de l'eau en fonction de la pression, J. Phys. et Rad. (VII) 1, 285, (1930).

[35] Moser, H., Eine statische Methode für präzise Dampfdruckmessungen bei höheren Temperaturen und ihre Anwendung zur Sicherung des Hundertpunktes der Temperaturskala, Ann. Phys. (5) 14, 790 (1932).

[36] Beattie, J. A. and Blaisdell, B. E., An experimental study of the absolute temperature scale. III. The reproducibility of the steam point. The effect of pressure on the steam point, Proc. Am. Acad. Arts Sci. 71, 361 (1937).

[37] Moser, H. and Zmaczynski, A. Sättigungsdruck von Wasserdampf Zwischen 73 und $130^{\circ}$, Physik. Zeits 40, 221 (1939).

[38] Michels, A., Blaisse, B., Ten Seldam, C. A. and Wouters, H. M., The vapour pressure of water in the neighborhood of $100{ }^{\circ} \mathrm{C}$. Physica 10,613 (1943).

[39] Douslin, D. R. and McCullough, J. P., An inclined-piston deadweight pressure gage, U.S. Bureau of Mines Rept. of Investigation No. 6149 (1963).

[40] Douslin, D. R., Vapor pressure of water from -2.5 to $20{ }^{\circ} \mathrm{C}$, J. Chem. Thermodynamics, in press.
[41] Thiesen, M. and Scheel, K., Bestimmung der Spannkraft des gesättigen Wasserdampfes bei Temperaturen zwischen $-12^{\circ}$ und $+25^{\circ}$, Wiss. Abh. physik-techn. Reichsanst. 3, $71(1900)$.

[42] Scheel, K. and Heuse, W., Bestimmung des Sättigungsdruckes von Wasserdampf unter $0^{\circ}$, Ann. Physik. 29, 729 (1909).

[43] Prytz, K., Manomètre a contact optique et son emploi pour la determination du triple point d'eau, Mathematisk-fysike meddelelser Danske Videnskabernes Selskab, Copenhagen 1 1, No. 2(1931).

[44] Bain, R. W., N.E.L. Steam Tables, 1964, Her Majesty's Stationery Office, Edinburgh, 1964.

[45] Sonntag, D., Hygrometrie, pp. 91-102 (Akademie-Verlag, Berlin, 1966-1968).

[46] Osborne, N. S., Stimson, H. F. and Ginnings, D. C., Thermal properties of saturated water and steam, J. Res. Nat. Bur. Stand. (U.S.), 23, 261-270 (1939) RP1229.

[47] APIRP 44 Table 2-1-(1.01)-k, American Petroleum Institute Research Project 44, Texas A and M University, Chemical Thermodynamics Properties Center, Dept. of Chem., College Station, Texas (1964).

[48] Meyer, C. A., McClintock, R. B., Silvestri, G. J., and Spencer, Jr., R. C. 1967 ASME Steam Tables, American Soc. Mech. Eng., New York.

[49] Bridgeman, O. C., and Aldrich, E. W., Vapor pressure tables for water, Trans ASME. J. Heat Transfer Series C-D 86, 279 (1964).

[50] Gibson, M. R. and Bruges, E. A., New equations for the thermodynamic properties of saturated water in both the liquid and vapor phases, J. Mech. Eng. Science 9, 24 (1967).

[51] The Third International Conference on Steam Tables, Mech. Eng. 57, 710 (1935)

[52] ASHRAE Guide and Data Book, Fundamentals and Equipment. 1961, 1965 and 1966, American Society of Heating Refrigerating and Air Conditioning Engineers, Inc., New York.

[53] List, R. J., Smithsonian Meteorological Tables, Sixth revised edition, Smithsonian Institution, W ashington (1951).

[54] Resolution 19, IV Congress, World Meteorological Organization, 1963.

[55] Douglas, T. B. Conversion of existing calorimetrically determined thermodynamic properties to the basis of the International Practical Temperature Scale of 1968. J. Res. Nat. Bur. Stand. (U.S.), 73A (Phys. and Chem), No. 5, 451-470 (Sept.-Oct. 1969).

[56] Keyes, F. G. The thermodynamic properties of water substance 0 to $150{ }^{\circ} \mathrm{C}$. J. Chem. Phys. 15, 602 (1947).

(Paper 75A3-665) 


\section{Publications of the National Bureau of Standards*}

\section{Selected Abstracts}

Aquista, N., Schoen, L. J., Matrix isolation spectrum of the SH radical, J. Chem. Phys. 53, No. 3, 1290-1291 (August 1, 1970).

Key words: Infrared spectrum; isolated $\mathrm{SH}$; low temperature; matrix; $\mathrm{SH}$; ultraviolet.

The free radical $\mathrm{SH}$ is produced in sufficient quantities by photodeposition of $\mathrm{H}_{2} \mathrm{~S}$ in argon matrices at $20 \mathrm{~K}$ for observation in the ultraviolet and infrared. Close agreement between the matrix data and the gas-phase work in the electronic region support the assignment.

Adler, I., Walter, L. S., Lowman, P. D., Glass, B. P., French, B. M., Philpotts, J. A., Heinrich, K. F. J., Goldstein, J. I., Electron microprobe analysis of Apollo 11 lunar samples (Proc. Apollo 11 Lunar Science Conf., Houston, Texas), Geochim. Cosmochim. 34, Suppl. 1, 87-92 (January 30, 1970).

Key words: Analysis; electron probe; lunar samples; microanalysis; minerals; rocks.

Plagioclase feldspar, clinopyroxene and ilmenite in a polished thin section of a "Type A" crystalline rock were analyzed. The clinopyroxene grains are compositionally variable and both high $\mathrm{Ca}$ and low $\mathrm{Ca}$ phases are present. The plagioclase is compositionally homogeneous. The ilmenite is chemically homogeneous except for occasional, small areas of high local chromium concentration. Accessory minerals are: apatite (containing Cl, F, Y, and Ce), troilite and metallic iron.

Glassy spherules from the lunar soil are mostly similar in composition to the crystalline rocks; however, some appear to have been monomineralic.

The crystalline rock has apparently formed by relatively rapid cooling of a silicate melt under conditions of low partial pressure. Many components of the soil appear to have formed by meteoritic impact.

Anderson, H. J., Brenner, A., Chemical vapor deposition of rhenium, Proc. 2nd Intern. Conf. on Chemical Vapor Deposition, Los Angeles, Calif., May 1970, pp. 356-366 (1970).

Key words: Chemical vapor deposition; rhenium; rhenium coatings; rhenium oxychlorides.

The chemical vapor deposition (CVD) of rhenium from a variety of compounds was investigated. The reduction of perrhenyl chloride and rhenium oxytetrachloride with hydrogen resulted in the production of deposits which ranged from bright to powdery, the latter being obtained at a temperature above $600{ }^{\circ} \mathrm{C}$. The bright deposits were cracked and exfoliated from the substrate. The only ductile deposits were obtained by thermal decomposition of $\mathrm{ReOCl}_{4}$ at about $1200{ }^{\circ} \mathrm{C}$, which process has been previously described by Russian workers.

Ausloos, P., Ion-molecule reactions and photoionization of hydrocarbons, Chapter in Progress in Reaction Kinetics, G. Porter, Ed., 5, 113-179 (March-April 1970).

Key words: Hydrocarbons; ion-molecule reactions; radiolysis and photoionization; rate constants; theory.
This is a critical review of the literature on ion-molecule reactions in the radiolysis and photoionization of hydrocarbons.

Ausloos, P., Lias, S. G., Carbonium ions in radiation chemistry. Reactions of $t$-butyl ions with hydrocarbons, $J$. Am. Chem. Soc. 92, No. 17, 5037-5045 (August 26, 1970).

Key words: Carbonium ions; ion-molecule reactions; neopentane; radiolysis; rate constants; $t$-butyl ion.

$t$-Butyl ions, generated by the $\gamma$-irradiation of neopentane, undergo hydride transfer reactions with alkanes having tertiary hydrogen atoms

$$
t-\mathrm{C}_{4} \mathrm{H}_{9}^{+}+\mathrm{RH} \rightarrow \text { iso }-\mathrm{C}_{4} \mathrm{H}_{10}+\mathrm{R}^{+}
$$

to form isobutane and a tertiary carbonium ion as products. By irradiating neopentane in the presence of mixtures of two reactant molecules, one deuterated and one non-deuterated, the relative rates of the hydride transfer reaction for twenty-two $\mathrm{C}_{5}-\mathrm{C}_{8}$ alkanes were determined with an unusually high accuracy from the observed ratios of iso- $\mathrm{C}_{4} \mathrm{H}_{9} \mathrm{D}$ to iso- $\mathrm{C}_{4} \mathrm{H}_{10}$. Absolute rate constants for the reactions, based on a determination of the rate of depletion of the isobutane yield when ammonia is added, are given. The rate constants are generally very low $\left(10^{-11}\right.$ to $10^{-10} \mathrm{~cm}^{3} /$ molecule-s $)$, but are shown to depend on the heat of reaction, which in turn, depends on the structure of the tertiary product ion, $\mathrm{R}^{+}$. The evidence indicates that the hydride transfer reactions of the $t$-butyl ion have an activation energy, $E_{\text {act }} \leqslant 0.12 \mathrm{eV}$.

Branscomb, L. M., A fully integrated partnership between government laboratory and university, Proc. Education on Federal Laboratory-University Relationships Symp., sponsored by the Federal Council for Science and Technology and the American Council on Education, Washington, D.C., October 29-31, 1968, pp. 139-151 (May 1969).

Key words: Management principles; research management principles.

The pattern of government laboratory-university collaboration inherent in the Joint Institution for Laboratory Astrophysics (JILA) is described, with emphasis on its advantages and shortcomings and the relevant management principles.

Branscomb, L. M., Discussion, Can. J. Chem. 47, No. 10, 17031941 (May 15, 1969).

Key words: Aeronomy; editorial comments.

This manuscript contains the edited comments on invited papers by A. Dalgarno, A. V. Phelps, and E. E. Ferguson which will appear in Transactions of the IAGA (International Association of Geomagnetism and Aeronomy) Symposium on Laboratory Measurements of Aeronomic Interest, September 3-4, 1968, York University, Toronto, Canada. The Transactions are to be published in the Canadian Journal of Chemistry.

Brauer, G. M., Huget, E. F., Termini, D. J., Plastic modified $o$-Ethoxybenzoic acid cements as temporary restorative materials, J. Dental Res. 49, No. 6, 1487-1494 (November-December 1970).

Key words: Dental restorative materials; EBA cements; plastic reinforced restoratives; restorative materials; temporary dental cements. 
To improve the stress-bearing characteristics, powdered polymers of relatively low elastic moduli were incorporated into the powder component of EBA cements. Acrylic and vinyl copolymers appeared to be the most suitable additives for achieving reinforcement. The resultant products exhibited significant increases in tensile strength. These materials possess excellent manipulative qualities. Limited clinical evaluation indicates that some of the polymer-reinforced materials, on the basis of their demonstrated mechanical and palliative properties, appear to be highly useful as long duration temporary restoratives.

Brenner, A., Anderson, H. J., Chemical vapor deposition of rhenium, Proc. Interfinish 1968 7th International Metal Finishing Conf., May 5-9, 1968, Hanover, Germany, pp. 28-31 (1968).

Key words: Carbonyl; chemical vapor deposition; electrodeposition; hexafluoroacetylacetone; rhenium coatings; trifluorophosphine.

Methods of áepositing rhenium coatings are reviewed, and a brief account is given of work now in progress at NBS on the deposition of rhenium. The electrodeposition of rhenium from an aqueous perrhenate bath does not yield a satisfactory coating; work at NBS with various rhenium compounds in both aqueous and nonaqueous solutions did not lead to a better plating process. The chemical vapor deposition (CVD) of rhenium is more promising, particularly the reduction of rhenium hexafluoride with hydrogen. At NBS work has been done with the CVD of rhenium from the carbonyl, the hexafluoroacetylacetone derivative, and work is in progress with the trifluorophosphine derivatives.

Bridges, J. M., Wiese, W. L., Transition probabilities for the prominent red lines of Ne I, Phys. Rev. A. 2, No. 2, 285-293 (August 1970).

Key words: Neon; spectrum; transition probabilities.

Relative transition probabilities of all 30 lines belonging to the prominent $3 s-3 p$ transition array of Ne I have been measured in emission with a wall-stabilized arc operating in an argon-neon mixture atmospheric pressure. The data have been normalized to an absolute scale provided by recent lifetime measurements of the $3 p$-levels. Our results as well as the data from other emission measurements have been subjected to extensive comparisons and tests, including a check for fullfillment of the $J$-file sum rule. It is found that our data exhibit a much better consistency than any other set of individual values. On the basis of this as well as our uncertainty estimates we estimate that the accuracy of our individual transition probabilities for this transition array, except for a few weak lines, is of the order of $10 \%$ on an absolute basis.

Brinckman, F. E., Wachtman, J. B., Jr., Editors, Research materials developed under the NBS inorganic materials program, Nat. Bur. Stand. (U.S.), Spec. Publ. 333, 71 pages (September 1970) 70 cents, SD Catalog No. C13.10:333.

Key words: Chemical properties; composition; gases; glasses; liquids; multiphase materials; physical properties; polycrystals; preparation; research.

The National Bureau of Standards develops many specialized materials in the process of carrying out research supporting its measurements, standards, and service activities. These materials include gases, liquids, glasses, single crystals, polycrystals, and various multiphase materials; their compositions (including trace elements in some cases) and physical characteristics are tailored to specific research needs, but the materials are often of use for other research purposes. Production is usually limited to immediate internal needs and samples are not generally available for distribution, but knowledge of production techniques and materials characteristics which may be helpful to other scientists is available. The present listing of research materials developed in the Inorganic Materials Division accordingly gives names of scientific staff members who may be contacted for this type of information as well as giving a brief summary of the nature, method of preparation and properties determined for each material.

Brown, D. W., Lowry, R. E., Wall, L. A., Radiation-induced copolymerization of tetrafluoroethylene and $3,3,4,4,5,5,5$ - heptafluoropentene-1 under pressure, J. Polymer Sci. Part A-1, 8, No. 9, 2441-2452 (1970).

Key words: Amorphous polymer; copolymerization; fluoropolymers; glass temperature; $3,3,4,4,5,5,5$-heptafluoropentene-1; high pressure; radiation-induced; tetrafluoroethylene.

An investigation was made of the gamma-ray induced copolymerization of tetrafluoroethylene and 3,3,4,4,5,5,5-heptafluoropentene- 1 . At $22{ }^{\circ} \mathrm{C}$ and 5000 and 10,000 atm the polymerization rate changes little between 0 and 75 mole percent tetrafluoroethylene. Above 90 mole percent the rate increases greatly. Molecular weights vary in a fashion similar to the variation in the rates. Between 10,000 and $15,000 \mathrm{~atm}$ the viscosity of the pentene increases by a factor of $10^{5}$. A rate maximum occurs in this pressure range; polymerization continues long after irradiation is ended. Both reactivity ratios favor the pentene.

Several copolymer properties were studied. The polymers are amorphous and soluble in perfluoro-ethers, -alkanes, and -aromatics if they contain less than $80 \%$ tetrafluoroethylene. The glass temperatures of the amorphous polymers decrease and the thermal and radiation stability increases as the tetrafluoroethylene content increases.

Carrington, T., Garvin, D., The chemical production of excited states, Chapter 3 in Comprehensive Chemical Kinetics Volume 3. The Formation and Decay of Excited Species, pp. 107-181 (1969).

Key words: Atom-transfer reaction; chemi-excitation; chemical activation; chemical kinetics; gas phase; potential energy surfaces; recombination; review; unimolecular decomposition.

Chemical excitation, the production of molecules in non-equilibrium population distributions in electronic, vibrational and rotational states is described.

The applicable parts of reaction rate theory are reviewed. Typical cases are used as examples of chemi-excitation in combination, group transfer and decompostion reactions. Reactions involving excited electronic states are discussed in terms of the interaction of potential surfaces. Applications to lasers and to the study of unimolecular decompositions are described.

Cezairliyan, A., McClure, J. L., Morse, M. S., Beckett, C. W., Measurement of heat capacity of tantalum in the range 1900 $3000 \mathrm{~K}$ by a pulse heating method, Proc. 5th Symp. Thermophysical Properties, Newton, Mass., September 30-October 2, 1960 , C. F. Bonilla, Ed., pp. 385-390 (American Society of Mechanical Engineers, New York, N.Y., 1970).

Key words: Heat capacity; high-speed measurements; high temperature; tantalum; thermodynamics.

A technique is described for the high-speed (millisecond resolution) measurement of heat capacity of electrical conductors at high temperatures (above $1900 \mathrm{~K}$ ). Measurements on heat capacity of tantalum in the temperature range 1900 to $3000 \mathrm{~K}$ are reported and are compared with those in the literature. Duration of an individual experiment in which the specimen was heated from room temperature to close to its melting point is less than one second. Temperature measurements were made with a high-speed photoelectric pyrometer. Experimental quantities (voltage, current, temperature) were recorded with a high-speed digital data acquisition system which has a time resolution of $0.4 \mathrm{~ms}$. Estimated inaccuracy of heat capacity measurements in the above temperature range is 2 to 3 percent.

Cezairliyan, A., Morse, M. S., Berman, H. A., Improvements in oscilloscopic measurements in high-speed experiments, Nat. Bur. Stand. (U.S.), Tech. Note 539, 20 pages (Oct. 1970) 30 cents, SD Catalog No. C13.46:539.

Key words: High-speed measurements; high-speed recording; oscilloscopes.

Two refinements in oscilloscopic recording have been made which improve considerably the recording of isolated events in heavy 
current discharge studies, where substantially rectangular pulses are employed. The accuracy of the method employed has been verified experimentally to be in the region of 0.01 to 0.1 percent. The first refinement is a unit for the differential suppression of the incoming signal by an adjustable amount, and the second refinement is a system by which time markers are sent to several oscilloscopes at adjustable time intervals simultaneously with the actual incoming signal.

Chandler, H. H., Bowen, R. L., Paffenbarger, G. C., Mullineaux, A. L., Clinical investigation of a radiopaque composite restorative material, $J$. Am. Dental Assoc. 81, No. 4, 935-940 (October 1970).

Key words: Clinical research (dental); composite restorations; diagnosis; physical properties of fillings; $x$-ray opaque.

A radiopaque composite restorative material was developed using a ternary eutectic dimethacrylate liquid formulation as the binder and $\mathrm{a} \mathrm{BaF}_{2}$-containing glass and fused silica as the reinforcing fillers. One hundred and ten restorations were placed using this newly developed material. The restorations will be observed over a prolonged period. They had sufficient radiopacity and yet were esthetically pleasing. Postoperative radiographs showed comparatively radiolucent areas in and around many of the restorations. These radiolucencies were usually caused by unfilled spaces at the tooth-restoration interface.

Christ, B. W., Smith, G. V., Effects of nitrogen in lattice solution on the yielding and flow of zone-refined iron polycrystals between 128 and $300 \mathrm{~K}$, Trans. Met. Soc. AIME 1, No. 4, 827-833 (April 1970).

Key words: Flow stress; impurity softening; lattice solution; lower yield stress; nitrogen; polycrystals; solution hardening; upper yield point; zone-refined iron.

$\mathrm{Up}$ to 0.18 at. pct $\mathrm{N}$ was introduced into lattice solution in hydrogenpurfied, zone-refined iron polycrystals. Tensile tests were conducted at $128,173,223$, and $300 \mathrm{~K}$ on quenched samples at constant strain rate, $7 \times 10^{-4} \mathrm{sec}^{-1}$. The following dynamic effects due to increasing nitrogen in lattice solution were found: i) the temperature dependence of the flow stress at strains beyond the end of the Luder's plateau decreased, ii) serrated flow occurred at $300 \mathrm{~K}$, but not at lower temperatures, iii) elongation to maximum load decreased at $300 \mathrm{~K}$, but increased at $173 \mathrm{~K}$, and iv) the magnitude of the yield drop at $173 \mathrm{~K}$ decreased substantially. These dynamic effects are interpreted in terms of a change in the relative mobility of nitrogen atoms and dislocations between 128 and $300 \mathrm{~K}$.

Corliss, E. L. R., Hearing aids, Nat.Bur. Stand. (U.S.) Monogr. 117, 26 pages (Oct. 1970) 35 cents, SD Catalog No. C13.44:117.

Key words: Audition; communications; hearing; hearing aids; selection of hearing aids; speech communication.

This publication contains information, useful to the hard of hearing, on several topics relating to hearing and hearing aids. It is assumed that the individual has already consulted a physician on the diagnosis of his hearing loss, for this is the necessary first step in correcting any faulty hearing condition. The publication may also be of interest to teachers and others wishing to explore this field.

This publication represents an extensive revision of NBS Circular 534, Hearing Aids. It includes new material based upon research conducted at the National Bureau of Standards during the intervening years.

Davis, M. M., Bronsted acid-base behavior in "inert" organic solvents, Chapter 1 in The Chemistry of Nonaqueous Solvents, III, 1-135 (Academic Press Inc., New York, N. Y., 1970).

Key words: Acid-base behavior; acidity and basicity scales; aprotic organic solvents; hydrogen bonding; titrations.
A unified picture of acid-base behavior in aprotic organic solvents is presented, based on an extensive survey of the literature and experimental results of the author and associates. Evidence given to support this picture includes data pertaining to colligative properties of acids, bases, and salts and also conductance, dielectric constants, distribution between immiscible solvents, and spectral absorption in the infrared, visible, and ultraviolet. The acids upon which attention is centered are proton-donor compounds that are measurably ionized in water, such as aliphatic and aromatic carboxylic acids, substituted phenols, and mineral acids. The bases of principal interest are likewise compounds capable of forming ions in water, for example, aliphatic and aromatic amines and derivatives of guanidine or pyridine. The solvents emphasized are hydrocarbons and halohydrocarbons, but data for dipolar aprotic solvents (for example, acetone, acetonitrile, and nitrobenzene) are included. Contrasts in acid-base behavior and in acidity and basicity scales in aprotic and water-like solvents are discussed.

The role of hydrogen bonding in aprotic solvents is discussed at length. Important types of hydrogen-bonded structures include chelate rings; self-associated acids, bases, and salts; hydrogenbonded ion pairs; and homo- and heteroconjugate cations and anions. Examples are given in which hydrogen bonding of these types affects such properties as the absorption spectrum of a salt, the catalytic effect of an acid, and the accurate location of a titration endpoint.

Dehl, R. E., Collagen: Mobile water content of frozen fibers, Science 1 70, 738-739 (November 13, 1970).

Key words: Adsorbed water; calorimetry; collagen; frozen collagen; mobile water; NMR.

The NMR spectra of frozen wet collagen fibers indicate the presence of a considerable amount of unfrozen, mobile water. The quantity of mobile water may be estimated from the approximate convergence of the low temperature NMR splitting constants. An independent estimate of the mobile water content by calorimetry agrees well with the NMR estimate.

Dick, C. E., Lucas, A. C., K-shell fluorescence yields for light elements, Phys. Rev. A, 2, No. 3, 580-586 (September 1970).

Key words: Beryllium; boron; carbon; fluorescence yield; fluorine; K-shell; magnesium; $\omega_{K}$

The K-shell fluorescence yield, $\omega_{\mathrm{K}}$, has been measured for the low atomic number elements beryllium, boron, carbon, fluorine and magnesium. The primary vacancies in the $\mathrm{K}$-shell were produced by an intense beam of $\mathrm{K}$ x-rays generated by electron bombardment of aluminum and carbon targets. The measured values of $\omega_{\mathrm{K}}$ agree quite well with values calculated from a theoretical prediction of Wenzel. They exhibit only fair agreement with semi-empirical formulas which include screening and relativistic effects and with a recent calculation by McGuire based on the K-shell Auger transition rate.

Dobbyn, R. C., Williams, M. L., Cuthill, J. R., McAlister, A. J., Occupied band structure of $\mathrm{Cu}$ : Soft-x-ray spectrum and comparison with other deep-band-probe studies, Phys. Rev.B. 2, No.6, 1563-1575 (September 15, 1970).

Key words: Band structure; Cu; deep probes; emission; occupied bands; soft x-ray.

We report a new measurement of the soft $\mathrm{x}$-ray $\mathbf{M}_{2,3}$ emission spectrum of $\mathrm{Cu}$, using improved experimental techniques. Previously unreported fine structure was observed in the spectrum. Although exact correction for satellite and subband overlap and self-absorption effects is not yet possible, careful consideration has been given them, with the result that the $\mathrm{M}_{3}$ band profile can be resolved from the accompanying structure in a plausible way. Its features can be taken with reasonable confidence to be characteristic of the true $\mathrm{M}_{3}$ profile. Comparison is made with the complementary $\mathrm{L}_{3}$ soft $\mathrm{x}$-ray profile, with band theoretical estimates of both experimental x-ray profiles, and with the results of ultraviolet photoemission, x-ray photoemission and ion neutralization measurements. These comparisons favor a single particle description of the occupied bands of $\mathrm{Cu}$. 
Douglas, T. B., New approach for evaluating lattice-configurational thermodynamic properties, J. Chem. Phys. 51, No. 7 , 2831-2839 (October 1, 1969).

Key words: Configurational thermodynamic properties; excluded volume; extrapolations; lattice models; non-combinatorial method; statistical equilibrium.

As a generalization of earlier work of the author [J. Chem. Phys. 40, 2248 (1964); 45, 1080 (1966)] a completely noncombinatorial method is derived which gives for any state the exact thermodynamic properties of a system of particles on a lattice infinite in one dimension. The method appears to be essentially equivalent to, but somewhat simpler in development and application than, the matrix method used for the same problems recently. Interaction of unit configurations is explicitly considered only at lattice boundaries, leading directly to a set of independent algebraic equations giving the complete solution for a special state. Two simple examples are given to which alternative combinatorial procedures can be readily applied without approximations, yielding identical explicit results. The general method first given is modified for the imposition of artificial density constraints, and as an illustration it is shown how this variation improves markedly, over the range of disordered densities, the convergence to an infitine plane of the hard-core square-lattice fluid with nearest-neighbor exclusion.

Durst, R. A., Pick an ion, any ion, Ind. Res. pp 36-39 (November 1970). Evenson, K. M., Wells, J. S., Radford, H. E., Infrared resonance of $\mathrm{OH}$ with the $\mathrm{H}_{2} \mathrm{O}$ laser: $A$ galactic maser pump? Phys. Rev. Letters 25, No. 4, 199-202 (July 27, 1970).

Key words: Electrode monitoring; industrial monitoring; ion-selective electrodes; pollutant monitoring.

A brief discussion is given of the types of ion-selective electrodes and the basic principles of their operation. The advantages of these sensors for industrial and environmental pollution monitoring are given, such as, the continuous direct-reading nature of the measurement, fast response time, and portability. Examples of typical industrial and environmental monitoring applications are included, for example, sulfide ion in pulp processing, nitrogen oxides and fluoride in air pollution, and nitrate in soil slurries. Care is necessary in applying these sensors to complex systems where interferences could be a problem and ingenuity is required in modifying the analysis parameters to obtain reliable results. The specificity and sensitivity of these sensors to several serious pollutants will probably result in their future use in pollution-monitoring systems.

Egelstaff, P. A., Cooperative rotation of spherical molecules, J. Chem. Phys. 53, No. 7, 2590-2598 (October 1970).

Key words: Diffusion; light scattering; neutron scattering; plastic crystal; rotational; spherical molecules.

The rotational diffusion of molecules in the liquid and solid states can be studied by radiation scattering experiments. Both neutron and light scattering data are interpreted, conventionally, on the basis of rotation of single molecules, although in dense systems co-operative rotation is a possibility. It is pointed out that a comparision of incoherent neutron data and depolarised light data allows the cooperative nature of molecular rotational diffusion to be verified. The method is applied to the plastic crystal phase of cyclohexane, and it is shown that individual molecules rotate about 10 times as rapidly as the relaxation rate of the mean orientation of a group of molecules.

Florin, R. E., Wall, L. A., Small and large radicals in thin-film polymer depolymerization, Macromolecules 3, No. 5, 560-566 (September-October 1970).

Key words: Diffusion effects free radicals; polymer decomposition; polymer depolymerization; polymer irradiation; polymer pyrolysis.

Rates of polymer decomposition are enhanced or retarded as a result of the escape of small free radical or other species through the surface of thin films. Solutions for the rate equations are given as a function of thickness for a mechanism involving the competitive diffusion of small and large radicals. The enhancement of the rates of depolymerization of polytetrafluoroethylene observed with thin films is explained by the model. Comparison of the experimental results with theory indicates that the irradiation of polytetrafluoroethylene produces comparable amounts of both large and small radicals. The mechanisms discussed are undoubtedly operative in many other situations involving the radiolysis or pyrolysis of polymers, for example the radiation induced crosslinking of polymers.

Franklin, A. D., Marzullo, S., Orientation kinetics of $\mathbf{G} \mathbf{d}^{3+}-\overline{\mathbf{F}}^{-}$ interstitial pairs in $\mathbf{C a F}_{2}, J$. Phys. C. Solid State Phys. Letters to Editor 3, L171-L174 (September 1970).

Key words: Calcium fluoride; defect dipoles; dielectric relaxation; ESR line broadening; gadolinium; orientational relaxation.

The ESR spectrum from substitutional $\mathrm{Gd}^{3+}$ ions with adjacent trapped $\mathrm{F}^{-}$interstitials broadens significantly above about $100{ }^{\circ} \mathrm{C}$. The temperature-dependent part of the relaxation time associated with this broadening is compatible with the relaxation time derived by dielectric relaxation measurements on identically-treated crystals. These results are interpreted in terms of the orientational diffusion of the trapped $F^{-}$interstitial ion around the immobile $\mathrm{Gd}^{3+}$ ion on a cation site. The relaxation time is given by $\tau=\tau_{0} \exp (Q / k T)$, with $\tau_{0}=8.2 \times 10^{-14} \mathrm{~s}$ and $Q=0.38 \mathrm{eV}$.

Freeman, D. H., Goldstein, S., Schmuckler, G., Homogeneous sulfonation of styrene-divinylbenzene copolymers with oleum in organic solvents, Istael J. Chem. 7, No. 6, 741-749 (1969).

Key words: Cation exchangers; ion exchangers; oleum; sulfonation.

In order to obtain homogeneously sulfonated cation exchangers, the copolymers were sulfonated by oleum in a mixture of methylenechloride and nitro-methane. The influences of the chemical interaction and of the diffusion process on the kinetic behavior of these systems were investigated, and it was shown that raising the temperature of the sulfonation mixture markedly increases the chemical reaction rate, while the effect on the diffusion is small. A curve-fitting technique was used for the interpretation of the degree-of-sulfonation-vs.-time curves. These investigations served as guidelines for the subsequent preparation of homogeneous highly sulfonated copolymers.

French, B. M., Walter, L. S., Heinrich, K. F. J., Quantitative mineralogy of an Apollo 11 lunar sample (Proc. Apollo 11 Lunar Science Conf., Houston, Texas, January 1970), Geochim. Cosmochim. 34, Suppl. 1, 433-444 (February 1970).

Key words: Apollo 11; electron probe analysis; mineralogy; moon; petrography; rocks.

Petrography and mineral analyses of Sample 10017, collected by the Apollo 11 mission, are reported. They indicate formation by relatively rapid crystallization of a silicate melt. Major components are clinopyroxene, plagioclase and ilmenite. Minor components include troilite, native iron, and apatite. The mineral compositions indicate differentiation during magmatic crystallization, leading to Fe enrichment in the pyroxenes and to alkali enrichment in the feldspars. The mesostasis, the residual liquid produced during crystallization, is strongly enriched in $\mathrm{K}$ and $\mathrm{Si}$, but strikingly low in $\mathrm{Na}$; this may be due to volatilization during the later stages of crystallization. The compositional trends, similar to those in other lunar samples, indicate formation by relatively rapid magmatic crystallization, which developed significant chemical fractionation, at low oxygen fugacity and under virtually anhydrous conditions.

Gebbie, H. A., Kuhn, P., Bohlander, R. A., Scattering by high cirrus: Its effect on submillimeter wave determinations of atmospheric waver vapor, Nature, Letter to Editor 226, No. 5242, 71-72 (April 4, 1970).

Key words: Fourier spectroscopy; high cirrus; submillimeter waves; water vapor.

Submillimeter waves and, by implication in this context, the use of the pure rotation band, offer important advantages for determining the distribution of water vapor with height in the atmosphere. The 
reasons are these. 1) We can readily resolve single rotation lines in this region by Fourier Spectroscopy using interferometers. The calibration for intensity of single lines is easily made and a range of lines with different absorption strengths can then be chosen to match the requirements of the inversion procedure used to construct the model radiating atmosphere. 2) The use of long wavelengths allows us to dispense with hot radiation sources and use, for example, the radiation exchange between a room temperature detector and cold sky.

Gills, T. E., Marlow, W. F., Thompson, B. A., Determination of trace elements in glass by activation analysis using hydrated antimony pentoxide for sodium removal, Anal. Chem. 42, 1831-1833 (December 1970).

Key words: Activation analysis; glass; hydrated antimony pentoxide (HAP); radiochemical separations; sodium; trace elements.

A method has been developed for the determination of a number of trace elements at the ppm and ppb levels in high-sodium glass by neutron activation analysis. For glass containing about $10 \%$ sodium, neutron irradiation at levels sufficient to allow determination of trace constituents results in production of $10 \mathrm{mCi}$ or more of ${ }^{24} \mathrm{Na}$. If the ${ }^{24} \mathrm{Na}$ is allowed to decay before analysis, information about elements producing short-lived radioisotopes is lost. In the procedure described the radiosodium is removed by passing the dissolved glass through a column of hydrated antimony pentoxide (HAP). The effluent can be counted directly with a $\mathrm{Ge}(\mathrm{Li})$ or $\mathrm{NaI}(\mathrm{Tl})$ detector. The method has been applied to the analysis of NBS Standard Reference Material (SRM) glass which was found to contain $1 \mathrm{ppm}$ of $\mathrm{Cu}$ and sub-ppm levels of $\mathrm{Mn}, \mathrm{Au}, \mathrm{Ga}, \mathrm{Sb}, \mathrm{La}, \mathrm{Co}$, and Ir.

Glass, A. J., Guenther, A. H., Editors, Damage in laser materials, Proceedings of a Symposium Sponsored by the American Society for Testing and Materials and by the National Bureau of Standards June 24-25, 1970, NBS, Boulder, Colorado, Nat. Bur. Stand. (U.S.), Spec. Publ. 341, 133 pages (Dec. 1970) \$1.25, SD Catalog No. C13.10:341)

Key words: Ferroelectric materials; glass; laser damage; lasers.

The second ASTM Symposium on Damage in Laser Materials was held at the National Bureau of Standards at Boulder, Colorado on June 24th and 25th, 1970. About 150 attendees heard papers by fourteen speakers, representing the major manufacturers of laser glass, government, industrial and university laboratories. Although the primary emphasis was on $\mathrm{Nd}$ :glass as a laser material, damage mechanisms and morphology in ruby, sapphire, and nonlinear optic materials were also considered. It was clear that substantial advances have been made in the last year in the production of damage-resistant laser glass for use in conventional $Q$-switched lasers. New diagnostic techniques have been developed, and in some cases, adopted as quality control procedures by the glass manufacturers. Theoretical understanding of the fundamental damage mechanisms is beginning to emerge, and material properties relevant to these damage mechanisms are beginning to be measured. It was equally clear that a great deal of effort remains to be expended to develop techniques suitable for nondestructive testing of laser materials, and that many properties of laser materials at high energy density are presently poorly understood.

Glaze, D. J., Improvements in atomic cesium beam frequency standards at the National Bureau of Standards, IEEE Trans. Instr. Meas. IM-19, No. 3, 156-160 (August 1970).

Key words: Atomic frequency standard; cesium beam; figure of merit; frequency accuracy; frequency multiplier; frequency precision; NBS-III; NBS-5; phase noise; quartz crystal oscillator; ramsey cavity; slave oscillator.

The National Bureau of Standards Frequency Standard, NBS-III, a cesium beam with a 3.66 meter interaction region, has been in operation since 1963. The last published (1966) accuracy capability for NBS-III was $1.1 \times 10^{-12}(1 \sigma)$. With this performance NBS-III was used in the measurment of the frequency of the hyperfine separation of hydrogen. This was, and is, the most accurate published measurement of any physical quantity. A number of improvements are being made in NBS-III to improve its accuracy capability. In early 1966 the vacuum system was modified to use three commercial 200 liter per second ion pumps. The resulting ultimate pressure was improved by a factor of ten to about $10^{-8}$ torr $\left(1.3 \times 10^{-6}\right.$ $\mathrm{N} / \mathrm{M}^{2}$ ). Several new solid-state, broadband frequency multiplier chains have been constructed with particular attention given to reduction of both power-line related sideband levels and random phase noise sideband levels. Reduction of the random phase noise by more than $20 \mathrm{~dB}$ compared to the previous state of the art has been obtained consistently. One of these frequency multiplier chains is presently in use in the 5 to $60 \mathrm{MHz}$ section of the $9.192 \ldots \mathrm{GHz}$ excitation system. In addition a solid-state servo system has been employed to control the frequency of the $5 \mathrm{MHz}$ slave oscillator. This servo system and the new frequency multiplier chain have improved both the reliability and long-term stability of NBS-III.

Comparisons were made between NBS-III and one of the commercial cesium standards in the NBS Clock Ensemble. The relative fractional frequency stability of $\sigma(N=2, \tau=1$ day, $T=7$ days $)$ $=1 \times 10^{-13}$ was observed for nine weekly comparisons. Presently at NBS there are no hydrogen masers or thallium beams capable of being used in meaningful measurements against NBS-III. The very long-term frequency stability for this recently improved NBSIII system has not been evaluated fully. Due to the improvements in both electronics systems and evaluative techniques, however, an accuracy of $5 \times 10^{-13}(1 \sigma)$ for a single evaluative experiment is reported. Substantial NBS effort is being expended toward improvement of the accuracy and figure of merit (presently 10) for NBS-III. New designs and associated components for improved beam optics have been completed. Improved, completely solid-state $5 \mathrm{MHz}$ to $9.2 \mathrm{GHz}$ frequency multipliers have been constructed, and testing is being conducted now. The modified NBS-III beam tube together with new electronic systems will be designated NBS-5. It is expected to be in operation in early 1970 and to exhibit a figure of merit in excess of 500 .

Groth, H. G., Wellmann, P., Editors, Spectrum formation in stars with steady-state extended atmospheres, Proceedings of the International Astronomical Union Colloquium No. 2, Commission 36, Munich, Germany, April 16-19, 1969, Nat. Bur. Stand. (U.S.), Spec. Publ. 332, 342 pages (August 1970) \$1.75, SD Catalog No. C13.10:332.

Key words: Extended atmospheres; interpretation of stellar spectra; line formation.

In section 1 practical details concerning the equivalence of observational and theoretical descriptions of stellar spectra are reviewed, particularly the difficulty of identifying the observed reference level (continuum) with the theoretical continuum in the case when many lines are present. In this connection thought must be given to how integrals over frequency should be normalised and evaluated because the effective continuous absorption coefficient does not remain constant over the range from 0 to $\infty$. The choice of spectroscopic details by which to determine $\mathrm{T}_{\text {eff }}, \log g$ and abundances requires careful consideration.

In section 2 the factors by which an extended atmosphere are recognized are summarized and the question is posed do all stars have extended atmospheres. Another question requiring an answer is whether the concepts microturbulence and macroturbulence are physically real concepts or whether they are merely fitting parameters to make a simple LTE theory account for the observed spectra of supergiants in which rather wide lines occur and many multiplets show rather steep gradients. In section 3 the types of line sensitive to non-LTE conditions are described. These are resonance lines, lines arising from metastable levels, subordinate lines for which the upper level is sufficiently separated from the continuum and other levels that this upper level is chiefly populated by radiative processes from the ground or other low lying levels and lines which go into emission in low density atmospheres as a result of optical-pumping (fluorescent) processes. Such lines should not be used for abundance determinations by means of LTE theory though this is frequently done.

Theoretical considerations are discussed in section 4 where first the problem of the two-level atom is sketched and then the problem is generalised to a many-level atom. The parameter $\lambda$ which gives the probability that a photon is lost from the line by de-excitation processes other than spontaneous emission is defined and it is pointed out that non-LTE physics has the effect of adding a scattering 
term to the expression for the source function. One example is given of the effect of changing the line source function from the Planck function to a form suitable for isotropic coherent scattering. The line becomes deeper and wider for the same number of atoms. Interpretational problems in stellar spectra are discussed in section 5 . It is noted that many lines in main-sequence early type spectra show the effects of departures from LTE. These effects are shown to a conspicuous degree by the spectra of shell stars. The example of He I 5876 in 10 Lacertae, $09 \mathrm{~V}$, is discussed and the implication for interpreting the $\mathrm{He} \mathrm{I}$ lines in all B type main-sequence stars are touched upon. Helium-weak and helium-strong spectra probably indicate variations in density of the outer atmosphere rather than true abundance differences. The spectra of supergiants are also considered and it is pointed out that the Ia supergiants of type B may be hydrogen-poor.

Finally in section 6 the problem of choosing simplified physical representations of line forming when non-LTE physics must be used is discussed. Some relevant points concerning the observed spectral lines used for spectral classification are illustrated by means of partial energy-level diagrams.

Guttman, C. M., DiMarzio, E. A., Separation by flow. II. Application to gel permeation chromatography, Marcromolecules 3, No. 5, 681-691 (September-October 1970).

Key words: Chromatography; gel filtration; gel permeation chromatography; macromolecules; separation by flow.

Models of a Gel Permeation Chromatography column are proposed in which there is flow through each of the beads as well as around them. Diffusion is allowed within and outside of the beads. By making general arguments on particle current flow, the volume elution is computed as a function of solute particle size for a simplified view of the column. The equation for the location of volume elution peaks thus derived show functional dependences on the particle radius and the column geometry very much like equations derived by previous workers for models in which there was no flow in the beads. The method of Herman's is extended to allow for flow within the beads. Two times characterize the system; the time for a particle to diffuse into and out of the bead and the time to flush the particle out of the bead. The width of the volume elution peak (in contradistinction to the work of Herman's) no longer becomes infinite as the diffusion coefficient goes to zero since the residence time within the bead is never larger than the flush time. Explicit formulae are given for the first three moments of the volume elution; it is shown that the elution volume for a monodispersed species is gaussian. In all cases systems with open pores which allow flow show better separation capabilities than those which do not allow flow.

Haar, L., Shenker, S. H., A phenomenological equation of state, Proc. 5th Symp. Thermophysical Properties, Boston, Mass., September 30-October 2, 1970, C. F. Bonilla, Ed., pp. 223-226 (American Society of Mechanical Engineers, New York, N.Y., 1970).

Key words: Compressibility factors; equation of state; high density; high temperature; pairwise potentials; Redlich Kwong; second virial coefficient; thermodynamics.

A simple phenomenological equation of state for high temperature gases is proposed. It is developed from considerations of the two body pair interactions, and it has no arbitrary parameters. The equation has been applied to two simple gases, nitrogen and argon. The results compare quite well with experimental data up to 10,000 bar and in the range of two to four times the critical temperature. Also included for comparison is the widely used Redlich Kwong equation. The present work compares favorably with the Redlich Kwong at the lowest-temperature; however, as temperature is increased the present work becomes increasingly more accurate, whereas the corresponding states equation tends to degrade with temperature.

Hastie, J. W., Hauge, R. H., Margrave, J. L., High temperature chemistry: Stabilities and structures of high temperature species, Ann. Rev. Phys. Chem. 21, 475-498 (1970).

Key words: High temperature species; stability structure.

Recent experimental and theoretical work on the stabilities and structures of high temperature species has been reviewed. Emphasis was given to data which may be used for the thermodynamic characterization of a high temperature species, such as molecular geometry vibrational and electronic energy levels. The techniques from which stability and structural data have been obtained for high temperature species are also discussed. These include, Knudsen effusion and photoionization mass spectrometry, matrix isolation infrared spectroscopy, electron diffraction, electric dipole deflection, electronic spectroscopy, microwave spectroscopy and semiempirical and abinitio molecular orbital calculations.

Hayward, E., Photonuclear reactions, Nat. Bur. Stand. (U.S.), Monogr. 118, 46 pages (August 1970) 50 cents, SD Catalog No. C13.44:118.

Key words: Nuclear hydrodynamics; particle-hole calculations; photon scattering; photonuclear; sum rules.

This paper reviews photonuclear reactions in the approximate energy range 10 to $30 \mathrm{MeV}$. Various sum rules are discussed and applied to experimental data. Several different theories are described and their predictions compared with experiment, and as often as possible open questions and discrepancies are pointed out.

Hirshfeld, A. T., Hoppes, D. D., Transition mixing ratios determined from a study of the electron and gamma-ray distributions from oriented ${ }^{192} \mathbf{I r}$, Phys. Rev. C 2, No. 6, 2341-2349 (December 1970).

Key words: Beta rays, angular distribution; gamma rays; hyperfine field in iron; ${ }^{192} \mathrm{Ir}$, magnetic moment; nuclear orientation; transition missing ratios.

The angular distributions measured for 12 gamma rays in ${ }^{192} \mathrm{Os}$ and ${ }^{192} \mathrm{Pt}$ resulting from the decay of cryogenically oriented ${ }^{192} \mathrm{Ir}$ have been used to determine the following E2/M1 mixing ratios: $201 \mathrm{keV}$, $\delta^{2}>3.7 ; 296 \mathrm{keV}, \delta=-\left(6_{-1}^{+3}\right) ; 308 \mathrm{keV}, \delta=-(7.1 \pm 0.6) ; 417 \mathrm{keV}$, $\delta=4_{-3}^{+7} ; 485 \mathrm{keV}, \delta=5.8 \pm 0.8 ; 604 \mathrm{keV}, \delta=1.5 \pm 0.1$, following the Rose-Brink sign convention. The angular distribution of the $672 \mathrm{keV}$ beta group and the above gamma measurements limit the relative contribution, $I_{L}$, of different operator tensor ranks, $L$, involved in each beta transition. The results for electron capture transitions terminating at levels in osmium are: $694 \mathrm{keV}$ level, $I_{1}>0.9 ; 584 \mathrm{keV}$ level $I_{0}>0.6, I_{2}<0.1$. For beta transitions feeding platinum states, we determine: $921 \mathrm{keV}$ level, $I_{1}>0.8 ; 785 \mathrm{keV}$ level, $I_{1}>0.92$, $I_{2}<0.03$. The ${ }^{192} \mathrm{Ir}$ ground state magnetic moment is positive.

Jacox, M. E., Milligan, D. E., Matrix-isolation study of the vacuum-ultraviolet photolysis of methyl chloride and methylene chloride. Infrared and ultraviolet spectra of the free radicals CCI, $\mathbf{H}_{2} \mathbf{C C I}$, and $\mathbf{C C I}_{2}, J$. Chem. Phys. 53, 7, 2688-2701 (October 1, 1970).

Key words: $\mathrm{CCl}$; $\mathrm{CCl}_{2}$; force constants; free radical; $\mathrm{HCCl} ; \mathrm{HCCl}_{2}$; $\mathrm{H}_{2} \mathrm{CCl}$; infrared spectrum; matrix isolation; photolysis; ultraviolet spectrum.

Infrared and ultraviolet spectroscopic studies have been conducted on the products of the vacuum-ultraviolet photolysis of normal and isotopically substituted methyl chloride and methylene chloride isolated in argon and nitrogen matrices at $14 \mathrm{~K}$. The cage effect has been formed to inhibit halogen detachment processes in these sys. tems. Infrared absorptions attributed to $\mathrm{CCl}, \mathrm{HCCl}$, and $\mathrm{H}_{2} \mathrm{CCl}$ and the 2800-A ultraviolet absorption of $\mathrm{CCl}$ appear in the methyl chloride photolysis studies. Observation of a "negative anharmonicity" for the lowest frequency infrared absorption of $\mathrm{H}_{2} \mathrm{CCl}$ can be explained by postulating that the molecule is planar. The C-Cl stretching force constant of $\mathrm{H}_{2} \mathrm{CCl}$ is exceptionally high. The implications of $(p-d) \pi$ bending, which may account for the large C-Cl stretching force constant, are explored. An absorption near $2300 \AA$ behaves appropriately for assignment to a second electronic transition of $\mathrm{CCl}$, providing support for the previous tentative assignment of a group of emission bands to such a transition. Photolysis of methylene chloride in a matrix environment leads principally to the stabilization of $\mathrm{CCl}_{2}$. A revised estimate of the force constants of $\mathrm{CCl}_{2}$ is given.

Johnson, D. R., Powell, F. X., Microwave detection of thioformaldehyde, Science 169, 679-680 (August 14, 1970). 
Key words: Astronomically important; electrical discharge; interstellar medium; microwave spectroscopy; rotational spectrum; thioformaldehyde.

Thioformaldehyde $\left(\mathrm{H}_{2} \mathrm{CS}\right)$ has been detected and characterized from its microwave spectrum. Preliminary analysis of rotational transitions for $\mathrm{H}_{2} \mathrm{C}^{32} \mathrm{~S}$ shows this new species to have $\mathrm{C}_{2 v}$ symmetry with rotational constants of $A=292729 \mathrm{MHz}, B=17698 \mathrm{MHz}$, and $C=16652$ $\mathrm{MHz}$. The possibility of detection of thioformaldehyde in the interstellar medium is discussed and a table of transitions expected to be of importance there is presented.

Krauss, M., Potential energy surfaces, Ann. Rev. Phys. Chem. 21,39-46(1970).

Key words: Activation energy; configuration interaction; correlation energy surface; Hartree-Fock; $\mathrm{H}_{3}^{+} ; \mathrm{H}_{3} ; \mathrm{H}_{3}^{-} ; \mathrm{H}_{4} ; \mathrm{LiH}_{2}$; $\mathrm{LiHF} ; \mathrm{HNO}$; $\mathrm{HeH}_{2}^{+}$; proton transfer.

Ab initio energy surfaces applicable to chemical kinetics and collisional phenomena are reviewed. The calculations are divided into two categories, Hartree-Fock and beyond. It is noted that HartreeFock calculations are routine but that correlation techniques are necessary for reaction collisions. Definitive work exists for $\mathrm{H}_{3}^{+}$ and $\mathrm{H}_{4}$ but the activation energy of $\mathrm{H}_{3}$ is still inaccurate relative to the scattering calculational requirements. Other qualitative work is described for $\mathrm{H}$ or $\mathrm{Li}$ interaction with diatomics.

Lafferty, W. J., Microwave spectrum, dipole moment, and conformation of cyclopentene oxide, J. Mol. Spectry. 36, No. 1, 84-93 (October 1, 1970).

Key words: Cyclopentene oxide; dipole moment; epoxy cyclopentene; microwave spectrum; ring conformation; rotational constants.

The microwave spectrum of cyclopentene oxide has been studied. Rotational constants for the ground state and two excited vibrational states of one ring conformation have been obtained. Ground state constants are $A_{0}=5709.38 \pm 0.02, B_{0}=4541.12 \pm 0.02$ and $C_{0}=3248.97 \pm 0.02$ (errors are 2 standard deviations). The dipole moment components of the molecule are $\mu_{a}=1.16_{3} \pm 0.033$, $\mu_{c}=1.63_{0} \pm 0.031$, and $\mu=2.00_{2} \pm 0.015$. The rotational constants and dipole moment components obtained experimentally can be satisfactorily explained only if the boat form is the most stable conformation of the ring.

Lamb, V. A., Evidence for a complex chloromolybdate ion in a molten salt medium from transference experiments, J. Electrochem. Soc. 117, No. 10,1269-1270 (October 1970).

Key words: Hexachloromolybdate complex ion; molten salt; transference numbers.

Transference experiments have been performed with solutions of potassium hexachloromolybdate in molten eutectic mixtures of potassium chloride and lithium chloride. The results indicate that a complex molybdenum-containing anion exists at $800^{\circ} \mathrm{C}$.

Latanision, R. M., Westwood, A. R. C., Surface- and environment-sensitive mechanical behavior, Chapter in Advances in Corrosion Sciences and Technology, 1, 51-145 (Plenum Press, New York, N.Y., 1970).

Key words: Complex-ion embrittlement; dislocations; liquid metal embrittlement; surface; surface active agent.

The influences of surface structure and environment on the mechanical behavior of crystalline inorganic solids are reviewed and possible mechanisms discussed. In particular, the various roles of such factors as the atomic, electronic, and defect structure of the nearsurface regions, the presence of adsorbed surface-active species, alloyed layers, oxide films, gaseous or liquid environments, etc. are considered in connection with the Roscoe, Rebinder, and Joffe effects, liquid-metal embrittlement, complex-ion embrittlement, hydrogen embrittlement, and other phenomena.
Ledbetter, H. M., On the martensite erystallography of the cubic to orthorhombic transformation in Au-47.5 Cd, Scripta Met. 4, No. 11,931-937 (November 1970).

Key words: Crystallography; gold-cadmium alloy; martensite; phase transformations; twinning.

Complete calculations of the geometrical features of the cubic to orthorhombic martensitic transformation in the Au-47.5 Cd alloy system have been made for both Type I and Type II transformation twinning. None of the usual crystallographic features are sufficiently sensitive to distinguish between these two possible modes of lattice invariant deformation. Alternatives are offered which are believed to be experimentally simpler than those previously proposed.

Lesclaux, R., Searles, S., Sieck, L. W., Ausloos, P., Irradiation of cyclohexene with 8.4, 10.0 and $11.6-11.8 \mathrm{eV}$ photons. Dissociation of neutral excited cyclohexene and reactions of $\mathbf{C}_{6} \mathbf{H}_{10}^{+}$, J. Chem. Phys. 53, No. 8, 3336-43 (October 15, 1970).

Key words: Cyclohexene; ion-molecule reactions; photoionization; photolysis; unimolecular-/dissociation.

The photolysis of cyclohexene has been investigated at photon energies above $(10.0 \mathrm{eV}, 11.6-11.8 \mathrm{eV})$ and below $(8.4 \mathrm{eV})$ its ionization potential $(8.9 \mathrm{eV})$. The ionic processes were studied in a mass spectrometer equipped with a photon source. The parent $\mathrm{C}_{6} \mathrm{H}_{10}^{+}$ion was seen to undergo the following reactions with cyclohexene:

$\mathrm{C}_{6} \mathrm{H}_{10}^{+}+$cyclo $-\mathrm{C}_{6} \mathrm{H}_{10} \rightarrow$ cyclo $-\mathrm{C}_{6} \mathrm{H}_{12}+\mathrm{C}_{6} \mathrm{H}_{8}^{+} k=0.7$

$\times 10^{-10} \mathrm{~cm}^{3} /$ molecule-sec $\rightarrow \mathrm{C}_{12} \mathrm{H}_{20}^{+} k=4.1 \times 10^{-10} \mathrm{~cm}^{3} /$ molecule-sec

Analysis of the yields of cyclohexane formed in the first of these reactions in the photolysis at $10.0 \mathrm{eV}$ over the pressure range from 1 to 10 torr indicates that the relative importance of the two reactions is the same in this pressure range as in the ion source of the mass spectrometer at a pressure of $10^{-2}$ torr. At $11.6-11.8 \mathrm{eV}$, the parent ion also dissociates, mainly to form $\mathrm{C}_{5} \mathrm{H}_{7}^{+}$and $\mathrm{CH}_{3}$. Evidence is presented which indicates that at these energies, the majority of the $\mathrm{C}_{6} \mathrm{H}_{10}^{+}$ions retain a cyclic structure. The neutral, electronically excited cyclohexene molecule dissociates mainly as follows:

$\mathrm{C}_{6} \mathrm{H}_{10}^{*} \rightarrow 1,3-\mathrm{C}_{4} \mathrm{H}_{6}+\mathrm{C}_{2} \mathrm{H}_{4}$, at $8.4 \mathrm{eV}, \Phi=0.82$

$$
\rightarrow \mathrm{C}_{5} \mathrm{H}_{7}+\mathrm{CH}_{3} \text {, at } 8.4 \mathrm{eV}, \Phi=0.08 \text {. }
$$

The photolysis of $3,3,6,6-d_{4}$-cyclohexene demonstrated that the first of these processes occurs predominantly by the "symmetrical" cleavage of the $3-4$ and $5-6 \mathrm{C}-\mathrm{C}$ bonds, at all energies.

Levin, E. M., Liquid immiscibility in oxide systems, Chapter in Phase Diagrams Materials Science and Technology, 3, The Use of Phase Diagrams in Ceramics, Glass and Metal Technology, pp. 143-236 (Academic Press, Inc., New York, N.Y., 1970).

Key words: Glass chromatography; immiscibility and courses of crystallization; immiscibility and practical applications; immiscibility and thermodynamics; immiscibility in oxides; liquid immiscibility; $\mathrm{Ln}_{2} \mathrm{O}_{3}-\mathrm{B}_{2} \mathrm{O}_{3}$ phase diagrams; mechanism of phase separation; metastable liquid immiscibility; microphase separation; phase diagrams of metastable immiscibility; spinodal; structural interpretation of immiscibility; super-duty silica brick.

Stable and metastable liquid immiscibility in oxide systems is dis cussed from the standpoint of thermodynamics, interpretation, and application. The classic Gibbs free energy of formation-composition diagrams are used to illustrate the difference between a stable and a metastable immiscibility gap and to define the spinodal. Phase equilibrium principles are applied to liquid immiscibility and typical courses of crystallization are described. Structural interpretation is applied to the occurrence and extent of immiscibility including the temperature of the monotectic and the effect of homogenizers. The general incompatibility of compound formation and liquid immiscibility is demonstrated for the rare earth oxide-boric oxide systems. Occurrence and methods of study of micro-phase separation are elucidated. Three mechanisms of microphase separation are 
discussed briefly: nucleation and growth, spinodal decomposition, and intersecting growth theory. Binary and ternary phase diagrams showing liquid immiscibility are presented. Application to the following commercial products are discussed: super-duty silica brick, Vycor glass, Pyrex glass, glass ceramics, and glass chromatography.

Levin, E. M., Roth, R. S., The system niobium pentoxidephosphorus pentoxide, J. Solid State Chem. 2, No. 2, 250-261 (1970).

Key words: $\mathrm{Nb}_{2} \mathrm{O}_{5}-\mathrm{P}_{2} \mathrm{O}_{5} ; \mathrm{NbPO}_{5} ; \mathrm{Nb}_{2} \mathrm{P}_{4} \mathrm{O}_{15} ; \mathrm{Nb}_{9} \mathrm{PO}_{25} ; \mathrm{Nb}_{44} \mathrm{P}_{2} \mathrm{O}_{115}$ $\mathrm{P}_{2} \mathrm{O}_{5}-\mathrm{Nb}_{2} \mathrm{O}_{5}$; phase diagram; $\mathrm{ReO}_{3}$-type compounds.

A partial phase diagram of the system has been determined experimentally, using the quenching technique and identifying the phases by x-ray powder diffractometry. The major phase, $\mathrm{NbPO}_{5}$, melts above $1650^{\circ} \mathrm{C}$. It transforms at about $1253^{\circ} \mathrm{C}$ from a low-temperature, tetragonal phase to a high-temperature, monoclinic phase, isostructural with $\mathrm{TaPO}_{5}$. The $9 \mathrm{Nb}_{2} \mathrm{O}_{5} \cdot \mathrm{P}_{2} \mathrm{O}_{5}$ phase shows a limited solid solution range and melts incongruently at $1435^{\circ} \mathrm{C}$. The eutectic between 9:1 and $\mathrm{NbPO}_{5}$ is at $1365^{\circ} \mathrm{C}$ and $67 \mathrm{~mol} \% \mathrm{Nb}_{2} \mathrm{O}_{5}$. At 96.4 mol \% $\mathrm{Nb}_{2} \mathrm{O}_{5}$ another phase was found, stable over only $80^{\circ} \mathrm{C}$ and isostructural with the $9: 1$ phase. A new monoclinic phase, ' $22: 1^{\circ}$ ' ss was found, with a limited stability field in both temperature and composition. In the subsystem $\mathrm{NbPO}_{5}$ one compound was identified of probable composition $\mathrm{NB}_{2} \mathrm{O}_{5} \cdot 2 \mathrm{P}_{2} \mathrm{O}_{5}$, with pseudocubic symmetry and melting incongruently at about $1025{ }^{\circ} \mathrm{C}$. X-ray powder diffraction data and unit cell dimensions are listed for the phases.

Lias, S. G., Collin, G. J., Rebbert, R. E., Ausloos, P., Photolysis of ethane at 11.6-11.8 eV, J. Chem. Phys. 52, No. 4, 1841-1851 (February 15, 1970).

Key words: Ethane; free radicals; ions; photolysis; primary processes; vacuum ultraviolet.

The photolysis of ethane, carried out with an argon resonance lamp, has been reinvestigated with the related purposes of (1) measuring quantum yields of all fragments formed in the dissociation of excited ethane, and (2) associating these fragments with the primary processes occurring in the photolysis of ethane. These, and their relative abundances are

$$
\begin{aligned}
\mathrm{C}_{2} \mathrm{H}_{6}^{*} & -\mathrm{C}_{2} \mathrm{H}_{6}^{+}, 5 \% \\
& -\mathrm{CH}_{4}+\mathrm{CH}_{2}, 16 \% \\
& -\mathrm{C}_{2} \mathrm{H}_{5}+\mathrm{H}, 41 \% \\
& -\mathrm{C}_{2} \mathrm{H}_{4}+\mathrm{H}_{2}, 23 \% \\
& -\mathrm{CH}_{3}+\mathrm{CH}_{3}, 15 \% .
\end{aligned}
$$

These results are compared with conclusions reached in earlier studies on the photolysis of ethane with xenon and krypton lamps in order to determine the effect of energy on the relative probabilities of the primary processes. It is found that direct bond scission increases in importance with increasing energy, while processes involving rearrangement decrease in importance.

The radical and molecular fragments formed in the dissociation of excited ethane were determined by (a) analyzing the products formed in $\mathrm{C}_{2} \mathrm{H}_{6}-\mathrm{C}_{2} \mathrm{D}_{6}$ - $\mathrm{NO}(1: 1: 0.1)$ mixtures and (b) $\mathrm{C}_{2} \mathrm{D}_{6}$ in the presence of $\mathrm{H}_{2} \mathrm{~S}$, which scavenges free radicals to form isotopically-unique hydrocarbon products, and (c) $\mathrm{C}_{2} \mathrm{H}_{6}$ in the presence of $\mathrm{C}_{3} \mathrm{D}_{6}$, which scavenges $\mathrm{H}$-atoms to form propyl radicals which in turn, react with radicals in the system to form characteristic products. Such experiments, as well as quantum yield determinations, were also carried out at the krypton and xenon lines.

Lias, S. G., Rebbert, R. E., Ausloos, P., Irradiation of xenonpropane and argon-propane mixtures with gamma rays and 21.2 eV photons, J. Chem. Phys. 52, No. 2, 773-783 (January 15, 1970).

Key words: Deactivation; ion-molecule reactions; photoionization; radiolysis; unimolecular ion fragmentation.

Mixtures of xenon or argon with propane $(20: 1)$ are irradiated in the presence of radical scavengers by $\gamma$-rays and by $21.2 \mathrm{eV}$ photons from a helium resonance lamp. The ion-pair yields of products originating in ionic fragmentation reactions or ion-molecule reactions are derived from isotopic analyses of product molecules formed with $\mathrm{C}_{3} \mathrm{D}_{8}-\mathrm{C}_{3} \mathrm{H}_{8}$ mixtures or with $\mathrm{CD}_{3} \mathrm{CH}_{2} \mathrm{CD}_{3}$. At a given pressure, only minor differences are seen between the radiolysis and photolysis in the modes of dissociation of parent propane ions formed by charge transfer with a particular rare gas. Higher energy ionic fragmentation processes are seen to be quenched with increasing pressure; in the case of xenon-propane mixtures, it is demonstrated that the parent ion yield increases as primary fragmentation processes are quenched. In the irradiated argon-propane-scavenger mixtures, isotope effects in the ionic fragmentation mechanism are seen which indicate that collisional deactivation of the excited deuterated fragment ions is more efficient that that of the corresponding protonated ions. In the photolytic experiments, it was deduced that the decomposition of neutral excited propane molecules constitutes about $10 \%$ of the product forming processes in the xenon mixtures, while in the radiolytic experiments, the maximum contribution of neutral excited molecule decomposition is $5 \%$. Similar deductions for the argon mixtures were not feasible.

Marshak, H., Langsford, A., Tamura, T., Wong, C. Y., Total neutron cross section of oriented ${ }_{165}$ Ho from 2 to $135 \mathrm{MeV}$, Phys. Rev. C 2, No. 5, 1862-1881 (November 1970).

Key words: Black-nucleus model; coupled channel calculation; $\mathrm{Ho}_{165}$; nuclear orientation; nuclear Ramsauer effect; nuclear shape; optical-model; total neutron cross section.

The difference in the total neutron cross section due to nuclear orientation, $\Delta \sigma_{\text {def }}$, has been measured for ${ }^{165} \mathrm{Ho}$ over the energy range of 2 to $135 \mathrm{MeV}$. The results show that $\Delta \sigma_{\text {def }}$ oscillates as well as changing sign, contrary to our classical concept of the interaction. The data, both $\sigma_{t}$ and $\Delta \sigma_{\text {def }}$, are successfully fitted by adiabaticcoupled channel calculations using the optical-model. The parameters for the optical-model were determined by fitting $\sigma_{t}$ and $\sigma_{R}$ data (over approximately the same energy range as in the present work) for the two spherical nuclei $\mathrm{Cd}$ and $\mathrm{Pb}$. The only additional quantity being introduced was the quadrupole deformation parameter for ${ }^{165} \mathrm{Ho}$ which is known to be 0.33 . The data for $\Delta \sigma_{\text {def }}$ can also be explained quite well by a simple semiempirical model which makes use of the black-nucleus model, the nuclear Ramsauer effect and the experimental $\sigma_{t}$ data. The temperature dependence of $\Delta \sigma_{\text {def }}$ agrees very well with our calculated values for the degree of nuclear orientation, which is consistent with ${ }^{165} \mathrm{Ho}$ being almost a pure quadrupole shaped nucleus.

Meshkov, S., Commentary on meson spectroscopy, Chapter in Experimental Meson Spectroscopy, C. Baltay and A. H. Rosenfeld, Ed., pp. 535-546 (Columbia Univ. Press, New York, N.Y., 1970).

Key words: $\mathrm{A}_{2}$; mesons; resonances; spectroscopy; $\mathrm{SU}(3)$; $\mathrm{SU}(6)_{\mathrm{w}}$.

A description of a method for describing the split $A_{2}$ is presented, as well as some comments on the general problem of describing mesons and analyzing their structure.

Milligan, D. E., Jacox, M. E., Infrared spectrum of the CIHClion isolated in an argon matrix, J. Chem. Phys. 53, No. 5 , 2034-2040 (September 1, 1970).

Key words: Bichloride anion; ClHCl free radical; electron attachment; HCl dimer; hydrogen bonding; infrared spectrum; matrix isolation; ultraviolet spectrum; vacuum-ultraviolet photolysis.

Upon vacuum-ultraviolet photolysis at $14 \mathrm{~K}$ of samples of $\mathrm{HCl}$ isolated in an argon matrix at mole ratios favorable for the presence of an appreciable concentration of dimeric $\mathrm{HCl}$, prominent absorptions appear at 696 and $956 \mathrm{~cm}^{-1}$. The positions, and contours, and relative intensities of these absorptions correspond within experimental error with those previously attributed to the ClHCl free radical isolated in an argon matrix. Studies of a chlorine-37 enriched sample have confirmed the presence of two chlorine atoms in the molecule. A broad, unstructured absorption at $2870 \AA$ may also be contributed by this $\mathrm{ClHCl}$ species. When a small concentration of atomic potassium or cesium is co-deposited with the Ar: HCl sample, evidence is obtained for the formation of an alkali metalcontaining complex. Photoionization of the alkali metal atoms upon exposure of the resulting sample to the radiation of a medium- 
pressure mercury are leads to the appearance of very prominent absorptions at 969 and $956 \mathrm{~cm}^{-1}$. It is proposed that these absorptions may more suitably be assigned to the $\mathrm{ClHCl}^{-}$ion, trapped in an inert, essentially non-ionic environment. Implications of this reassignment with regard to the nature of the hydrogen bonding of $\mathrm{ClHCl}^{-}$are discussed, and possible mechanisms for the production of $\mathrm{ClHCl}^{-}$under the conditions of these experiments are considered.

Moore, C. E., Ionization potentials and ionization limits derived from the analyses of optical spectra, Nat. Stand. Ref. Data Ser., Nat. Bur. Stand. (U.S.), 34, 22 pages (Sept. 1970) 75 cents, SD Catalog No. C13.48:34.

Key words: Atomic spectra, ground terms; ground terms, atomic spectra; ionization limits; ionization potentials.

A current table of ionization potentials expressed in electron volts and a detailed table giving the limits from which they have been derived are presented. For each spectrum the ground term is given, with the limit as the ground state. The energy levels of terms of the lowest configuration determined from ground state zero, are also included for selected spectra. The literature references used for each spectrum are indicated by number and listed in a bibliography with some 200 entries.

The latest recommended conversion factor $\left(\mathrm{cm}^{-1}\right.$ to $\left.\mathrm{eV}\right) 0.000123981$ corresponding to $1 \mathrm{eV}=8065.73 \mathrm{~cm}^{-1}$ has been used throughout.

Moore-Sitterly, C. E., Fundamental spectroscopic data, IAU Commission 14, August 1967 Meeting, Trans. Intern. Astron. Union 13A, 229-266 (1967). Moore-Sitterly, C. E., Rare-earth spectrathe 1966 picture, Proc. First International Conf. Spectroscopy, Bombay, India, January 9-18, 1967, 1, 1-13 (1967).

Key words: Cross section; transition probabilities; wavelength standards.

The present report summarizes important activities in the field since the Twelfth General Assembly of the Union held in Hamburg in 1964. The main topics deal with standards of wavelength, transition probabilities, line broadening, atomic collision cross sections, molecular spectra of astrophysical interest, general progress in studies of laboratory spectra and current work on the solar spectrum.

Moore-Sitterly, C. E., Silicon in the sun, Chapter in Vistas in Astronomy (Commemoration of H. N. Russell) A. Beer, Ed., 12 , 307-312 (Pergamon Press, Inc., New York, N.Y., 1970).

Key words: Atomic and ionic in the sun; silicon in the spectra of silicon; silicon spectra in the sun's atmosphere sun.

A brief survey is given of Silicon lines in the spectra of the solar photosphere, chromosphere, and corona. These lines are not only from the spectrum of the neutral atom, but arise, also, from the ionic spectra of every stage of ionization, i.e., Si I through Si XIV. The ionization potentials range from 8 to $2673 \mathrm{eV}$, and the wavelengths of the solar identifications span the interval from $6 \AA$ to $25129 \AA$ The wide range of excitation and ionization thus represented makes silicon a suitable element for detailed study of solar models, fluxes, abundances and the like.

Okabe, H., photodissociation of HNCO in the vacuum ultraviolet; Production of $\mathrm{NCO} \mathbf{A}^{2} \Sigma$ and $\mathrm{NH}\left(\mathbf{A}^{3} \pi, \mathbf{c}^{\prime} \boldsymbol{\pi}\right) J$. Chem Phys. 53, No. 9, 3507-3515 (November 1, 1970).

Key words: Bond dissociation energy; fluorescence; HNCO; NCO $\mathrm{A}^{2} \Sigma ; \mathrm{NH} \mathrm{A}^{3} \pi, \mathrm{c}^{1} \pi ;$ photodissociation; vacuum ultraviolet.

Strong emission bands originating from $\mathrm{NCO} \mathrm{A}^{2} \Sigma$ and $\mathrm{NH}\left(\mathrm{A}^{2} \pi, c^{1} \pi\right)$ were found in the photodissociation of HNCO in the vacuum ultraviolet. In addition, weak bands coming from $\mathrm{NCO} \mathrm{B}^{2} \pi$ were observed. Threshold energies of incident photons to produce these bands were measured, from which the following bond dissociation energies and heats of formation were obtained: $\mathrm{D}(\mathrm{H}-\mathrm{NCO})=4.90 \pm 0.01 \mathrm{eV}$, $\mathrm{D}(\mathrm{HN}-\mathrm{CO})=3.38 \pm 0.1 \mathrm{eV}, \mathrm{D}(\mathrm{N}-\mathrm{CO})=2.14 \pm 0.15 \mathrm{eV}, \Delta H_{f}(\mathrm{HNCO})$ $=-1.0 \pm 0.14 \mathrm{eV}, \Delta H_{f}(\mathrm{NCO})=1.6 \pm 0.15 \mathrm{eV}$. The emission spectrum, NCO $\mathrm{A}^{2} \Sigma \rightarrow \mathrm{X}^{2} \pi$, produced from the photolysis at the Xe and $\mathrm{Kr}$ lines shows that $\mathrm{NCO} \mathrm{A}^{2} \Sigma$ is highly excited in bending vibration, indicating that the upper state of $\mathrm{HNCO}$ from which $\mathrm{NCO} \mathrm{A}^{2} \Sigma$ predissociates, has a bent NCO configuration. NH $c^{1} \pi$ formed is highly rotationally excited. Experimental evidence is presented to show that $\mathrm{NH}^{3} \pi$ is produced primarily by a secondary process, $\mathrm{CO} a^{3} \pi+\mathrm{NH} \mathrm{X} \mathrm{X}^{3} \Sigma \rightarrow \mathrm{CO} \mathrm{X}^{1} \Sigma+\mathrm{NH} \mathrm{A}^{3} \pi$. Primary processes in the near ultraviolet photolysis of HNCO are discussed on the basis of bond dissociation energies obtained in the present work.

Parker, H. S., Harding, C. A., Vapor growth of $\mathbf{A l}_{2} \mathbf{O}_{3}$ bicrystals, J. Am. Ceram. Soc. 53, No. 11, 583-585 (November 11, 1970).

Key words: Aluminum oxide; bicrystals; crystal growth; vapor crystal growth.

Bicrystals containing symmetrical tilt boundaries with either the $[10 \overline{10}]$ or the $[11 \overline{2} 0]$ direction as the rotation axis have been grown at a temperature of $1740^{\circ} \mathrm{C}$ by a vapor growth technique utilizing the reaction:

$$
2 \mathrm{AlCl}_{3}(\mathrm{~g})+3 \mathrm{CO}_{2}(\mathrm{~g})+3 \mathrm{H}_{2}(\mathrm{~g}) \rightarrow \mathrm{Al}_{2} \mathrm{O}_{3}(\mathrm{~s})+6 \mathrm{HCl}(\mathrm{g})+3 \mathrm{CO}(\mathrm{g}) .
$$

Specimens as large as $3 \times 5 \times 20 \mathrm{~mm}$ have been produced in a 24 hour growth period. Chemical purity of the specimens is very high, with less than $0.1 \mathrm{ppm}$ total cation impurities as determined by activation analysis.

Parker, R. L., Crystal growth mechanisms: Energeties, kineties, and transport, Solid State Phys. 25, 151-299 (Academic Press, Inc., New York, N.Y., 1970).

Key words: Crystal; crystal growth; crystallization; growth mechanisms; morphological stability; nucleation; phase changes; transport processes.

A review is given of the mechanisms of crystal growth. Subjects discussed include thermodynamics and statistical mechanics of phase changes; the mathematical physics of crystal growth trnasport processes; nucleation; interface structure and interface kinetics; morphological stability; impurity effects; and fluid flow effects in crystallization. Particularly emphasized are the results of quantitative studies, both theoretical and experimental, on well defined systems. Experimental techniques and apparatus are not covered, nor are solid-solid transformations or the crystallization of polymers.

Pella, P. A., DeVoe, J. R., The determination of tin in copperbase alloys by Mössbauer spectrometry, Anal. Chem. 42, No. 14, 1833-1835 (December 1970).

Key words: Alloys; analysis; internal standard; Mössbauer spectrometry; stannic oxide.

The Mössbauer spectrometric method was applied to the determination of tin in NBS SRM copper-base alloys. After converting the $\mathrm{Sn}$ in the alloy to $\mathrm{SnO}_{2}$ the absorption intensity of $\mathrm{SnO}_{2}$ was measured versus $\beta$-Sn as an internal standard absorber. Stannic oxide was obtained by the dissolution of the alloys in nitric acid. This produces a precipitate contaminated by the coprecipitation of a number of elements. Through the use of an appropriate standard, it was shown that the Mössbauer technique can measure the $\mathrm{SnO}_{2}$ concentration without interference. The results for alloys $52 \mathrm{c}, 184$, and $37 \mathrm{e}$ were $7.82 \pm .05 \%(7.85), 6.31 \pm .06 \%(6.38)$, and $0.99 \pm .01 \%(1.00)$, respectively, where the uncertainties are the relative standard deviation of the mean values and the numbers in parentheses are the NBS certified values.

Perloff, A., The crystal structure of sodium hexamolybdochromate(III) octahydrate, $\mathrm{Na}_{3}\left(\mathrm{CrMo}_{6} \mathrm{O}_{24} \mathrm{H}_{6}\right), \quad \mathbf{8} \mathrm{H}_{2} \mathrm{O}, \quad$ Inorganic Chem. 9, No. 10, 2228-2239 (1970).

Key words: Heteropolymolybdate; x-ray crystal structure.

The detailed structure of the heteropoly salt $\mathrm{Na}_{3}\left(\mathrm{CrMo}_{6} \mathrm{O}_{24} \mathrm{H}_{6}\right)$ $\cdot 8 \mathrm{H}_{2} \mathrm{O}$ has been determined using three-dimensional $\mathrm{x}$-ray diffraction data. The crystals are triclinic, space group $\mathrm{P} \overline{\mathrm{l}}$, with cell dimensions $a=10.9080, b=10.9807, c=6.4679 \mathrm{~A}, \alpha=107.594^{\circ}$, $\beta=84.438^{\circ}$ and $\gamma=112.465^{\circ}$. There is one formula unit per unit cell. The anion has the same structure as the $\left(\mathrm{TeMo}_{6} \mathrm{O}_{24}\right)^{-6}$ anion with excellent agreement of comparable bond distances. Charge 
balance and hydrogen bonding arguments suggest that the anion's hydrogen atoms are bonded to the oxygen atoms which are coordinated to the $\mathrm{Cr}$ atom. The anions are linked together through sodium octahedra and hydrogen bonding. No hydrogen atoms could be located directly, but a reasonable hydrogen bonding scheme was inferred from short oxygen-oxygen distances. Final refinement by least-squares analysis with anisotropic temperature factors resulted in an $R$ value of $3.3 \%$.

Prydz, R., Straty, G. C., Timmerhaus, K. D., Properties of fluorine along the vapor-liquid coexistence boundary, J. Chem. Phys. 53, No. 6, 2359-2363 (September 15, 1970).

Key words: Critical point; fluorine; latent heats of vaporization; saturated liquid densities; saturated vapor densities; vapor pressure.

Saturated liquid and vapor data from the triple point to the critical point are reported. These data were derived from experimental PVT isochores and a vapor pressure equation. The critical point was extracted by fitting an equation to all experimental data within 20 percent of the critical temperature. Separate equations, each constrained to the new critical point, were fitted to the saturated liquid and vapor data. Latent heats of vaporization were calculated from the Clapeyron equation and compared to other published values. A vapor pressure equation based on the IPTS 1968 temperature scale is reported.

Raveche, H. J., Mountain, R. D., Three body correlations in simple dense fluids, J. Chem. Phys. 53, No. 8, 3101-3107 (October 15,1970 )

Key words: Neutron diffraction; percus-Yevick equation; simple liquids; superposition approximation; three body correlation function; $\mathrm{x}$-ray diffraction; radial distribution function.

Correlations between triples of molecules in simple fluids at thermodynamic equilibrium are studied through their contribution to the isothermal density derivative of the pair probability density. Explicit computations are performed to indicate the role of the triplet function in accounting for the structure of the density derivative of the radial distribution function. The results imply that contributions from triplet correlations are in general quite appreciable. Various consequences of the results are discussed and the procedure is examined in general as a method for studying correlations between triples of molecules in simple fluids.

Risley, A. S., Allan, D. W., Peters, H. E., Johnson, E. H., Vessot, R. F. C., Levine, M., Gray, J. E., Shoaf, J. H., Machlan, H. E., and Glaze, D. J., Stability characteristics of some atomic frequency standards (Summary), Proc. Conf. Precision Electromagnetic Measurements, National Bureau of Standards, Boulder, Colo., June 2-5, 1970, CPEM Digest, pp. 74-75 (1970).

Key words: Allan variance; cesium beam; frequency stability; hydrogen maser; NBS frequency standard; time scale.

Over a period of three and one-half months a frequency stability comparison was made between seven commercial cesium beams and a NASA (Goddard Space Flight Center) hydrogen maser. Two of the prime purposes in bringing the NASA maser to Boulder were: (1) to determine the general effect upon the NBS Time Scale of including the $\mathrm{H}$ maser as a part and (2) to determine the stability of the $\mathrm{H}$ maser for sampling times $\tau$ longer than $10^{3}$ seconds.

Two of the major results of the time scale and NASA maser comparison were, first, $\sigma_{y}(N=2, T=\tau, \tau=1$ day) of the time scaleincluding the maser-was less than $1 \times 10^{-13}$, where $y \equiv \delta \nu / \nu$. Second, the inclusion of the maser in the time scale improved the precision about 30 percent.

Another hydrogen maser (from the Smithsonian Astrophysical Observatory) and a new four-foot long cesium beam (NBS-X4) were used along with the NASA maser to measure stabilities $\sigma_{y}(N=2, T=\tau, \tau)(\tau \approx 1$ day). The stabilities for both masers and for NBS-X4 were better than one part in $10^{13}$.

Robertson, B., Quantum statistical mechanical derivation of generalized hydrodynamic equations, J. Math. Phys. 11, No. 8, 2482-2488 (August 1970).
Key words: Conservation equations; equation of motion; hydrodynamic equations; quantum fluids; quantum mechanical density distribution; quantum statistical mechanics.

Exact differential conservation equations are derived from the mass, momentum, and energy density operators for a one-component simple fluid of Bose or Fermi particles with arbitrary pairwise interactions. These equations are used in a statistical mechanical derivation of exact equations of motion for the expectations of these operators. The equations of motion are coupled to the exact equations relating the local temperature, chemical potential, and fluid velocity to these expectations. The coupled equations are closed in the sense that the expectations and their thermodynamic conjugates listed above are the only unknowns, although some of the dependence in the equations on the conjugates is expressed only implicitly. The equations of motion are memory-retaining nonlocal generalizations of the classical hydrodynamic equations and apply to a normal fluid arbitrarily far from equalibrium. The formalism is not carried as far as has the corresponding classical formalism.

Roth, R. S., Waring, J. L., Parker, H. S., Effect of oxide additions on the polymorphism of tantalum pentoxide. IV. The system $\mathbf{T a}_{2} \mathbf{O}_{5}-\mathbf{T} \mathbf{a}_{2} \mathbf{W} \mathbf{O}_{8}$, J. Solid State Chem. 2, 445-461 (1970).

Key words: Melting points; phase equilibria; system tantalum oxide-tantalum tungstate.

The low temperature form of pure $\mathrm{Ta}_{2} \mathrm{O}_{5}$ has been found to exist in two slightly different modifications. The lowest temperature form has a $b$ axis multiplicity of 14 whereas the highest temperatures form (about $1350{ }^{\circ} \mathrm{C}$ ) has a multiplicity of 11 . At intermediate temperatures an "infinite" number of at least partially ordered sequences of these two modifications exist in equilibrium.

The addition of $\mathrm{WO}_{3}$ to $\mathrm{Ta}_{2} \mathrm{O}_{5}$ causes the "stabilization" of an "infinite" number of phases similar in structure to the low temperature form of $\mathrm{Ta}_{2} \mathrm{O}_{5}$. From just less than $10 \mathrm{~mol} \% \mathrm{WO}_{3}$ to the last compound in the series at $26 \frac{2}{3}$ mol $\% \mathrm{WO}_{3}$ these phases are in equilibrium with liquid and the high temperature structure type of pure $\mathrm{Ta}_{2} \mathrm{O}_{5}$ is eliminated from the phase diagram. One compound, $14 \mathrm{Ta}_{2} \mathrm{O}_{5} \cdot 2 \mathrm{WO}_{3}$ with a multiplicity of 8 , was found to melt congruently at about $1815{ }^{\circ} \mathrm{C}$ and the last phase, $11 \mathrm{Ta}_{2} \mathrm{O}_{5} \cdot 4 \mathrm{WO} \mathrm{W}_{3}$ with a multiplicity of 13 , melts incongruently at about $1605{ }^{\circ} \mathrm{C}$.

Rush, J. J., Schroeder, L. W., Melveger, A. J., Infrared and Raman spectra of sodium bifluoride: Isotope dilution studies, Chem. Phys. Letters 6, No. 5, 533-536 (September 1, 1970).

Key words: Bending vibration; hydrogen bond; infrared; isotope dilution; lattice mode; raman; sodium; bifluoride; stretching vibration.

IR and Raman spectra for sodium bifluoride containing $2 \%, 11 \%$, and $\sim 80 \% \mathrm{DF}_{2}^{-}$are presented. Changes in the $\mathrm{DF}_{2}^{-} \nu_{2}$ and $\nu_{3}$ IR bands with deuteration are related to coupling between like oscillators or states. Raman assignments are made for the $\mathrm{HF}_{2}^{-} \nu_{1}$ and lattice liberational $\left(\mathrm{E}_{q}\right)$ modes.

Schneider, S. J., Cooperative determination of the melting point of alumina, J. Pure Appl. Chem. 21, No. 1, 117-122 (1970).

Key words: $\mathrm{Al}_{2} \mathrm{O}_{3}$; alumina; IUPAC; melting point of $\mathrm{Al}_{2} \mathrm{O}_{3}$; melting point standards.

A task force on secondary temperature standards, sponsored by the Commission on High Temperatures and Refractories, International Union of Pure and Applied Chemistry, has undertaken a program to investigate various inorganic non-metallic substances for use as high temperature reference materials. As part of this program a cooperative determination of the melting point of $\mathrm{Al}_{2} \mathrm{O}_{3}$ (alumina) was conducted by the task force. In all, nine scientific groups, representing seven countries contributed experimental data. All work was performed utilizing a common supply of $\mathrm{Al}_{2} \mathrm{O}_{3}$ of nominal $99.9 \%$ purity. Experimental techniques varied depending upon the individual investigator. The value for the alumina point, as recommended by the task force is $2054{ }^{\circ} \mathrm{C} \pm 6$ (IPTS 1968). 
Sengers, J. M. H. L., Scaling predictions for thermodynamic anomalies near the gas-liquid critical point, Ind. Eng. Chem. Fundamentals, 9, No. 3, 470-480 (November 9, 1970).

Key words: Argon; carbon dioxide; classical equation; critical exponents; critical point; coexistence curve; lattice gas; power laws; rectilinear diameter; steam; thermodynamic anomalies; vapor pressure.

Most classical (or van der Waals type) equations of state of fluids show characteristic critical thermodynamic anomalies and symmetries that are common to them all. These characteristics are deduced from the Taylor expansion, in density and temperature, of these equations at the critical point. It is shown that the anomalies found experimentally are of a different nature, more similar to those predicted by the Ising model for the lattice gas. Consequences for data correlations in the critical region are discussed and examples of such correlations are presented.

Simmons, J. H., Macedo, P. B., Viscous relaxation above the liquid-liquid phase transition of some oxide mixtures, J. Chem. Phys. 53, No. 7, 2914-2922 (October 1, 1970).

Key words: Immiscibility; oxide glasses; relaxation times; supercritical fluctuations; ultrasonic spectroscopy; viscous relaxation.

The viscosity anomaly observed above the critical points of a series of sodium-borosilicate melts is investigated by ultrasonic shear relaxation spectroscopy. The measurements were conducted at frequencies between 3 and $25 \mathrm{MHz}$ and temperatures between $800{ }^{\circ} \mathrm{C}$ and $1300{ }^{\circ} \mathrm{C}$. The samples tested have different critical temperatures as a result of small doping additions to the critical composition of the immiscibility surface in the phase diagram of the sodium-borosilicate system. This permitted measurement over a wide range of reduced temperatures. Analysis of the data yields the three structural relaxation parameters which are: the in stantaneous shear modulus, the most probable relaxation time, and the width of the distribution of relaxation times. These param eters are related to the viscosity. It is found that while the instan taneous modulus and the most probable relaxation time appear unaffected by the presence of supercritical composition fluctua tions, the width of the spectrum of relaxation times broadens drastically as the critical temperature is approached from above. Similar and parallel curves are formed from the widths of the distributions of relaxation times of all four samples when the tem perature is properly normalized by the critical temperature. The general behavior of these curves exhibits the three reduced tem perature regions predicted in a previously published topological model. The supercritical broadening of the width of the distribution of relaxation times accounts for the observed viscosity anomaly.

Spencer, L. V., Stanley, W., On neutron penetration in duet systems with large cross sections, Proc. Special Sessions on Gamma-Ray Production and Transport and on Civil Defense Shielding, American Nuclear Society, pp. 199-221 (August 1969).

Key words: Albedo; Civil Defense; ducts; nuclear weapons data; neutron penetration; structure shielding.

Neutron albedo data for non-hydrogenous wall materials is insensitive to variations of the total cross section with energy and, to some extent, to details of cross section angular distributions. As a result, it makes sense to explore models in which the differential albedo is factored into energy dependent and direction dependent parts. This approximation results in a factoring of the transport equation for large enclosures into two parts, a one-velocity type of problem and a general problem of determining spectra for different orders of reflection. We extend this approach further to identify suitable parameters in terms of which data for elementary types of enclosure can be applied to configurations. These parameters are given, the procedure for combining duct sections is sketched, and calculations for a three-legged duct are compared with Monte Carlo data for such a duct.

Swanson, H. E., McMurdie, H. F., Morris, M. C., Evans, E. H., Standard x-ray diffraction powder patterns, Nat. Bur. Stand. (U.S.), Monogr. 25-Section 8, 171 pages (Sept. 1970) \$1.50, SD Catalog No. C13.44:25/sec. 8.
Key words: Crystal structure; integrated intensities; lattice constants; peak intensities; powder patterns; reference intensities; standard; $x$-ray diffraction.

Standard x-ray diffraction patterns are presented for 81 substances. Fifty-three of these patterns represent experimental data and 28 are calculated. The experimental x-ray powder diffraction patterns were obtained with x-ray diffractometer, using samples of high purity. All d-values were assigned Miller indices determined by comparison with computed interplanar spacings consistent with space group extinctions. The densities and lattice constants were calculated, and the refractive indices were measured whenever possible. The calculated $x$-ray power diffraction patterns were computed from published crystal structure data. Both peak height and integrated intensities were reported for the calculated patterns.

Tsai, D. H., Bullough, R., Perrin, R. C., Molecular dynamical studies of the motion of point defects in a crystalline lattice, Proc. Phys. Soc. (London) C: Solid St. Phys. 3, 2022-2036 (1970)

Key words: Atomic diffusion; defect motion; interstitial motion; jump frequency; lattice model; migration energy; migration entropy; molecular dynamics; random-walk theory; vacancy motion.

Molecular dynamical calculations have been employed to study the motion of interstitial and vacancy defects in a bec lattice with an interaction potential which simulated that of $\alpha$-iron. Our purpose was to investigate the effects of lattice thermal oscillations on the motion and the interactions of these defects, and to study the random-walk problem of defect motion on an atomic scale. The dynamical results showed that the temperature dependence of the defect jump frequency was in general agreement with the atomic theory of diffusion. From this temperature dependence, the energy and the entropy of defect migration were obtained. For interstitial motion, the migration energy from the dynamical model $(0.23 \mathrm{eV})$ was the same as that from static calculations. For vacancy motion, the migration energy from the dynamical model $(0.42 \mathrm{eV})$ was lower than that from static calculations. Possible reasons for this difference are discussed.

Wasik, S. P., Tsang, W., Determination of trace amounts of contaminants in water by isotope dilution gas chromatography, Anal. Chem. 42, No. 13, 1649-1651 (November 1970).

Key words: Chromatics; gas chromatography; isotope dilution; olefins; and water analysis.

An isotope dilution technique using gas liquid chromatography is proposed for the quantitative analysis of trace organic pollutants in water supplies. The method was demonstrated using benzene with predeuterated benzene as the isotope with both substances in the p.p. B. concentration range. Columns with aqueous silver nitrate as the stationary phase were used to determine the ratio of the concentration of benzene to predeuterated benzene.

Wasik, S. P., Tsang, W., Gas-liquid chromatography separations of hydrocarbons using columns with aqueous solutions of complexing metal ions as stationary phases, Anal. Chem. 42, No. 13, 1648-1649 (November 1970).

Key words: Aliphatics; aromatics; complexing effects; gas chromatography; group separation; mercuric nitrate; olefins; silver nitrate; surface effect.

Interesting hydrocarbon selectivity was obtained in gas-liquid chromatography using aqueous solutions of $\mathrm{Ag}^{+}$and $\mathrm{Hg}^{++}$ions as the stationary phases. This was achieved by taking advantage of the complexing properties of these ions and the large surface effect of the solution. Columns may be designed to be specific for certain hydrocarbon groups using this technique.

Waxman, M., Hastings, J. R., Chen, W. T., Nonlinear statistical analysis of Burnett PVT data, Proc. 5th Symp. Thermophysical Properties, Boston, Massachusetts, September 30-October 2, 1970 , C. F. Bonilla, Ed., pp. 248-261 (Americal Society of Mechanical Engineers, New York, N.Y., 1970). 
Key words: Burnett; gas; statistical; model dependency; nonlinear; PVT.

The Burnett method, which requires only temperature and isothermal pressure measurements made before and after stepwise expansions between two volumes, is being used extensively for determining gas compressibilities and density or pressure viria coefficients. The measurements are simple, but the data reduction involves rather formidable nonlinear statistical analysis of relationships containing the compressibility factor, defined as a density or a pressure virial expansion, and optional constants. In this paper we compare the results of analyzing different Burnett relationships with independent parameters and no restrictions on the data, using two nonlinear statistical methods, due to Gauss and to Deming, and argon data at $25{ }^{\circ} \mathrm{C}$ for pressures up to 250 atm. We discuss the computational difficulties and present background information on the experimental and analytical methods.

Weir, C. E., Block, S., Piermarini, G. J., Compressibility of inorganic azides, J. Chem. Phys. 53, No. 1, 4265-4269 (December 1970).

Key words: Anisotropic compressibility; azides; compressibility; high pressure; single crystal; x-ray diffraction.

The compressibility of $\alpha$ lead azide, $\beta$ lead azide, barium azide, potassium azide, sodium azide and thallium azide have been meas ured by single crystal x-ray diffraction techniques using the diamond anvil cell. Both the anisotropic and volume compressibilities are reported. The pressures were determined by measurements at the known freezing points of $n$-hexane and ethanol. A phase transition occurs in thallium azide at a pressure between the freezing points of chloroform (5390 bars) and $n$-decane (2990 bars). Pressure temperature observations in the diamond cell of lead azide were carried out to $300{ }^{\circ} \mathrm{C}$ and approximately $30 \mathrm{kbar}$. No phase transitions were observed. Radiation damage to azide crystals under high pressures is reduced significantly.

Weisman, H. M., Sherwood, G. B., Annotated accession list of data compilations of the office of standard reference data, Nat. Bur. Stand. (U.S.), Tech. Note. 554, 196 pages (Sept. 1970) $\$ 1.50$, SD Catalog No. C13.46:554.

Key words: Accession list; atomic and molecular properties; chemical kinetics; colloid and surface properties; fundamental particles properties; general collections; mechanical properties; nuclear properties; solid state properties; thermodynamic and transport properties.

The National Bureau of Standards, Office of Standard Reference Data has attempted to acquire all significant reference data compilations on a worldwide basis. This publication lists the collection of documents so acquired, together with their abstracts. The documents are organized in the following categories: General Collections, Nuclear Properties (including Fundamental Particles Properties) Atomic and Molecular Properties, Solid State Properties, Chemical Kinetics, Colloid and Surface Properties, Mechanical Properties, and Thermodynamic and Transport Properties. Sources of availability for the listed publications are also provided.

Wexler, A., Measurement of humidity in the free atmosphere near surface of the earth (Proc. American Meteorological Society Symp. on Meteorological Observations and Instrumentations, Washington, D.C., February 10-14, 1969) Meteorological Monographs 11, No. 33, 262-282 (October 1970).

Key words: Dew point; hygrometry; meteorology; moisture measurement in gases; psychrometry.

This paper is a survey of the state-of-the-art in hygrometry as applied to humidity measurement in gases near atmospheric pressure. Although not indicated specifically, many of the methods discussed are applicable to humidity measurements in gases at pressures higher or lower than atmospheric. Hygrometric methods, techniques and instruments are divided arbitrarily into the following six classes: (1) methods dependent upon the total removal of water vapor from a moist gas, (2) methods dependent upon the addition of water vapor to produce a saturated gas, (3) methods dependent upon the reversible sorption of water vapor by a sensor, (4) methods dependent upon the measurement of a physical property of a gas, (5) condensation methods, that is, methods dependent upon the liquid-vapor or the solid-vapor equilibrium state of the water substance, and (6) methods dependent upon chemical reactions and procedures. The methods of each class are further subdivided by principles of operation and are examined to assess such characteristics as range, accuracy, sensitivity, speed of response, effect of environmental conditions, and limitations in behavior and utility.

Wiederhorn, S. M., Bolz, L. H., Stress corrosion and static fatigue of glass, J. Am. Ceram. Soc. 53, No. 10, 543-548 (October 1970).

Key words: Crack propagation; fracture; glass; static fatigue; stress corrosion.

Stress corrosion cracking of six glasses was studied using fracture mechanics techniques. Crack velocities in water were measured as a function of applied stress intensity factor and temperature. Apparent activation energies for crack motion were obtained. Data were consistent with the universal fatigue curve for static fatigue of glass and it was found that the universal fatigue curves depended on glass composition. Of the glass compositions tested, silica glass exhibited most resistance to static fatigue followed by aluminosilicate and borosilicate glass. Sodium was found to be detrimental to stress corrosion resistance. The crack velocity data could be explained by the Charles and Hillig theory of stress corrosion and is probable that stress corrosion of glass is normally caused and controlled by a chemical reaction between the glass and water.

Wiederhorn, S. M., Townsend, P. R., Crack healing in glass, J. Am. Ceram. Soc. 53, No. 9, 486-489 (September 1970).

Key words: Crack healing; fracture; glass; strength; surface.

This paper discusses strength recovery in glass containing cracks that were observed to close spontaneously. Measurements of bonding across the surfaces of closed cracks were made on soda-lime glass, using fracture mechanics techniques. Approximately 80 percent strength recovery was observed on cracks formed by mechanical shock, while approximately 20 percent strength recovery was observed on cracks that closed after being held open to the atmosphere for several minutes. The high strength recovery during closure of cracks formed by mechanical shock is attributed to the very active nature of the surface formed during fracture. Reaction with air or dry nitrogen is believed to reduce the activity of the fracture surfaces because of oxygen and water adsorption.

\section{Other NBS Publications}

J. Res. Nat. Bur. Stand. (U.S.), 75C (Eng. \& Instr.), No. 1 (January-March 1971), SD Catalog No. Ci13.22/sec.C:75/1

Rotary-vane attenuator with an optical readout. W. E. Little, W. Larson, and B. J. Kinder.

Design and operational characteristics of a high-speed (millisecond) system for measurement of properties at high temperature. A. Cezairliyan.

New method of determining residual thiosulfate in processed photographic film. C. I. Pope.

Scanning densitometer for continuous recording of spectral transmission density at low spatial contrast. W. L. McLaughlin, M. Rosenstein, E. Hussmann, and J. J. Lantz, Jr.

Rotating optical attenuator for the generation of subsecond duration sawtooth shape radiance pulses. A. Cezairliyan.

Sound speed measurements in solids; absolute accuracy of an improved transient pulse method. T. M. Proctor, Jr.

Seismic response of infrasonic microphones. A. J. Bedard, Jr.

Publications of the National Bureau of Standards.

Achenbach, P. R., Building research at the National Bureau of Standards, Nat. Bur. Stand. (U.S.), Bldg. Sci. Ser. 0, 59 pages (Oct. 1970) 60 cents, SD Catalog No. C13.29/2:0. 
Acquista, N., Abramowitz, S., Structure of the alkali hydroxides. V. The infrared spectra of matrix-isolated $\mathrm{RbOH}, \mathrm{RbOD}, \mathrm{NaOH}$, and NaOD, J. Chem. Phys. 51, No. 7, 2911-2914 (October 1, 1969).

Ahearn, A. J., Homogeneity of ion sensitive emulsions and precision of ion beam measurements in spark source mass spectrometry, (Summary), Proc. 16th Annual Conf. on Mass Spectrometry and Allied Topics, May 12-17, 1968, Pittsburgh, Pa., sponsored by ASTM Committee E14, pp. 273-276 (1968).

Andrews, J. R., Improved bias supply for tunnel-diode picosecond pulse generators, IEEE Trans. Instr. Meas. IM-19, No. 3, 171-175 (Aug. 1970).

Armstrong, R. W., Waters, H. P., Testing programs and research on restraint systems, Proc. Intern. Engineering Congress, Detroit, Michigan, January 13-17, 1969, Report No. 690247, pp. 1-48 (Society of Automotive Engineers, New York, N.Y., 1969); Abstract in SAE Trans. 78, 102 (1969).

Armstrong, R. W., Waters, H. P., Stapp, J. P., Human muscular restraint during sled deceleration, Proc. 1968 Stapp Car Crash Conf., October 22-23, 1968, Detroit, Michigan, Report No. 680793, pp. 440-462 (Society of Automotive Engineers, New York, N.Y., 1969); Abstract in SAE Trans. 77, 179 (1969).

Arthur, M. G., Impulse spectral intensity calibration at the National Bureau of Standards, Proc. Electromagnetic Compatibility Symp., Ft. Monmouth, N.J., June 19-20, 1969, pp. 133-138 (U.S. Army Electronics Command Hdq., Ft. Monmouth, N.J., 1969).

Ausloss, P., Lias, S. G., Carbonium ions in radiation chemistry. Reactions of $t$-butyl ions with hydrocarbons, J. Am. Chem. Soc. 92, 5037-5045 (1970).

Bagg, T. C., Factors dictating characteristics of systems utilizing microforms, J. Micrographs 3, No. 3, 143-146 (1970); ABFM Format 1, No. 2, 1-2 (May 1970).

Baird, R. C., Recent theoretical and experimental results of the determination of antenna pattern and gain from near-field measurements (Summary), Proc. Conf. Precision Electromagnetic Measurements, National Bureau of Standards, Boulder, Colo. June 2-5, 1970, CPEM Digest, p. 60 (1970).

Baird, R. C., Newell, A. C., Wacker, P. F., Kerns, D. M., Recent experimental results in near-field antenna measurements, Electronics Letters 6, No. 11, 349-351 (May 28, 1969).

Barnes, J. A., Frequency measurement errors of passive resonators caused by frequency-modulated exciting signals, IEEE Trans. Instr. Meas. IM-19, No. 3, 147-152 (August 1970).

Barnes, J. A., Chi, A. R., Cutler, L. S., Healey, D. J., Leeson, D. B., MeGunigal, T. E., Mullen, J. A., Smith, W. L., Sydnor, R., Vessot, R. F. C., Winkler, G. M. R., Characterization of frequency stability, Nat. Bur. Stand. (U.S.), Tech. Note 394, 50 pages (Oct. 1970) 60 cents, SD Catalog No. C13.46:394

Bates, R. D., Jr., Flynn, G. W., Ronn, A. M., Laser-induced vibrational fluorescence in nitrous oxide, J. Chem. Phys. 49, No. 3, 1432-1433 (1968)

Bates, R. G., Staples, B. R., Robinson, R. A., Ionic hydration and single ion activities in unassociated chlorides at high ionic strengths, Anal. Chem. 42, No. 8, 867-871 (July 1970).

Bennett, H. S., Two-electron $F^{\prime}$ centers in the alkaline-earth oxides and in the alkali halides, Phys. Rev. B, 1, No. 4, 1702-1715 (February 15,1970 ).

Berger, M. J., Beta-ray dosimetry calculations with the use of point kernels (Proc. Symp. Medical Radionuclides: Radiation Dose and Effects, Oak Ridge Association Universities, Oak Ridge, Tennessee), Atomic Energy Commission Report CONF-691212, pp. 63-86 (June 1970).

Bergstrom, J. C., Bertozzi, W., Kowalski, S., Maruyama, X. K. Lightbody, Jr., J. W., Fivozinsky, S. P., Penner, S., Electroexcitation of the low-lying of $0^{16}$, Phys. Rev. Letters 24, No. 4, 152-155 (January 26, 1970)

Birky, M. M., Simultaneous recording of near-field and far-field patterns of lasers, Appl. Opt. 8, No. 11, 2249-2253 (November 1969).

Blaine, R. L., A statistical study of the effects of trace elements on the properties of portland cement, Proc. 5th Intern. Symp. on the Chemistry of Cements, Tokyo, Japan, October 7-11, 1968 , 3, Part 3, 86-91 (December 31, 1969).

Blandford, J. M., Gurel, L. M., Fibers and fabrics, Nat. Bur. Stand. (U.S.), Consumer Info. Series 1, 31 pages (Nov. 1970) 65 cents, SD Catalog No. C13.53:1

Blaugher, R. D., Hein, R. E., Cox, J. E., Waterstrat, R. M., Atomic ordering and superconductivity in A-15 compounds, J. Low Temp. Phys. 1, No. 6, 539-561 (December 1969)

Boyne, H. S., Hall, J. L., Barger, R. L., Bender, P. L., Ward, J. Levine, J., Faller, J., Absolute strain measurements with a 30meter vacuum interferometer, (Proc. Conf. Laser Applications in the Geosciences, Huntington Beach, Calif., June 1969), Chapter in Laser Applications in the Geosciences, J. Gauger and F. F. Hall, Jr., Ed., pp. 215-225 (Western Periodicals, North Hollywood, Calif., 1970)

Brady, R. F., Jr., Cyclic acetals of ketoses. Part III. Re-investigation of the synthesis of the isomeric Di- $O$-isopropylidene- $\beta$-D-fructopyranoses, Carbohydrate Res. 15, No. 1, 35-40 (1970).

Brenner, Tires. Their selection and care, Nat. Bur. Stand. (U.S.), Consumer Info. Series 2, 32 pages (Nov. 1970) 65 cents, SD Cata$\log$ No. C13.53:2.

Bullis, W. M., Baroody, A. J., Jr., Methods of measurement for semiconductor materials, process control, and devices, Quarterly Report-January 1 to March 31, 1970, Nat. Bur. Stand. (U.S.), Tech. Note 555, 63 pages (Sept. 1970) 65 cents, SD Catalog No. C13.46:555.

Bullis, W. M., Scace, R. I., Measurement standards for integrated circuit processing, Proc. IEEE 57, No. 9, 1639-1646 (September 1969).

Casella, R. C., Possible failure of the Pomeranchuk theoremshrinkage of the forward elastic peak, Phys. Rev. Letters 24, No. 25, 1463-1466 (June 22, 1970)

Champion, E. C., Marinenko, G., Taylor, J. K., Schmidt, W. E., Determination of submicrogram amounts of chromium by coulometric titrimetry, Anal. Chem. 42, 11, 1210-1213 (September 1970).

Chandler, R. F., Christian, R. A., Comparative evaluation of dummy performance under $-G_{x}$ impact, Proc. 13th Stapp Car Crash Conf., Boston, Mass., December 2-4, 1969, Report No. 690798, pp. 61-75 (Society of Automotive Engineers, New York, N.Y., 1970); Abstract in SAE Trans. 78, 171-172 (1969).

Clark, A. F., Childs, G. E., Wallace, G. H., Electrical resistivity of some engineering alloys at low temperatures, Cryogenics 10, No. 4, 295-305 (August 1970).

Coriell, S. R., Jackson, J. L., Probability distribution of the radius of gyration for two statistical segments, J. Chem. Phys. 53, No. 8, 3389 (October 15, 1970).

Cornog, D. Y., Cornog, J. R., Human factors engineering in the sorting and handling of mail, Proc. NATO Advanced Study Institute on "Human Factors/Ergonomics," Mondello, Sicily, September 29, 1969, pp. 1-34 (January 21, 1970).

Costrell, L., Development and current status of the standard nuclear instrument module (NIM) system, Nat. Bur. Stand. (U.S.), Tech. Note 556, 15 pages (Oct. 1970) 30 cents, SD Catalog No. C13.46:556.

Coxon, B., The conformations of 1,2:4,6-Di- $O$-benzylidene- $\alpha$-Dglucopyranose derivatives, Carbohydrates Res. 14. 9-15 (October 1970).

Crissman J. M., Passaglia, E., Eby, R. K., Colson, J. P., Crystal data on n-eicosane $\left(\mathrm{C}_{20} \mathrm{H}_{42}\right)$, J. Appl Cryst. 3, 193-195 (1970).

Cruz, J. E., Jellison, J. C., A digital technique for generating variable frequency multiphase waveforms, Rev. Sci. Instr. 41, No. 7 , 1098-1099 (July 1970).

Currie, L. A., Radioactivity monitors and accuracy in photonuclear experiments, (Summary), Trans. Am. Nucl. Soc. 13, No. 1, 59 (1970).

Davis, D. D., Transmission of time/frequency signals in the vertical interval, Proc. 108th Technical Conference and Equipment Exhibit, New York, N.Y., October 4-9, 1970, Article No. 43, p. 3 (Society of Motion Picture and Television Engineers, New York, N.Y., 1970)

Davis, D. D., Jespersen, J. L., Kamas, G., The use of television signals for time and frequency dissemination, Proc. IEEE 58 , No. 6,931-933 (June 1970).

Dickens, B., Brown, W. E., The crystal structure of calcium carbonate hexahydrate at $\sim-120^{\circ}$, Inorg. Chem. 9, No. 3, 480-486 (March 1970).

Diller, D. E., Hanley, H. J. M., Roder, H. M., The density and temperature dependence of the viscosity and thermal conductivity of dense simple fluids, Cryogenics 10, No. 4, 286-294. (August 1970).

Engen, G. F., A new method of charaterizing amplifier noise performance (Summary), Proc. Conf. Precision Electromagnetic Measurements, National Bureau of Standards, Boulder, Colo. June 2-5, 1970, CPEM Digest, pp. 20-21 (1970). 
Farrar, T. C., Pulsed and Fourier transform NMR spectroscopy, Anal. Chem. 42, No. 4, 109A-112A (April 1970).

Frederick, N. V., Scott. W. W., Jr., A simple technique for metalizing boron nitride, Rev. Sci. Instr. 40, No. 9, 1240 (September 1969).

Freeman, D. H. Zielinski, W. L., Jr., Rittner, W. F., Recognition of crosslinking in the infrared spectra of poly(styrene-divinylben zene), Proc. Ion Exchange in the Process Industries Conf., Imperial College of Science and Technology, London, England, pp. 27-30 (1970).

Gibson, B. F., O'Connell, J. S., Angular distribution of the reaction ${ }^{1} \mathrm{H}\left(d^{3}, \mathrm{He}\right)$ Physics Letters 32B, No. 5, 331-332 (August 3, 1970).

Goldman, A. J., Analysis of a capacity concept for runway and final-approach path airspace, Proc. Ion National Meeting on Air Traffic Control in the 1970's, Institute of Navigation, St. Louis, Mo., April 14-16, 1970, pp. 119-131 (1970).

Greenberg, L., Newman, M., Some results on solvable groups, Arch. Math. 21, No. 4, 349-352 (1970).

Greenfeld, S. H., Warner, E. R. Reinhart, H. W., Bibliographies on fabric flammability. Part 5. Testing and test methods, Nat. Bur. Stand. (U.S.), Tech. Note 498-2, 39 pages (Sept. 1970) 45 cents, SD Catalog No. C13.46:498-2.

Grosch, H. R. J., A view of computers from the Bureau of Standards, Input 6, No. 2, 3-7 (1970).

Halford, D., Frequency stability of quality quartz crystal oscillators: Performance and some critical applications, Proc. Intern Colloq. Chronometrie, Paris, France, September 16-20, 1969, Series A, pp. A-11-1-A-11-3 (September 1969).

Hardy, S. C., Coriell, S. R., Morphological stability of ice cylinders in aqueous solution, J. Crystal Growth 7, No. 2, 147-154 (September-October 1970).

Hayward, E., Schwartz, R. B., Murray, K. M., Comments on isospin of the $\mathrm{B}^{11}$ giant resonance, Phys. Rev. 2, No. 2, 761-762 (August 1970).

Hellwig, H., The hydrogen storage beam tube, a proposal for a new frequency standard, Metrologia 6, No. 2, 56-60, (April 1970).

Hellwig, H., Allan, D. W., Glaze, D. J., Vessot, R. F. C., Levine, M., Zitzewitz, P. W., Peters, H. E., Measurement of the unperturbed hydrogen hyperfine transition frequency (Summary), Proc. Conf. Precision Electromagnetic Measurements, National Bureau of Standards, Boulder, Colo., June 2-5, 1970, CPEM Digest, pp. 72-73 (1970).

Henderson, M., Berry, P. L., Progress of federal library automation, Drexel Library Quart. 6, Nos. 3 \& 4, 249-263 (July-October 1970).

Hiza, M. J., Duncan, A. G., A correlation for the prediction of interaction energy parameters for mixtures of small molecules, AIChE J. 16, No. 5, 733-738 (September 1970).

Hoer, C., Agy, D., A broadhurst resistive coupler, Proc. Conf. Precision Electromagnetic Measurements (Summary), National Bureau of Standards, Boulder, Colo., June 2-5, 1970, CPEM Digest, pp. 18-19(1970).

Homan, D. N., Zapf, T. L., Two-stage guarded inductive voltage division for use at $100 \mathrm{kHz}$, Proc. 1969 Instrument Society of America Conf., Houston, Texas, October 27-30, 1969, pp. 69-614 (1969).

Hudson, R. P., Conference Reports-Ultra-low temperatures 1970 , Cryogenics 10, No. 5, 445 (October 1970).

Hunt, C. M., Woolf, A. R., Comparison of some different methods for measuring particle size using microscopically calibrated glass beads, Powder Technol. 3, 9-23 (1969).

Jennings, D. A., West, E. D., A laser power meter for large beams, Rev. Sci. Instr. 41, No. 1, 565-567 (April 1970).

Johannesen, R. B., Ferretti, J. A., Harris, R. K., UEAITR: A new computer program for analysis of NMR spectra analysis of the proton spectrum of triisopropylphosphine, J. Magnetic Resonance 3, No. 1, 84-93 (July 1970).

Joiner, B. L., Laubscher, N. F., Brown, E. S., Levy, B., An author and permuted title index to selected statistical journals, Nat. Bur. Stand. (U.S.), Spec. Publ. 321, 510 pages (Sept. 1970) \$5.75, SD Catalog No. C13.10:321.

Jordan, R. H., Stark, M. C., Bunn, C. O., Donaldson, J. L., Obright, W. J., Millie, H. R., Gilsinn, J., Goldman, A. J., Horn, W. A., Systems analysis of inland consolidation centers for marine cargo, Nat. Bur. Stand. (U.S.), Tech. Note 530, 162 pages (Nov. 1970) \$1.25, SD Catalog No. C13.46:530.

Kearsley, E. A., Intrinsic errors for pressure measurements in a slot along a flow, (Proc. Fifth Intern. Congress on Rheology,
Kyoto, Japan, October 1968), Trans, Soc. Rheology 14:3, 419-424 (1970).

Kerns, D. M., Correction of near-field antenna measurements made with an arbitrary but known measuring antenna, Electronics Letters 6, No. 11, 346-347 (May 28, 1970).

Kerns, D. M., New method of gain measurement using two identica antennas, Electronics Letters 6, No. 11, 348-349 (May 28, 1970).

Kidnay, A. J., Hiza, M. J., Physical adsorption in cryogenic engineering, Cryogenics 10, No. 4, 271-277 (August 1970).

Krauss, M., Mies, F. H., Molecular-orbital calculation of the shape resonance in $\mathrm{N}_{2}^{-}$, Phys. Rev. A. 1, No. 6, 1592-1598 (June 1970).

LaFleur, P. D., Becker, D. A., Editors, Activation analysis section: Summary of activities, July 1969 to June 1970, Nat. Bur. Stand. (U.S.), Tech. Note 548, 164 pages (Dec. 1970) \$1.25, SD Catalog No. C13.46:548)

Lance, H. W., Report on metrology sessions at the 24th annual ISA conference, Appl. Opt. 9, No. 6, 1497-1498 (June 1970).

Lide, D. R., Jr., Editor, Critical evaluation of data in the physica sciences - A status report on the National Standard Reference Data System-June 1970, Nat. Bur. Stand. (U.S.), Tech. Note 553, 77 pages (Sept. 1970) 70 cents, SD Catalog No. C13.46:553.

Maki, A. G., Hexter, R. M., Resonance interactions with $\nu_{5}$ of $\mathrm{CH}_{3} \mathrm{I} ; \mathrm{A}$ method of determining $\mathrm{A}_{0}$, J. Chem. Phys. 53, No. 1 , 453-454 (July 1, 1970).

Mandel, J., Paule, R. C., Interlaboratory evaluation of a material with unequal numbers of replicates, Anal. Chem 42, No. 11 , 1194-1197 (September 1970).

Marsden, C. P., Tabulation of published data on electron devices of the U.S.S.R. Through March 1970, Nat. Bur. Stand. (U.S.), Tech. Note 526, 122 pages (Oct. 1970) \$1.25, SD Catalog No. C13.46:526. Supersedes NBS Technical Note 441 .

Marshall, R. D., Thom, H. C. S., Editors, Proceedings of technical meeting concerning wind loads on buildings and structures held at the National Bureau of Standards, Gaithersburg, Maryland, January 27-28, 1969, Nat. Bur. Stand. (U.S.), Bldg. Sci. Ser. 30, 172 pages (Nov. 1970) \$1.75, SD Catalog No. C13.29/2:30.

Martin, W. C., Kaufman, V., $4 p^{2}$ configuration in Neutral Zinc (Zn I), J. Opt. Soc. Am. 60, No. 8, 1096-1099 (August 1970).

Mather, W. G., III, Kit, B. V., Bloch, G. A., Herman, M. F., Man, his job, and the environment: A review and annotated bibliography of selected recent research on human performance, Nat. Bur. Stand. (U.S.), Spec. Publ. 319, 107 pages (Oct. 1970) \$1.00, SD Catalog No. C13.10:319.

Mazur, J., Non-self-intersecting random walks in lattices with nearest-neighbor interactions, Chapter in Stochastic Processes in Chemical Physics, pp. 261-280 (John Wiley \& Sons, Inc., New York, N.Y., 1969).

McSparron, D. A., Mohan, K., Raybold, R. C., Saunders, R. D., Zalewski, E. F., Spectroradiometry and conventional photometry an interlaboratory comparison, Nat. Bur. Stand. (U.S.), Tech. Note 559,197 pages (Nov. 1970) \$1.50, SD Catalog No. C13.46:559.

Meadow, C. T., Meadow, H. R., Organization, maintenance and search of machine files, Chapter 7 in Annual Review of Information Science and Technology, C. A. Cuadra, Ed., pp. 169-191 (Encyclopedia Britannica, Chicago, Ill., 1970).

Meijer, P. H. E., The importance of dipole-dipole interactions in ultralow temperature physics (Proc. 1970 Ultralow Temperature Symp. sponsored by Office of Naval Research and Naval Research Laboratory, April 23-24, 1970), R. A. Hein, D. U. Gubser, E. H. Takken, Ed., NRL Report 7133, pp. 123-128 (April 1970). Meinke, W. W., Analytical chemistry-a fading discipline? No! Anal. Chem. 42, 26A-38A (June 1970).

Melmed, A. J., Carroll, J. J., Some initial observations of TiNi in the field-ion microscope, Appl. Phys. Letters 17, No. 6, 247-249 (September 15, 1970).

Menis, O., Shultz, J. I., Editors, Analytical coordination chemistry section: Summary of Activities July 1969-June 1970, Nat. Bur. Stand. (U.S.), Tech. Note 544,151 pages (Sept. 1970) \$1.25, SD Catalog No. C13.46:544.

Meshkov, S., Comments on baryon spectroscopy, Proc. Hyperon Resonances Conf., Duke University, Durham, N.C., pp. 471-500 (April 24-25, 1970).

Meshkov, S., How good are symmetry predictions? Proc. Intern. Conf. on Symmetries and Quark Models, Wayne State University, Detroit, Michigan, June 18-20, 1969, Edited by Ramesh Chand, pp. 199-212 (November 1970).

Moore, C. E., Annual Report, July 1, 1968-June 30, 1969, The National Bureau of Standards, Washington, D.C., Bull. Am. Astron. Soc. 2, No. 1, 99-100(1970). 
Moore, G. A., Is quantitative metallography quantitative? Am. Soc. Testing Mater. Spec. Publ. 480, pp. 3-48 (1970).

Nargolwalla, S. S., Niewodniczanski, J., Suddueth, J. E., Experimental sensitivities for $3-\mathrm{MeV}$ neutron activation analysis, J. Radioanal. Chem. 5, 403-423 (1970).

Nargolwalla, S. S., Suddueth, J. E., Rook, H. L., Determination of pulse pileup and nuclear interferences in $14-\mathrm{MeV}$ neutron activation analysis for trace oxygen (Summary), Trans. Am. Nucl. Soc. 13, No. 1, 78-79 (1970).

Neill, A. H., Jr., High-energy light detector for use with pulsed ruby and glass lasers, Appl. Opt. 9, No. 10, 2392-2393 (October 1970).

Newton, C. J., Ruff, A. W., Jr., X-ray study of annealing in plastically deformed Ag-Sn alloys, Met. Trans. 1, No. 10, 2833-2838(October 1970).

Oberholtzer, B. L., Publications of the National Bureau of Standards 1968-1969, Nat. Bur. Stand. (U.S.), Spec. Publ. 305, Suppl. 1, 497 pages (December 1970) \$4.50, SD Catalog No. C13.10:305 Suppl.

O'Connell, J. S., Electron scattering sum rule for ${ }^{2} \mathrm{H},{ }^{3} \mathrm{H},{ }^{3} \mathrm{He}$ and ${ }^{4} \mathrm{He}$, Physics Letters 32B, No. 5, 323-325 (August 3, 1970).

Otoshi, T. Y., Stelzried, C. T., Yates, B. C., Beatty, R. W., Com parisons of waveguide losses calibrated by the DC potentiometer, AC ratio transformer, and reflectometer techniques, IEEE Trans. Microwave Theory Tech. MTT-18, No. 7, 406-409 (July 1970)

Peavy, S. T., Varner, R. N., Bremer, S. G., A systems programmer's guide for implementing OMNITAB II, Nat. Bur. Stand. (U.S.), Tech. Note 550, 43 pages (Nov. 1970) 50 cents, SD Catalog No. C13.46:550.

Perel, J., Deslattes, R. D., Extended fine-structure in x-ray absorption spectra of certain perovskites, Phys. Rev. B. 2, No. 5, 1317 1323 (September 1, 1970).

Plante, E. R., Paule, R. C., Explanation of the $\mathrm{H}^{\circ}$ vs $\mathrm{S}^{\circ}$ correlation, J. Chem. Phys. 53, No. 9, 3770-3771 (November 1, 1970).

Plitt, K. F., Adhesives for everyday use, Nat. Bur. Stand. (U.S.) Consumer Info. Series 3, 17 pages (Nov. 1970) 40 cents, SD Cata$\log$ No. C13.53:3.

Powell, C. J., Comparison of optical data for sodium and potassium with interband-transition absorption theory, Optics Commun. 2, No. 2, 87-89 (July 1970).

Powell, C. J., Validity of inelastic-electron-scattering data in determining the metallic or insulating properties of adsorbed atomic layers, Phys. Rev. B-1, No. 10, 4191-4192 (May 1970).

Radebaugh, R., Siegwarth, J. D., Theory of $\mathrm{He}^{3}-\mathrm{He}^{4}$ dilution refrigerators (Proc. 1970 Ultralow Temperature Symp., Washington, D.C., April 23-24, 1970; NRL Report 7133) pp. 63-81 (1970).

Radlinski, R. W., Price, J. I., The brake pedal force capability of adult females, Nat. Bur. Stand. (U.S.), Tech. Note 557, 25 pages (Oct. 1970) 35 cents, SD Catalog No. C13.46:557.

Rasmussen, A. L., Laser energy and power measurement with a double reflecting plate calorimeter, Rev. Sci. Instr. 41, No. 10 , 1479-1484 (October 1970).

Reader, J., Sugar, J., Ionization energies of Ce I and Gd I, J. Opt. Soc. Am. Letter to Editor 60, No. 10, 1421-1422 (October 1970).

Santone, L. C., Berlin, G., Location of fire stations, Proc. WORC Symp. Systems Analysis for Social Problems, National Bureau of Standards, Gaithersburg, Maryland, May 26-28, 1969, pp. 79-91 (December 3, 1970).

Saylor, C. P., Broken Virtue, Capital Chem. 20, No. 6, 137-140 (September 1970).

Schooley, J. F., Soulen, R. J., Koonce, C. S., Surface potential barrier in $\mathrm{SrTiO}_{3}$. Solid State Commun. 7, 1077-1079 (1969).

Selby, M. C., The Bolovac and its applications (Summary), Proc. Conf. Precision Electromagnetic Measurements, National Bureau of Standards, Boulder, Colo., June 2-5, 1970, CPEM Digest, pp. 14-15 (1970).

Shields, W. R., Editor, Analytical mass spectrometry section: Summary of activities July 1969 to June 1970, Nat. Bur. Stand. (U.S.), Tech. Note 546, 120 pages (Nov. 1970) \$1.25, SD Catalog No. C13.46:546.
Sieck, L. W., Searles, S. K., High pressure photoionization mass spectrometry. Reactions of alkane and cycloalkane molecular ions with water vapor at thermal kinetic energies, J. Chem. Phys. 53, No. 1, 2601-2604 (October 1, 1970).

Sieck, L. W., Searles, S. K., Rebbert, R. E., Ausloos, P., Reactivity of the cyclohexane Ion, J. Phys. Chem. 74, No. 21, 3829-3831 (October 15, 1970).

Simmons, J. H., Napolitano, A., Macedo, P. B., Supercritical viscosity anomaly in oxide mixtures, J. Chem. Phys. 53, No. 3, 1165-1170 (August 1, 1970).

Sitterly, C. M., The present state of Atomic Spectra, J. Opt. Pura Aplicada 11, No. 3, 103-113 (1969).

Sleater, G. A., Freeman, D. H., Rapid desorption of chromium (III) from cation exchanger with hydrogen peroxide solutions, Anal. Chem. 42, No. 13, 1666-1668 (November 1970).

Staveley, L. A. K., Hard-sphere model applied to the solubility of gases in low-boiling liquids, J. Chem. Phys. 53, No. 8, 3136-3138 (October 15, 1970).

Stein, P. G., Image-analyzing microscopes, Anal. Chem. 42, No. 13, 103A-107A (November 1970).

Stevens, M. E., Introduction to the Special Issue on Optical Character Recognition (OCR), Pattern Recog. 2, No. 3, 147-150 (September 1970).

Stevens, M. E., Selected R \& D requirements in the computer and information sciences, Proc. Fall Joint Computer Cong., Houston, Texas, November 17-19, 1970, pp. 159-168 (AFIPS Press, Montvale, N.J., 1970).

Stevenson, D. R., Technical Standards Coordinator, Rigid poly (vinyl chloride) (PVC) profile extrusions, Nat. Bur. Stand. (U.S.), Voluntary Prod. Stand. 26-70, 7 pages (Oct. 1970) 10 cents, SD Catalog No. C13.20/2:26-70.

Straty, G. C., Prydz, R., Fluorine compatible apparatus for accurate PVT measurements, Rev. Sci. Instr. 41, No. 8, 1223-1227 (August 1970).

Thomas, A. M., Further study of vacuum vane gauge criteria: Effects of port-vane geometry, J. Vacuum Sci. Technol. 7, No. 4, 501-503 (July-August - 1970).

Weidman, M. P., Campbell, E., A method for designing multi-screw waveguide tuners, Nat. Bur. Stand. (U.S.), Tech. Note 393, 20 pages (Oct. 1970) 30 cents, SD Catalog No. C13.46:393.

White, H., Editor, Vocabulary for Information Processing, Nat. Bur. Stand. (U.S.), Fed. Info. Process. Stand. Publ. (FIPS Pub.) 11, 4 pages (1970) 15 cents, SD Catalog No. C13.52:11.

Yakowitz, H., Some uses of color in metallography, Am. Soc. Testing Mater., Spec. Publ. 480, pp. 49-66 (1970).

Yokel, F. Y., Somes, N. F., Proposed revision of ACI 318-63 Building Code Requirements for Reinforced Concrete, J. Am. Concrete Inst. 67, No. 9, 723-725 (September 1970).

Young, J. P., Lamb, V. A., Reid, G. I., Berkeley, J. F., Ng, W., Electroplated coatings on maraging steel for retarding catalytic decomposition of hydrazine rocket fuel, Plating 57, No. 9, 921926 (September 1970).

Younglove, B. A., Straty, G. C., A capacitor for accurate wide range dielectric constant measurements on compressed fluids, Rev. Sci. Instr. 41, No. 7, 1087-1089 (July 1970).

Zimmerman, J. E., Low cost sensors with millikelvin temperatures and other virtues (Summary), Proc. Conf. Precision Electromagnetic Measurements, National Bureau of Standards, Boulder, Colorado, June 2-5, 1970, CPEM Digest, p. 1 (1970).

*Publications for which a price is indicated are available by purchase from the Superintendent of Documents, U.S. Government Printing Office, Washington, D.C. 20402 (foreign postage, one-fourth additional). The NBS non-periodical series are also available from the National Technical Information Service (NTIS), Springfield, Va. 22151, which was formerly the Clearinghouse. Reprints from outside journals and the NBS Journal of Research may often be obtained directly from the authors. 